\title{
Stability for quasi-periodically perturbed Hill's equations
}

\author{
Guido Gentile ${ }^{\dagger}$, Daniel A. Cortez* and João C. A. Barata* \\ ${ }^{\dagger}$ Dipartimento di Matematica, Università di Roma Tre, Roma, I-00146, Italy. \\ E-mail: gentile@mat.unirom3.it \\ *Instituto de Física, Universidade de São Paulo, Caixa Postal 66 318, \\ São Paulo, 05315970 SP, Brasil. \\ E-mails: dacortez, jbarata@fma.if.usp.br
}

\begin{abstract}
We consider a perturbed Hill's equation of the form $\ddot{\phi}+\left(p_{0}(t)+\varepsilon p_{1}(t)\right) \phi=0$, where $p_{0}$ is real analytic and periodic, $p_{1}$ is real analytic and quasi-periodic and $\varepsilon \in \mathbb{R}$ is "small". Assuming Diophantine conditions on the frequencies of the decoupled system, i.e. the frequencies of the external potentials $p_{0}$ and $p_{1}$ and the proper frequency of the unperturbed $(\varepsilon=0)$ Hill's equation, but without making non-degeneracy assumptions on the perturbing potential $p_{1}$, we prove that quasi-periodic solutions of the unperturbed equation can be continued into quasi-periodic solutions if $\varepsilon$ lies in a Cantor set of relatively large measure in $\left[-\varepsilon_{0}, \varepsilon_{0}\right] \subset \mathbb{R}$, where $\varepsilon_{0}$ is small enough. Our method is based on a resummation procedure of a formal Lindstedt series obtained as a solution of a generalized Riccati equation associated to Hill's problem.
\end{abstract}

\section{Introduction}

In the present work we will consider the one-dimensional Hill's equation (for a standard reference, see [22]) with a quasi-periodic perturbation

$$
\ddot{\phi}+\left(p_{0}(t)+\varepsilon p_{1}(t)\right) \phi=0,
$$

where $p_{0}$ and $p_{1}$ are two real analytic functions, the first periodic with frequency $\omega_{0}$ and the latter quasiperiodic with frequency vector $\underline{\omega}_{1} \in \mathbb{R}^{A}$, for an integer $A \geq 1$ (for notational details see Section 1.1). No further assumption is made on the equation, besides requiring that the real parameter $\varepsilon$ is small and that the unperturbed equation (i.e. for $\varepsilon \equiv 0$ ) has a fundamental system of real quasi-periodic solutions.

For $p_{0}$ constant such an equation has been extensively studied, also in connection with the spectrum of the corresponding Schrödinger equation $\ddot{\phi}+\varepsilon V\left(\underline{\omega}_{1} t\right) \phi=E \phi$, with $V$ analytic and periodic in its arguments; see for instance Refs. 10, 25, 11, 18, 26, 23. We also mention the recent Ref. 4] and also [5, where some properties of the gaps and of the instability tongues have been investigated. Different perturbations of Hill's equation, with a $L^{1}$ perturbing potential, have been considered for instance in Refs. 24, 27, 28, 17].

We are interested in the problem of studying conservation of quasi-periodic motions for $\varepsilon$ different from zero but small enough. Of course, equation (1.1) can be considered as arising from an autonomous Hamiltonian system with $d=A+2$ degrees of freedom, described by the Hamiltonian

$$
H=\Omega_{0} A+\omega_{0} A_{0}+\underline{\omega}_{1} \cdot \underline{A}_{1}+\varepsilon p_{1}\left(\underline{\alpha}_{1}\right) f\left(A, A_{0}, \alpha, \alpha_{0}\right),
$$


where $\left(A, A_{0}, \underline{A}_{1}, \alpha, \alpha_{0}, \underline{\alpha}_{1}\right) \in \mathbb{R} \times \mathbb{R} \times \mathbb{R}^{A} \times \mathbb{T} \times \mathbb{T} \times \mathbb{T}^{A}$ are action-angle variables, and $f$ and $\Omega_{0}$ depend on the periodic potential $p_{0}$. For instance if $p_{0}$ is a constant, say $p_{0}=1$, then the variables $\left(A_{0}, \alpha_{0}\right)$ disappear, $\Omega_{0}=1$ and $f(A, \alpha)=2 A \cos ^{2} \alpha$. In general the change of variables leading to (1.2) is slightly more complicated, but it can be easily worked out; we refer for instance to Refs. 8, 9]. Also in such a case the function $f$ is linear in the action variables. Hence systems like (1.2) are not typical in KAM theory, because the perturbation does not remove isochrony. What one usually does is to study the behavior of the solutions, in particular to understand if they are bounded (quasi-periodic) or unbounded (linearly or exponentially growing), when varying the parameters characterizing the external potential. In the case of the Schrödinger equation this can be done for a fixed potential, by varying the energy, which represents an extra free parameter, and information can be obtained about the spectrum. In Ref. 9] this is done for bounded solutions, so that conditions on $E$ are obtained characterizing the spectrum of the corresponding Schrödinger operator.

Here we are interested in the case in which the potential is fixed, and the parameters of $p_{0}$ are such that the fundamental solutions of the corresponding Hill's equation $\ddot{\phi}+p_{0}(t) \phi=0$ are quasi-periodic (this means that we are inside the stability regions). Hence for $\varepsilon=0$ we have $d=A+2$ fundamental frequencies $\underline{\omega}_{1}, \omega_{0}, \Omega_{0}$, where $\Omega_{0}$ is the proper frequency of the unperturbed Hill's equation. Then we want to study if the solutions remain quasi-periodic when the perturbation is switched on. Even when this occurs, one expects that the proper frequency of the system is changed as an effect of the perturbation. Since the system is in fact a perturbation of an isochronous one, and we have no free parameter to adjust, either the proper frequency is changed to some perturbation order or it is never changed (if disposing of the extra parameter $E$ the frequency changes to first order up to a zero-measure set). But to follow all the possibilities requires some careful analysis, which one can avoid by assuming some non-degeneracy condition on the perturbation in order to control the change of the frequencies. On the contrary we do not want to impose any condition on the perturbation.

Degeneracy problems of this kind are known to be not easy to handle. An example is given by Herman's conjecture in the case in which one has a system of $N$ harmonic oscillators where no assumption is made on the coupling terms of order higher than two: in such a case the conservation of a large measure of invariant tori has been be proved only for $N=2$ [16]. We can mention also Cheng's results on the conservation of lower $(N-1)$-dimensional tori for systems with $N$ degrees of freedom [6. 7.

To come back to our problem, we fix the unperturbed torus and study for which values of $\varepsilon$ (small enough) such a torus is conserved. In particular we are interested in the dependence on $\varepsilon$ of the conserved torus: we shall find that the torus will be defined for $\varepsilon$ in a Cantor set of large relative measure, and for such values of $\varepsilon$ the system turns out to be reducible. We shall see also that one can give a meaning to the perturbation series, through a suitable resummation, in an analogous way to what was done in similar contexts in Refs. 13, 12, 14.

We do not study directly the equation (1.1). Rather, we shall write $\phi$ in terms of a suitable function $u$, for which a very simple-looking equation can be derived. Indeed by setting

$$
\phi_{0}(t)=\text { const. } \exp \left(i \int_{0}^{t} g_{0}\left(t^{\prime}\right) \mathrm{d} t^{\prime}\right), \quad Q(t)=\exp \left(-2 i \int_{0}^{t} g_{0}\left(t^{\prime}\right) \mathrm{d} t^{\prime}\right),
$$

where $\phi_{0}$ is a quasi-periodic solution of (1.1) for $\varepsilon=0$, with rotation vector $\left(\omega_{0}, \Omega_{0}\right)$, where the proper frequency $\Omega_{0}$ is the average of $g_{0}$, and defining

$$
\phi(t)=\phi_{0}(t) \exp \left(i \int_{0}^{t} g\left(t^{\prime}\right) \mathrm{d} t^{\prime}\right), \quad g(t)=i \varepsilon Q(t) u(t),
$$

one finds that $u$ has to solve the equation (see Section 2.2 for details)

$$
\dot{u}=R+\varepsilon Q u^{2}, \quad R=p_{1} Q^{-1},
$$

which is an ordinary differential equation which could be of interest by its own. 
The advantage of this procedure is that we can look for a solution of (1.4) with the same rotation vector $\boldsymbol{\omega}=\left(\underline{\omega}_{1}, \omega_{0}, \Omega_{0}\right)$ of the unperturbed system, something which cannot be done for the full unperturbed system, as the proper frequency $\Omega_{0}$ is expected to change (as usually happens when perturbing an isochronous system).

That such a solution $u(t)$ exists can be shown, and this is the core of the paper, provided one assumes, besides an obvious Diophantine condition on $\boldsymbol{\omega}$, that $\varepsilon$ is small enough, say $|\varepsilon| \leq \varepsilon_{0}$, and belongs to a suitable Cantor set $\mathcal{E}$ of large relative measure in $\left[-\varepsilon_{0}, \varepsilon_{0}\right]$. By the latter we mean that one has $\lim _{\varepsilon \rightarrow 0^{+}} \operatorname{meas}(\mathcal{E} \cap[-\varepsilon, \varepsilon]) / 2 \varepsilon=1$, with meas denoting Lebesgue measure.

To recover the solution $\phi(t)$ we have to express it in terms of $u$. By using the relations given in (1.3) one realizes that, first, the solution could be unbounded (if the imaginary part of the average $\langle g\rangle$ of $g$ did not vanish), and, second, even if this did not occur, an extra frequency $\Omega_{\varepsilon}=\Omega_{0}+\langle g\rangle$ would appear in addition to the $d$ frequencies already characterizing the model, which would sound strange. But one can check that both problems are spurious, as $\langle g\rangle$ turns out to be real and dependence on time of the function $\phi(t)$, which, in principle, could be through the variables $\underline{\omega}_{1} t, \omega_{0} t, \Omega_{0} t, \Omega_{\varepsilon} t$ (by construction), is indeed only through the variables $\underline{\omega}_{1} t, \omega_{0} t, \Omega_{\varepsilon} t$, as formally noticed in the case treated in [1]. In other words, the dependence on $\Omega_{0} t$ disappears, and this means that the maximal torus, which in absence of perturbation has rotation vector $\left(\underline{\omega}_{1}, \omega_{0}, \Omega_{0}\right)$, can be continued for $\varepsilon \in \mathcal{E}$, and the last component of the rotation vector is changed into an $\varepsilon$-dependent quantity $\Omega_{\varepsilon}$ (that the other components cannot change is obvious by the form of the equations of motion). Hence the solution of (1.4) provides directly a perturbation expansion for the correction of the proper frequency of the system: indeed $\Omega_{\varepsilon}-\Omega_{0}=\langle g\rangle$, and $g$ is expressed in terms of the solution $u$.

We can now state our results in the following theorem.

Theorem 1.1 Let $p_{0}: \mathbb{R} \rightarrow \mathbb{R}$ be real analytic and periodic with frequency $\omega_{0}$ and such that the fundamental solutions of the corresponding Hill's equation $\ddot{\phi}+p_{0}(t) \phi=0$ are quasi-periodic with a proper frequency $\Omega_{0} \in \mathbb{R}$. Let $p_{1}: \mathbb{R} \rightarrow \mathbb{R}$ be real analytic and quasi-periodic with frequency vector $\underline{\omega}_{1} \in \mathbb{R}^{A}$ for some $A \geq 1$. Define $\boldsymbol{\omega}:=\left(\underline{\omega}_{1}, \omega_{0}, \Omega_{0}\right) \in \mathbb{R}^{d}$ with $d=A+2$ and assume that $\underline{m} \cdot \underline{\omega}_{1}+n \omega_{0}+2 \Omega_{0} \neq 0, \forall(\underline{m}, n) \in \mathbb{Z}^{A+1}$ and, moreover

$$
|\boldsymbol{\omega} \cdot \boldsymbol{\nu}| \geq \frac{C_{0}}{|\boldsymbol{\nu}|^{\tau}}, \quad \forall \boldsymbol{\nu} \in \mathbb{Z}^{d} \backslash\{\mathbf{0}\},
$$

for two fixed positive constants $C_{0}>0$ and $\tau>d-1$ (Diophantine conditions). Then, there exists $\varepsilon_{0}>0$ small enough and a Cantor set $\mathcal{E} \subset\left[-\varepsilon_{0}, \varepsilon_{0}\right]$ of large relative measure in $\left[-\varepsilon_{0}, \varepsilon_{0}\right]$ such that, for all $\varepsilon \in \mathcal{E}$, 1.4) admits a quasi-periodic solution of the form

$$
\bar{u}(t)=U(\boldsymbol{\omega} t ; \varepsilon)=\sum_{\boldsymbol{\nu} \in \mathbb{Z}^{d}} \tilde{U}_{\boldsymbol{\nu}}(\varepsilon) e^{i \boldsymbol{\omega} \cdot \boldsymbol{\nu} t},
$$

where the sum above is absolutely and uniformly convergent for all $t \in \mathbb{R}$ and all $\varepsilon \in \mathcal{E}$. Moreover, for all $\varepsilon \in \mathcal{E}$, the system (1.1) is reducible and it has a quasi-periodic solution of the form

$$
\phi(t)=\Phi\left(\Omega_{\varepsilon} t, \underline{\omega}_{1} t, \omega_{0} t ; \varepsilon\right)=e^{i \Omega_{\varepsilon} t}\left(\sum_{(\underline{m}, n) \in \mathbb{Z}^{A+1}} \tilde{\Phi}_{\underline{m}, n}(\varepsilon) e^{i\left(\underline{m} \cdot \underline{\omega}_{1}+n \omega_{0}\right) t}\right),
$$

where, by denoting with $\langle\cdot\rangle$ the average of a quasi-periodic function (that is the constant term in its Fourier expansion), one has $\Omega_{\varepsilon}:=\Omega_{0}+\langle g\rangle=\Omega_{0}+i \varepsilon\langle Q u\rangle$ is real, and the sum above is absolutely and uniformly convergent for all $t \in \mathbb{R}$ and all $\varepsilon \in \mathcal{E}$. Finally, if $\langle g\rangle=0$ then $\mathcal{E}=\left[-\varepsilon_{0}, \varepsilon_{0}\right]$ and $\Omega_{\varepsilon}$ reduces to $\Omega_{0}$.

In particular the proof of the result will imply that the equation is reducible for $\varepsilon \in \mathcal{E}$. It would be interesting to study what happens for $\varepsilon$ outside the set $\mathcal{E}$ (cf. the results proved for the case of the Schrödinger equation with $p_{0}=0$ and other related models [11, 20, 21]). 
The rest of this paper is devoted to the proof of the above theorem.

We organize this work as follows: in Section 2 we motivate and discuss the Ansatz used to solve (1.1) and introduce the tree representation of the perturbative coefficients obtained, which is the basis for the forthcoming analysis. Section 3 is devoted to the solution of the "zero mode" problem, which is essential for constructing a consistent quasi-periodic solution for (1.4). Section 4 shows that our naive perturbative solution is merely formal, i.e. not convergent as a power series in $\varepsilon$. This is related to small divisors problems. Next, Section 5 brings the core idea of this paper: the renormalization of the formal solution. This process is implemented through a multiscale decomposition of propagators and a suitable resummation technique. As described in Theorem 1.1 the result is a convergent quasi-periodic solution for (1.4), well defined in a Cantor set $\mathcal{E}$ of relatively large measure in $\left[-\varepsilon_{0}, \varepsilon_{0}\right]$. Section [6] is devoted to the proof of some technical lemmas which are related to estimates on the so called "selfenergy values". This lemmas are crucial in the proof of Theorem 1.1] which is essentially performed in Section [7] where convergence of the renormalized expansion is shown. Next, in Section 8 we provide estimates on the measure of the set $\mathcal{E}$ where the renormalized solution exists. It is shown that $\mathcal{E}$ is of relatively large measure in a compact set $\left[-\varepsilon_{0}, \varepsilon_{0}\right]$. Finally, Section 9 completes the proof of Theorem 1.1 by analyzing properties of the renormalized expansion. Section 10 closes the paper by discussing the rather trivial situation where we cannot fix the zero modes as in Section 3 This is the situation where the proper frequency of the unperturbed Hill's equation in unchanged when the perturbation is switched on, i.e. $\Omega_{\varepsilon}=\Omega_{0}$.

\subsection{Basic notations}

In this paper $\mathbb{N}$ will denote the set of positive integers, $\mathbb{Z}$ the set of all integers and $\mathbb{R}$ the set of real numbers. Note that $0 \notin \mathbb{N}$. For any $n \in \mathbb{N}, \mathbb{Z}^{n}$ (or $\mathbb{R}^{n}$ ) is the Cartesian product of $\mathbb{Z}$ (or $\mathbb{R}$ ) $n$ times. The set $\mathbb{T}$ denotes the one-dimensional torus, i.e. $\mathbb{T}=\mathbb{R} / 2 \pi \mathbb{Z}$. $\mathbb{T}^{n}$ is the $n$-dimensional torus.

Vectors in $\mathbb{Z}^{n}$ (or $\mathbb{R}^{n}$ ) will be denoted either by boldface or underline characters. Boldface characters will be used to denote vector in a certain dimension $d$, i.e. $\boldsymbol{\omega} \in \mathbb{R}^{d}, \boldsymbol{\nu} \in \mathbb{Z}^{d}$. Underline characters will be used to denote vector in a certain dimension $A<d$, i.e. $\underline{\omega}_{1} \in \mathbb{R}^{A}, \underline{m} \in \mathbb{Z}^{A}$.

For any $n \in \mathbb{N}, \mathbb{Z}_{*}^{n}$ is defined as $\mathbb{Z}^{n} \backslash\{\mathbf{0}\}$, i.e. $\mathbb{Z}_{*}^{n}$ is $\mathbb{Z}^{n}$ with the exception of the zero. The same applies to $\mathbb{R}^{n}$.

The scalar product in $\mathbb{R}^{n}$ will be denoted as usual by a dot: $\mathbf{v} \cdot \mathbf{w}:=v_{1} w_{1}+\cdots+v_{n} w_{n}$, for $\mathbf{v}, \mathbf{w} \in \mathbb{R}^{n}$. The $\ell^{1}$-norm of a vector $\mathbf{v}=\left(v_{1}, \ldots, v_{n}\right) \in \mathbb{R}^{n}$ is $|\mathbf{v}|:=\left|v_{1}\right|+\cdots+\left|v_{n}\right|$, where in the r.h.s. $|\cdot|$ denotes the usual absolute value in $\mathbb{R}$ (or $\mathbb{C}$ ). The complex conjugate of $z \in \mathbb{C}$ will be denoted by $z^{*}$.

Given a periodic or, more generally, a quasi-periodic function $f$ we denote by $\langle f\rangle$ the average of $f$,

$$
\langle f\rangle:=\lim _{T \rightarrow \infty} \frac{1}{2 T} \int_{-T}^{T} \mathrm{~d} t f(t)=f_{\mathbf{0}},
$$

where $f_{\mathbf{0}}$ is the constant term of the Fourier expansion of $f$ [19].

The symbol $\square$ will be used at the end of the statement of a theorem, lemma or proposition and will be used at the end of a proof.

\section{Perturbative analysis}

In this Section we will begin our perturbative analysis. We start from a given complex quasi-periodic solution for the unperturbed version of (1.1), i.e. for $\varepsilon=0$, and search for a perturbative solution for the full equation that formally tends to this unperturbed solution as $\varepsilon \rightarrow 0$. For this, we apply an exponential Ansatz, whose geometrical motivation we briefly discuss below, leading to a generalized Riccati equation (equation (2.9), ahead). In the core of this paper we prove that this generalized 
Riccati equation admits a quasi-periodic solution under suitable conditions on the frequencies and on the coupling parameter $\varepsilon$ and, as we prove below, this implies quasi-periodicity of the perturbed solution of (1.1). In Section 2.3 we present a formal tree expansion for the solution of (2.9) that will be the starting point of our renormalization analysis.

However, as we shall see, boundness on the solutions of (2.9) will automatically imply stability on the associate solutions of Hill's equation. This will become more clear with Proposition 2.3

\subsection{Unperturbed equation}

The following elementary result presents some basic properties of complex quasi-periodic solutions of the unperturbed Hill's equation that partially motivates the approach of Section 2.2

Proposition 2.1 Let $p_{0}: \mathbb{R} \rightarrow \mathbb{R}$ be an analytic periodic function with period $T_{0}=2 \pi / \omega_{0}$, such that the equation

$$
\ddot{\phi}(t)+p_{0}(t) \phi(t)=0
$$

has two non-trivial, real, analytic, quasi-periodic and independent solutions $\phi_{a}$ and $\phi_{b}$. Then, the complex quasi-periodic solution $\phi_{0}(t)=\phi_{a}(t)+i \phi_{b}(t)$ can be expressed in the form

$$
\phi_{0}(t)=\exp \left(i \Omega_{0} t+i \psi_{0}(t)\right)
$$

where $\Omega_{0} \in \mathbb{R}$ and $\psi_{0}: \mathbb{R} \rightarrow \mathbb{C}$ is an analytic periodic function with frequency $\omega_{0}$.

Proof. Since the Wronskian $W(t)=\phi_{a}(t) \dot{\phi}_{b}(t)-\phi_{b}(t) \dot{\phi}_{a}(t)$ is a non-vanishing constant, $W(t)=W_{0} \neq$ $0, \forall t \in \mathbb{R}$, one has

$$
\left|W_{0}\right| \leq\left|\phi_{a}(t)\right|\left|\dot{\phi}_{b}(t)\right|+\left|\phi_{b}(t)\right|\left|\dot{\phi}_{a}(t)\right| \leq D\left(\left|\phi_{a}(t)\right|+\left|\phi_{b}(t)\right|\right),
$$

where $D:=\max \left\{\sup _{t \in \mathbb{R}}\left|\dot{\phi}_{a}(t)\right|, \sup _{t \in \mathbb{R}}\left|\dot{\phi}_{b}(t)\right|\right\}<\infty$, because $\dot{\phi}_{a}$ and $\dot{\phi}_{b}$ are both, by hypothesis, quasi-periodic. Let $\phi_{0}:=\phi_{a}+i \phi_{b}$. By the equivalence of the $\ell^{1}$ and $\ell^{2}$ norms, there exists a constant $C>0$ such that

$$
\left|\phi_{0}(t)\right|=\sqrt{\left|\phi_{a}(t)\right|^{2}+\left|\phi_{b}(t)\right|^{2}} \geq C\left(\left|\phi_{a}(t)\right|+\left|\phi_{b}(t)\right|\right) \geq \frac{C\left|W_{0}\right|}{D}, \quad \forall t .
$$

This tells us that the quasi-periodic complex function $\phi_{0}$ remains outside of a neighborhood of the origin for all times. Under these circumstances, a theorem of H. Bohr 3 , implies that we can write

$$
\phi_{0}(t)=\exp \left(i \Omega_{0} t+i \psi_{0}(t)\right)
$$

where $\Omega_{0} \in \mathbb{R}$ and $\psi_{0}(t): \mathbb{R} \rightarrow \mathbb{C}$ is almost periodic. Floquet's theorem guarantees that $\psi_{0}$ is periodic with the same frequency of $p_{0}$.

We clearly see from (2.3) that $\Omega_{0}$ is the rotation number of $\phi_{0}$.

Since $\phi_{0}^{*}$ is also a solution of (2.1) (because (2.1) is real), the most general (complex) solution is

$$
A_{1} \exp \left(+i \Omega_{0} t+i \psi_{0}(t)\right)+A_{2} \exp \left(-i \Omega_{0} t-i \psi_{0}(t)^{*}\right),
$$

with $A_{1}, A_{2} \in \mathbb{C}$.

Defining the periodic function $g_{0}(t):=\dot{\psi}_{0}(t)+\Omega_{0}$, we can write

$$
\phi_{0}(t)=\exp \left(i \int_{0}^{t} g_{0}\left(t^{\prime}\right) \mathrm{d} t^{\prime}\right) e^{i \psi_{0}(0)} .
$$

Since $\left\langle\dot{\psi}_{0}\right\rangle=0$, we have $\Omega_{0}=\left\langle g_{0}\right\rangle$. 


\subsection{Perturbed equation and the exponential Ansatz}

As we mentioned, the representation (2.5) is possible because (2.2) tells us that the quasi-periodic complex function $\phi_{0}$ runs outside of a neighborhood of the origin for all times. It is tempting to presume that this sort of stability property is preserved when the perturbation is switched on and that the periodic function $g_{0}$ is replaced by a quasi-periodic one in the form $g_{0}+g$, where $g$ vanishes when $\varepsilon \rightarrow 0$. This is the motivation for the steps that follow.

Let us now consider the perturbed equation (1.1) with $p_{1}: \mathbb{R} \rightarrow \mathbb{R}$ analytic and quasi-periodic, with frequencies in the set $\left\{\underline{m} \cdot \underline{\omega}_{1}, \underline{m} \in \mathbb{Z}^{A}\right\}$ for some $A \geq 1$. The motivations presented above (see also [1) lead us to search for a solution of (1.1) with the following form

$$
\phi(t)=\phi_{0}(t) \exp \left(i \int_{0}^{t} g\left(t^{\prime}\right) \mathrm{d} t^{\prime}\right)=\exp \left(i \int_{0}^{t}\left[g_{0}\left(t^{\prime}\right)+g\left(t^{\prime}\right)\right] \mathrm{d} t^{\prime}\right) e^{i \psi_{0}(0)},
$$

with $g$ vanishing identically for $\varepsilon=0$. It is easily verifiable that $g$ must satisfy the following generalized Riccati equation:

$$
\frac{\mathrm{d}}{\mathrm{d} t}\left(g \phi_{0}^{2}\right)+i\left(g \phi_{0}\right)^{2}-i \varepsilon p_{1} \phi_{0}^{2}=0
$$

or, in another form,

$$
\dot{g}+i g^{2}+2 i g_{0} g-i \varepsilon p_{1}=0
$$

Remark 2.2 Of course in this way we are considering a solution which reduces to the first function in 2.4) for $\varepsilon=0$. In the following we could also consider solutions continuing for $\varepsilon \neq 0$ the second function in 2.4, and the analysis would be the same.

The idea now is to search for a quasi-periodic solution $g$ for the above equation. In this case,

$$
\phi(t)=\exp \left(i \Omega_{\varepsilon} t+i \psi_{\varepsilon}(t)\right)
$$

where

$$
\Omega_{\varepsilon}:=\Omega_{0}+\langle g\rangle \quad \text { and } \quad \psi_{\varepsilon}(t):=\psi_{0}(t)+\int_{0}^{t}\left(g\left(t^{\prime}\right)-\langle g\rangle\right) \mathrm{d} t^{\prime} .
$$

Note that, if such a $g$ exists, $\psi_{\varepsilon}$ would be also quasi-periodic. However, in order to assure that $\phi$ is quasi-periodic we have to show that $\Omega_{\varepsilon}$ is a real number, which is the case iff $\langle g\rangle \in \mathbb{R}$. This is established by the following proposition that shows that if $g$ is quasi-periodic, then $\phi$ is automatically stable, i.e. the Lyapunov exponent $\operatorname{Im}\left(\Omega_{\varepsilon}\right)$ vanishes.

Proposition 2.3 Let us assume that 2.7) has a quasi-periodic solution $g$. Then the average of $g$ is real, that is $\langle g\rangle \in \mathbb{R}$.

Proof. Write $g_{0}=x_{0}+i y_{0}$ and $g=x+i y$. Note that $\left\langle g_{0}\right\rangle=\Omega_{0} \in \mathbb{R}$, hence $\left\langle y_{0}\right\rangle=0$. One has $i \dot{g}_{0}-g_{0}^{2}+p_{0}=0$, whose imaginary part gives $\dot{x}_{0}=2 x_{0} y_{0}$. Moreover, one has $\dot{g}+i g^{2}+2 i g_{0} g-i \varepsilon p_{1}=0$ (equation (2.8) ), whose real part is $\dot{x}-2 x y-2 x y_{0}-2 y x_{0}=0$. Combining the two equations we obtain $\dot{x}-2 x y-2 x_{0} y-2 x y_{0}+\left(-2 x_{0} y_{0}+\dot{x}_{0}\right)=0$, hence $\dot{x}+\dot{x}_{0}-2\left(y+y_{0}\right)\left(x+x_{0}\right)=0$.

By defining $z=x+x_{0}$ the above equation becomes $\dot{z}=f(t) z$, where the function $f(t)=2(y(t)+$ $\left.y_{0}(t)\right)$ is bounded (and quasi-periodic), hence, by explicit integration,

$$
z(t)=\exp \left(2 \int_{0}^{t}\left[y_{0}\left(t^{\prime}\right)+y\left(t^{\prime}\right)\right] \mathrm{d} t^{\prime}\right) z(0)
$$

where $z(0)=x_{0}(0)+x(0) \neq 0$ (if $z(0)=0$ then $z(t) \equiv 0$ for all $t$, hence $x(t)=-x_{0}(t)$ for all $t$, which requires $x_{0}(t)=x(t) \equiv 0$ for all $t$, and this is not possible as $\left\langle x_{0}\right\rangle=\Omega_{0} \neq 0$, so that $x_{0}(t)$ cannot vanish 
identically). On the other hand $z(t)$ has to be a bounded quasi-periodic function, and this requires $\left\langle y_{0}+y\right\rangle=0$, so that one has $\langle y\rangle=0$.

Therefore, we can establish that $\phi(t)$ given in (2.6) is quasi-periodic provided we find a quasiperiodic $g$. Further remarks on properties of $\phi$ will be discussed in Section 9

A slightly simpler version of the generalized Riccati equation (2.7) above was studied in 13 by a tree expansion method (see, e.g., [15] and references therein). So, the idea now is to try to write the same expansion of 13 for a solution of (2.7) and to adapt its analysis (and results) to the context of the problem posed here.

First of all, let us rewrite the Riccati equation (2.7) as in 13. Since $\phi_{0} \neq 0$ for all $t \in \mathbb{R}$, we define $u(t)$ by

$$
g(t)=i \varepsilon Q(t) u(t)
$$

where

$$
Q(t):=\exp \left(-2 i \int_{0}^{t} g_{0}\left(t^{\prime}\right) \mathrm{d} t^{\prime}\right)=\left(\phi_{0}(t)\right)^{-2},
$$

which, by (2.3), is also quasi-periodic. We also define,

$$
R(t):=p_{1}(t) Q(t)^{-1}=p_{1}(t) \phi_{0}^{2}(t)=p_{1}(t) \exp \left(2 i \int_{0}^{t} g_{0}\left(t^{\prime}\right) \mathrm{d} t^{\prime}\right) .
$$

With the above definitions one trivially checks from (2.7) that

$$
\dot{u}=R+\varepsilon Q u^{2},
$$

which is very similar to the equation studied in [13].

\subsection{Tree expansion}

Now we pass to the perturbative expansions and a graphic representation that will conduct our analysis. As a first attempt (and also just to introduce notations) we search for a solution of (2.9) as a power series in $\varepsilon$ :

$$
u(t)=\sum_{k=0}^{\infty} \varepsilon^{k} u^{(k)}(t) .
$$

Note that, in principle, $u$ does not vanish identically for $\varepsilon=0$, but $g$ does, since $g \sim \varepsilon u$. By inserting the above Ansatz into equation (2.9), we arrive at

$$
\begin{aligned}
& \dot{u}^{(0)}=R, \\
& \dot{u}^{(k)}=Q \sum_{k_{1}+k_{2}=k-1} u^{\left(k_{1}\right)} u^{\left(k_{2}\right)}, \quad \forall k \geq 1 .
\end{aligned}
$$

Since we search for a quasi-periodic solution $u$ of (2.9), it is natural to introduce the following Fourier decomposition:

$$
u^{(k)}(t)=\sum_{\boldsymbol{\nu} \in \mathbb{Z}^{d}} u_{\boldsymbol{\nu}}^{(k)} e^{i \boldsymbol{\nu} \cdot \boldsymbol{\omega} t},
$$

for some $d \geq 1$ to be conveniently fixed later. Note that with the above decomposition, we have

$$
u(t)=\sum_{k=0}^{\infty} \varepsilon^{k} \sum_{\boldsymbol{\nu} \in \mathbb{Z}^{d}} u_{\boldsymbol{\nu}}^{(k)} e^{i \boldsymbol{\nu} \cdot \boldsymbol{\omega} t} .
$$


Our goal now is to find a graphical representation in terms of trees for the Fourier coefficients $u_{\nu}^{(k)}$, as in 13 .

We now proceed and write the Fourier decomposition of the functions $p_{0}, p_{1}, \phi_{0}, Q$ and $R$. Since $p_{0}$ is assumed periodic (with period $T_{0}=2 \pi / \omega_{0}$ ), we simply have

$$
p_{0}(t)=\sum_{n \in \mathbb{Z}} P_{n}^{(0)} e^{i n \omega_{0} t} .
$$

The function $p_{1}$ is assumed quasi-periodic with spectrum of frequencies contained in the set $\{\underline{m}$. $\left.\underline{\omega}_{1}, \underline{m} \in \mathbb{Z}^{A}\right\}$. Hence,

$$
p_{1}(t)=\sum_{\underline{m} \in \mathbb{Z}^{A}} P_{\underline{m}}^{(1)} e^{i \underline{m} \cdot \underline{\omega}_{1} t} .
$$

We write the Fourier decompositions of $\phi_{0}^{2}$ and $\phi_{0}^{-2}$ as follows:

$$
\left(\phi_{0}(t)\right)^{2}=\sum_{n \in \mathbb{Z}} \mathcal{F}_{n}^{(2)} e^{i\left(n \omega_{0}+2 \Omega_{0}\right) t}, \quad\left(\phi_{0}(t)\right)^{-2}=\sum_{n \in \mathbb{Z}} \mathcal{F}_{n}^{(-2)} e^{i\left(n \omega_{0}-2 \Omega_{0}\right) t} .
$$

Therefore, the Fourier decomposition of $R$ is

$$
R(t)=\sum_{\underline{m} \in \mathbb{Z}^{A}} P_{\underline{m}}^{(1)} e^{i \underline{m} \cdot \underline{\omega}_{1} t} \sum_{n \in \mathbb{Z}} \mathcal{F}_{n}^{(2)} e^{i\left(n \omega_{0}+2 \Omega_{0}\right) t}=\sum_{\boldsymbol{\nu} \in \mathbb{Z}^{d}} R_{\boldsymbol{\nu}} e^{i \boldsymbol{\nu} \cdot \boldsymbol{\omega} t},
$$

where

$$
\boldsymbol{\nu}:=\left(\underline{m}, n_{1}, n_{2}\right), \quad d:=A+2, \quad \boldsymbol{\omega}:=\left(\underline{\omega}_{1}, \omega_{0}, \Omega_{0}\right)
$$

and

$$
R_{\nu}:=P_{\underline{m}}^{(1)} \mathcal{F}_{n_{1}}^{(2)} \delta_{n_{2}, 2} .
$$

With this notation, the Fourier decomposition of $Q$ is as follows:

$$
Q(t)=\sum_{n \in \mathbb{Z}} \mathcal{F}_{n}^{(-2)} e^{i\left(n \omega_{0}-2 \Omega_{0}\right) t}=\sum_{\boldsymbol{\nu} \in \mathbb{Z}^{d}} Q_{\boldsymbol{\nu}} e^{i \boldsymbol{\nu} \cdot \boldsymbol{\omega} t},
$$

where $\boldsymbol{\nu}, d$ and $\boldsymbol{\omega}$ are as (2.13) and

$$
Q_{\nu}:=\delta_{\underline{m}, \underline{0}} \mathcal{F}_{n_{1}}^{(-2)} \delta_{n_{2},-2} .
$$

Remark 2.4 We assume the following non-resonant condition on the frequency vector $\boldsymbol{\omega}$ :

$$
\underline{m} \cdot \underline{\omega}_{1}+n \omega_{0}+2 \Omega_{0} \neq 0 \quad \forall(\underline{m}, n) \in \mathbb{Z}^{A+1} .
$$

We also impose a Diophantine condition on $\boldsymbol{\omega}$, namely:

$$
|\boldsymbol{\omega} \cdot \boldsymbol{\nu}| \geq \frac{C_{0}}{|\boldsymbol{\nu}|^{\tau}} \quad \forall \boldsymbol{\nu} \in \mathbb{Z}_{*}^{d},
$$

with $\mathbb{Z}_{*}^{d}:=\mathbb{Z}^{d} \backslash\{\mathbf{0}\}$, for two fixed positive constants $C_{0}$ and $\tau>d-1$.

Remark 2.5 By the analyticity assumption on $p_{0}$ and $p_{1}$ one obtain the following decay for the Fourier coefficients of $Q$ and $R$ :

$$
\left|R_{\boldsymbol{\nu}}\right| \leq \mathcal{Q} e^{-\kappa|\boldsymbol{\nu}|}, \quad\left|Q_{\boldsymbol{\nu}}\right| \leq \mathcal{Q} e^{-\kappa|\boldsymbol{\nu}|},
$$

for some positive constants $\mathcal{Q}$ and $\kappa$. This will be essential in our forthcoming analysis. 
We now proceed and insert the decomposition (2.11) into (2.10). The result is the following recursive relations for the coefficients $u_{\boldsymbol{\nu}}^{(k)}, \boldsymbol{\nu} \neq \mathbf{0}$ :

$$
\begin{aligned}
(i \boldsymbol{\omega} \cdot \boldsymbol{\nu}) u_{\boldsymbol{\nu}}^{(0)} & =R_{\boldsymbol{\nu}} \\
(i \boldsymbol{\omega} \cdot \boldsymbol{\nu}) u_{\boldsymbol{\nu}}^{(k)} & =\sum_{k_{1}+k_{2}=k-1} \sum_{\boldsymbol{\nu}_{0}+\boldsymbol{\nu}_{1}+\boldsymbol{\nu}_{2}=\boldsymbol{\nu}} Q_{\boldsymbol{\nu}_{0}} u_{\boldsymbol{\nu}_{1}}^{\left(k_{1}\right)} u_{\boldsymbol{\nu}_{2}}^{\left(k_{2}\right)}, \quad \forall k \geq 1,
\end{aligned}
$$

for all $\boldsymbol{\nu} \neq \mathbf{0}$. Since the 1.h.s. of (2.10) has zero average, one must also impose

$$
\begin{aligned}
0 & =R_{\mathbf{0}} \\
0 & =\sum_{k_{1}+k_{2}=k-1} \sum_{\boldsymbol{\nu}_{0}+\boldsymbol{\nu}_{1}+\boldsymbol{\nu}_{2}=\mathbf{0}} Q_{\boldsymbol{\nu}_{0}} u_{\boldsymbol{\nu}_{1}}^{\left(k_{1}\right)} u_{\boldsymbol{\nu}_{2}}^{\left(k_{2}\right)}=:\left\langle\left[Q u^{2}\right]^{(k-1)}\right\rangle, \quad \forall k \geq 1 .
\end{aligned}
$$

We note that $R_{\mathbf{0}}=P_{\underline{0}}^{(1)} \mathcal{F}_{0}^{(2)} \delta_{0,2}=0$ so there is no problem with the requirement $0=R_{\mathbf{0}}$.

The graphical representation of the coefficients $u_{\nu}^{(k)}$ is almost exactly like in Ref. [13]. We advise the reader to see Section 4 of Ref. 13] for details. The only two essential differences are the following: (1) Here we represent as black bullets the factors $R_{\boldsymbol{\nu}}$, while in Ref. 13] they were associated to $\left(Q^{-1}\right)_{\boldsymbol{\nu}}$. (2) The order of a tree here is given only by the sum of vertices plus the sum of the order labels of the white bullets, while in Ref. [13] the number of black bullets was also counted in the order. There is also here a slight modification of notation: while in Ref. [13] $u_{\mathbf{0}}^{(k)}:=c^{(k)}$, here $u_{\mathbf{0}}^{(k)}:=\alpha^{(k)}$, for all $k \geq 0$. We give below the pertinent definitions of Ref. [13] adapted to our present case.

Definition 2.6 A tree $\theta$ is a connected set of points and lines with no cycle such that all the lines are oriented toward a unique point called the root. We call nodes all the points in a tree except the root. The root only admits one entering line: such a line is called the root line. The orientation of the lines in a tree induces a partial ordering relation between the nodes. We denote by $\preceq$ this relation: given two nodes $v$ and $w$, we shall write $w \preceq v$ every time $v$ is a long the path (of lines) which connects $w$ to the root. Given a tree $\theta$, we can identify in $\theta$ the following subsets.

- $E(\theta)$ : the set of endpoints (final nodes) in $\theta$. A node $v \in \theta$ will be an endpoint if no line enters $v$. We denote by $|E(\theta)|$ the number of endpoints in $\theta$.

- $E_{W}(\theta) \subseteq E(\theta)$ : the set of white bullets in $\theta$. With each $v \in E_{W}(\theta)$ we associate a mode label $\boldsymbol{\nu}_{v}=\mathbf{0}$, an order label $k_{v} \in \mathbb{Z}_{+}$and a node factor $F_{v}=\alpha^{\left(k_{v}\right)}$. We denote by $\left|E_{W}(\theta)\right|$ the number of white bullets in $\theta$.

- $E_{B}(\theta)=E(\theta) \backslash E_{W}(\theta)$ : the set of black bullets in $\theta$. With each $v \in E_{B}(\theta)$ we associate a mode label $\boldsymbol{\nu}_{v} \neq \mathbf{0}$ and $a$ node factor $F_{v}=R_{\boldsymbol{\nu}_{v}}$. We denote by $\left|E_{B}(\theta)\right|$ the number of black bullets in $\theta$.

- $V(\theta)$ : the set of vertices in $\theta$. If $v \in V(\theta)$, then $v$ has at least one entering line. We associate with each vertex $v \in V(\theta)$ a mode label $\boldsymbol{\nu}_{v} \in \mathbb{Z}^{d}$ and a node factor $F_{v}=Q_{\boldsymbol{\nu}_{v}}$. We denote by $|V(\theta)|$ the number of vertices in $\theta$.

- $B(\theta)=E_{B}(\theta) \cup V(\theta)$ : the set of black bullets and vertices in $\theta$. We denote by $|B(\theta)|$ the number of black bullets plus vertices in $\theta$, i.e. $|B(\theta)|=\left|E_{B}(\theta)\right|+|V(\theta)|$.

- $L(\theta)$ : the set of lines in $\theta$. Each line $\ell \in L(\theta)$ leaves a point $v$ and enters another one which we shall denote by $v^{\prime}$. Since $\ell$ is uniquely identified with $v$ (the point which $\ell$ leaves), we may write $\ell=\ell_{v}$. For each line $\ell$ we associate a momentum label $\boldsymbol{\nu}_{\ell} \in \mathbb{Z}^{d}$ and a propagator $g_{\ell}=1 /\left(i \boldsymbol{\omega} \cdot \boldsymbol{\nu}_{\ell}\right)$ if $\boldsymbol{\nu}_{\ell} \neq \mathbf{0}$ and $g_{\ell}=1$ if $\boldsymbol{\nu}_{\ell}=\mathbf{0}$; we say that the momentum $\boldsymbol{\nu}_{\ell}$ flows through the line $\ell$. The 
modes and the momenta are related by the following: if $\ell=\ell_{v}$ and $\ell^{\prime}, \ell^{\prime \prime}$ are the lines entering $v$, then

$$
\boldsymbol{\nu}_{\ell}=\boldsymbol{\nu}_{v}+\boldsymbol{\nu}_{\ell^{\prime}}+\boldsymbol{\nu}_{\ell^{\prime \prime}}=\sum_{\substack{w \in B(\theta) \\ w \preceq v}} \boldsymbol{\nu}_{w} .
$$

We denote by $|L(\theta)|$ the number of lines in $\theta$.

We call equivalent two trees which can be transformed into each other by continuously deforming the lines in such a way that they do not cross each other.

Definition 2.7 Let $\mathcal{T}_{k, \nu}$ be the set of inequivalent trees $\theta$ satisfying:

1. for each vertex $v \in V(\theta)$, there exist exactly two entering lines in $v$;

2. for each line $\ell$ which is not the root line one has $\boldsymbol{\nu}_{\ell}=\mathbf{0}$ if and only if $\ell$ leaves a white bullet;

3. the number of vertices and the sum of all the order labels of the white bullets are such that defining $k_{1}:=|V(\theta)|$ and $k_{2}:=\sum_{v \in E_{W}(\theta)} k_{v}$, we have $k_{1}+k_{2}=k$;

4. the momentum flowing through the root line is $\boldsymbol{\nu}$.

We refer to $\mathcal{T}_{k, \nu}$ as the set of trees of order $k$ and total momentum $\boldsymbol{\nu}$.

Based on the above definitions, we write for all $k \geq 0$ and for all $\boldsymbol{\nu} \in \mathbb{Z}^{d}, \boldsymbol{\nu} \neq \mathbf{0}$ :

$$
u_{\nu}^{(k)}=\sum_{\theta \in \mathcal{T}_{k, \nu}} \operatorname{Val}(\theta),
$$

where Val $: \mathcal{T}_{k, \boldsymbol{\nu}} \rightarrow \mathbb{C}$ is called the value of the tree $\theta$ and it is defined by

$$
\operatorname{Val}(\theta):=\left(\prod_{\ell \in L(\theta)} g_{\ell}\right)\left(\prod_{v \in E(\theta) \cup V(\theta)} F_{v}\right)
$$

where

$$
g_{\ell}:=\left\{\begin{array}{ll}
\frac{1}{i \boldsymbol{\omega} \cdot \boldsymbol{\nu}_{\ell}}, & \boldsymbol{\nu}_{\ell} \neq \mathbf{0}, \\
1, & \boldsymbol{\nu}_{\ell}=\mathbf{0},
\end{array} \quad F_{v}:=\left\{\begin{array}{cc}
Q_{\boldsymbol{\nu}_{v}}, & v \in V(\theta), \\
R_{\boldsymbol{\nu}_{v}}, & v \in E_{B}(\theta), \\
\alpha^{\left(k_{v}\right)}, & v \in E_{W}(\theta) .
\end{array}\right.\right.
$$

All the trees which appear in the expansion of the coefficient $u_{\boldsymbol{\nu}}^{(k)}$ belong to $\mathcal{T}_{k, \boldsymbol{\nu}}$. Reciprocally, every tree in $\mathcal{T}_{k, \boldsymbol{\nu}}$ appears in the graphical expansion of $u_{\boldsymbol{\nu}}^{(k)}$.

It is clear that the constants $u_{\mathbf{0}}^{(k)}=\alpha^{(k)}$ should be recursively fixed from conditions (2.17). We leave this for next section.

\section{Analysis of the zero modes. Fixing $\alpha^{(k)}, k \geq 0$}

We now analyze equations (2.16) and (2.17) in order to fix $\alpha^{(k)}, k \geq 0$. One should keep in mind that these equations are of a recursive nature. Therefore, one first starts by fixing $u_{\boldsymbol{\nu}}^{(0)}, \boldsymbol{\nu} \neq \mathbf{0}$, from (2.16), then one fixes $\alpha^{(0)}$ from (2.17), then one goes back to (2.16) to fix $u_{\boldsymbol{\nu}}^{(1)}, \boldsymbol{\nu} \neq \mathbf{0}$, and so on. Our intention here is to obtain a general recursive expression for the zero modes coefficients $\alpha^{(k)}$. We shall prove that, apart from a spurious situation, the only possible choice of constants $\alpha^{(k)}$ compatible with (2.17) is $\alpha^{(k)}=0$, for all $k \geq 0$. 
Remark 3.1 Let $\theta \in \mathcal{T}_{k, \boldsymbol{\nu}}, k \geq 0, \nu \in \mathbb{Z}^{d}$. Since $k=|V(\theta)|+\sum_{v \in E_{W}(\theta)} k_{v}$, one clearly has $0 \leq|V(\theta)| \leq k$. If, e.g., $E_{W}(\theta)$ contains only one white bullet with order label $k$, then $|V(\theta)|=0$; on the other hand if $E_{W}(\theta)$ contains only white bullets with order label all equal to zero or if it is an empty set, then $|V(\theta)|=k$. Another simple observation is that, by topological reasons, the total number of endpoints of $\theta$ is exactly $|V(\theta)|+1$ (this can be easily proved by induction). So, $\left|E_{W}(\theta)\right|+\left|E_{B}(\theta)\right|=|V(\theta)|+1$ and one has $0 \leq\left|E_{B}(\theta)\right| \leq|V(\theta)|+1$.

Lemma 3.2 In $u_{\nu}^{(k)}, k \geq 0, \boldsymbol{\nu}=\left(\underline{m}, n_{1}, n_{2}\right) \in \mathbb{Z}^{d}, n_{2}$ belongs to the following set of even integers: $\{-2 k,-2(k-1), \ldots,-2,0,2\}$.

Proof. For $k=0, n_{2}=2$ since $u_{\nu}^{(0)} \propto \delta_{n_{2}, 2}$. Now let $k \geq 1$ and $\theta \in \mathcal{T}_{k, \boldsymbol{\nu}}$ be a tree contributing to $u_{\nu}^{(k)}$. With each vertex $v \in V(\theta)$ one associates the factor $\delta_{n_{2}^{(v)},-2}$ in $\operatorname{Val}(\theta)$ and with each black bullet $b \in E_{B}(\theta)$ the factor $\delta_{n_{2}^{(b)}, 2}$. Thus, due to the conservation of momentum (2.18), one must have the constraint $n_{2}=\sum_{v \in V(\theta)} n_{2}^{(v)}+\sum_{b \in E_{B}(\theta)} n_{2}^{(b)}=2\left|E_{B}(\theta)\right|-2|V(\theta)|$ in the root line. From Remark. 3.1] one concludes that $n_{2}=-2 k,-2(k-1), \ldots,-2,0,2$.

Definition 3.3 Let $\theta \in \mathcal{T}_{k, \boldsymbol{\nu}}, k \geq 0, \boldsymbol{\nu} \in \mathbb{Z}^{d}$, such that $E_{W}(\theta)$ is non-empty. Let $\mathcal{A}_{\theta} \subseteq E_{W}(\theta)$ be non-empty. We define $\theta \backslash \mathcal{A}_{\theta}$ as the $\mathcal{A}_{\theta}$-amputated tree generated by amputating the subset $\mathcal{A}_{\theta}$ of white bullets from $\theta$. This means that

$$
\operatorname{Val}\left(\theta \backslash \mathcal{A}_{\theta}\right)=\frac{\operatorname{Val}(\theta)}{\prod_{v \in \mathcal{A}_{\theta}} \alpha^{\left(k_{v}\right)}} \quad \text { and } \quad k_{\theta \backslash \mathcal{A}_{\theta}}=k-\sum_{v \in \mathcal{A}_{\theta}} k_{v},
$$

where $k_{\theta \backslash \mathcal{A}_{\theta}}$ denotes the order of the $\mathcal{A}_{\theta}$-amputated tree. We call amputated line any line coming out from a white bullet in $\mathcal{A}_{\theta}$, after amputation of $\mathcal{A}_{\theta}$. Now let

$$
\mathcal{T}_{k, \boldsymbol{\nu}}^{(p)}:=\left\{\theta \in \mathcal{T}_{k, \boldsymbol{\nu}}: E_{W}(\theta)=\{v\} \text { with } k_{v}=p\right\}
$$

This means that a tree in $\mathcal{T}_{k, \boldsymbol{\nu}}^{(p)}$ has only one white bullet with order label $p$ (and hence $k-p$ vertices). We now amputate the white bullet in $\mathcal{T}_{k, \boldsymbol{\nu}}^{(p)}$; this gives the definition of the set

$$
\widetilde{\mathcal{T}}_{k, \boldsymbol{\nu}}^{(p)}:=\left\{\theta \backslash E_{W}(\theta): \theta \in \mathcal{T}_{k, \boldsymbol{\nu}}^{(p)}\right\}
$$

Of course the order of a tree in $\widetilde{\mathcal{T}}_{k, \boldsymbol{\nu}}^{(p)}$ is equal to its number of vertices, which is just $k-p$. We also introduce here the shorthand: $\widetilde{\mathcal{T}}_{k, \nu}^{(0)}=: \widetilde{\mathcal{T}}_{k, \nu}$, for all $k \geq 1$.

Remark 3.4 From the previous definition and from the fact that $g_{\ell}=1$ when $\ell$ leaves a white bullet, one notes that $\widetilde{\mathcal{T}}_{k, \boldsymbol{\nu}}^{(p)}=\widetilde{\mathcal{T}}_{k-p, \boldsymbol{\nu}}$. Indeed, let $\tilde{\theta} \in \widetilde{\mathcal{T}}_{k, \boldsymbol{\nu}}^{(p)}$ be arbitrary. It follows that there exists $\theta \in \mathcal{T}_{k, \boldsymbol{\nu}}$ with $E_{W}(\theta)=\{v\}$ with $k_{v}=p$ such that $\tilde{\theta}=\theta \backslash E_{W}(\theta)$. This means that $|V(\theta)|=k-p$. Now take $\theta^{\prime} \in \mathcal{T}_{k-p, \nu}$ with $E_{W}\left(\theta^{\prime}\right)=\{v\}$ with $k_{v}=0$ such that $\tilde{\theta}=\theta^{\prime} \backslash E_{W}\left(\theta^{\prime}\right)$. Since $\theta^{\prime} \in \mathcal{T}_{k-p, \nu}^{(0)}$, one finds that $\tilde{\theta} \in \widetilde{\mathcal{T}}_{k-p, \boldsymbol{\nu}}$. Hence $\widetilde{\mathcal{T}}_{k, \boldsymbol{\nu}}^{(p)} \subseteq \widetilde{\mathcal{T}}_{k-p, \boldsymbol{\nu}}$. On the other hand, take an arbitrary $\tilde{\theta} \in \widetilde{\mathcal{T}}_{k-p, \boldsymbol{\nu}}$. Then $\tilde{\theta}=\theta \backslash E_{W}(\theta)$ where $\theta \in \mathcal{T}_{k-p, \nu}$ and $E_{W}(\theta)=\{v\}$ with $k_{v}=0$. Hence, $|V(\theta)|=k-p$, which means that one can take $\theta^{\prime} \in \mathcal{T}_{k, \boldsymbol{\nu}}$ s.t. $E_{W}\left(\theta^{\prime}\right)=\{v\}$ with $k_{v}=p$ and $\tilde{\theta}=\theta^{\prime} \backslash E_{W}\left(\theta^{\prime}\right)$. Since $\theta^{\prime} \in \mathcal{T}_{k, \boldsymbol{\nu}}^{(p)}$, one concludes that $\tilde{\theta} \in \widetilde{\mathcal{T}}_{k, \boldsymbol{\nu}}^{(p)}$. Therefore, $\widetilde{\mathcal{T}}_{k-p, \boldsymbol{\nu}} \subseteq \widetilde{\mathcal{T}}_{k, \boldsymbol{\nu}}^{(p)}$.

Lemma 3.5 Let $k \geq 1$, then $\left\langle\left[Q u^{2}\right]^{(k-1)}\right\rangle=\sum_{p=0}^{k-1} \alpha^{(p)} G_{k-p}$, where, for all $j \geq 1$, $G_{j}:=$ $\sum_{\theta \in \tilde{\mathcal{T}}_{j, 0}} \operatorname{Val}(\theta)$. 
Proof. From the definition,

$$
\left\langle\left[Q u^{2}\right]^{(k-1)}\right\rangle=\sum_{k_{1}+k_{2}=k-1} \sum_{\boldsymbol{\nu}_{0}+\boldsymbol{\nu}_{1}+\boldsymbol{\nu}_{2}=\mathbf{0}} Q_{\boldsymbol{\nu}_{0}} u_{\boldsymbol{\nu}_{1}}^{\left(k_{1}\right)} u_{\boldsymbol{\nu}_{2}}^{\left(k_{2}\right)},
$$

so that by using the definition of tree value (2.20) and the notations of Definition 2.6 one immediately realizes that one can write

$$
\left\langle\left[Q u^{2}\right]^{(k-1)}\right\rangle=\sum_{\theta \in \mathcal{T}_{k, 0}} \operatorname{Val}(\theta) .
$$

Now let $\boldsymbol{\nu}_{1}=\left(\underline{m}_{1}, n_{1}^{(1)}, n_{2}^{(1)}\right) \in \mathbb{Z}^{d}$ and $\boldsymbol{\nu}_{2}=\left(\underline{m}_{2}, n_{1}^{(2)}, n_{2}^{(2)}\right) \in \mathbb{Z}^{d}$. From Lemma 3.2

$$
\begin{aligned}
& n_{2}^{(1)} \in\left\{-2 k_{1},-2\left(k_{1}-1\right), \ldots,-2,0,2\right\} \\
& n_{2}^{(2)} \in\left\{-2 k_{2},-2\left(k_{2}-1\right), \ldots,-2,0,2\right\} .
\end{aligned}
$$

To be more precise, let $\theta_{j} \in \mathcal{T}_{k_{j}, \boldsymbol{\nu}_{j}}, j=1,2$, be a tree contributing to $u_{\boldsymbol{\nu}_{j}}^{\left(k_{j}\right)}$, then $n_{2}^{(1)}=2\left(b_{1}-v_{1}\right)$ and $n_{2}^{(2)}=2\left(b_{2}-v_{2}\right)$, where $b_{j}$ and $v_{j}$ are the number of black bullets and the number of vertices in $\theta_{j}$, respectively. From $\theta_{1}$ and $\theta_{2}$ we would like to construct a tree $\theta \in \mathcal{T}_{k, \mathbf{0}}, k=k_{1}+k_{2}+1$, contributing to $\left\langle\left[Q u^{2}\right]^{(k-1)}\right\rangle$. First one must note that the root lines of $\theta_{1}$ and $\theta_{2}$ enter a vertex in $\theta$ with mode $\boldsymbol{\nu}_{0}=\left(\underline{0}, n_{1}^{(0)},-2\right)$. As the line which exits this vertex (root line) carries zero momentum, one has the constraint $-2+n_{2}^{(1)}+n_{2}^{(2)}=0$. Thus, $\left(b_{1}+b_{2}\right)-\left(v_{1}+v_{2}+1\right)=0$. This last relation implies that $\left|E_{B}(\theta)\right|=|V(\theta)|$, so (see Remark 3.1) the tree $\theta$ contributing to $\left\langle\left[Q u^{2}\right]^{(k-1)}\right\rangle$ must have exactly one white bullet (with some order label $p$ ). Of course $|V(\theta)|+p=k$ and $1 \leq|V(\theta)| \leq k$, hence $0 \leq p \leq k-1$. Therefore, one can write

$$
\begin{aligned}
\left\langle\left[Q u^{2}\right]^{(k-1)}\right\rangle & =\sum_{p=0}^{k-1} \sum_{\theta \in \mathcal{T}_{k, \mathbf{0}}^{(p)}} \operatorname{Val}(\theta)=\sum_{p=0}^{k-1} \sum_{\theta \in \mathcal{T}_{k, \mathbf{0}}^{(p)}} \alpha^{(p)} \operatorname{Val}\left(\theta \backslash E_{W}(\theta)\right) \\
& =\sum_{p=0}^{k-1} \alpha^{(p)} \sum_{\theta \in \tilde{\mathcal{T}}_{k, \mathbf{0}}^{(p)}} \operatorname{Val}(\theta)=\sum_{p=0}^{k-1} \alpha^{(p)} \sum_{\theta \in \tilde{\mathcal{T}}_{k-p, \mathbf{0}}} \operatorname{Val}(\theta) \\
& =: \sum_{p=0}^{k-1} \alpha^{(p)} G_{k-p},
\end{aligned}
$$

where Remark 3.4 was used. Note that, by construction, $G_{j}, j \geq 1$, is expressed by a sum of trees with no white bullets such that they have exactly $j$ black bullets and $j$ vertices.

Definition 3.6 Let $k \geq 1$ and $\widetilde{\mathcal{T}}_{k, \nu}$ as in Definition 3.3. We split $\widetilde{\mathcal{T}}_{k, \nu}$ into two disjoint sets as follows: $\widetilde{\mathcal{T}}_{k, \boldsymbol{\nu}}=: \widetilde{\mathcal{T}}_{k, \boldsymbol{\nu}}^{c} \cup \widetilde{\mathcal{T}}_{k, \boldsymbol{\nu}}^{n c}$, where

- $\widetilde{\mathcal{T}}_{k, \boldsymbol{\nu}}^{c}$ : set of trees in $\widetilde{\mathcal{T}}_{k, \boldsymbol{\nu}}$ such that the amputated line is connected to the root line;

- $\widetilde{\mathcal{T}}_{k, \boldsymbol{\nu}}^{n c}$ : set of trees in $\widetilde{\mathcal{T}}_{k, \boldsymbol{\nu}}$ such that the amputated line is not connected to the root line.

We call a tree in $\widetilde{\mathcal{T}}_{k, \boldsymbol{\nu}}^{c}$ as a c-class tree and a tree in $\widetilde{\mathcal{T}}_{k, \boldsymbol{\nu}}^{\text {nc }}$ as a nc-class tree. Note any nc-class tree has order $k \geq 2$.

Any tree in $\widetilde{\mathcal{T}}_{k, \boldsymbol{\nu}}^{n c}$ can be transformed to be drawn in its "canonical form" as depicted in Figure [1 Indeed, let $\theta \in \widetilde{\mathcal{T}}_{k, \nu}^{n c}, k \geq 2, \boldsymbol{\nu} \in \mathbb{Z}^{d}$, be arbitrary. Let $v_{1}^{\prime}$ be the vertex connected to the amputated line of $\theta$. Define $v_{2}^{\prime}$ as the vertex such that one of its entering lines is exactly the line exiting $v_{1}^{\prime}$. Define 
$v_{j}^{\prime}$ inductively as the vertex such that one of its entering lines is exactly the line exiting $v_{j-1}^{\prime}$. If, for some $j \geq 2, v_{j}^{\prime}$ is the vertex connected to the root line, then we set $n:=j$. Now relabel the $n$ vertices defined above as follows: $v_{j}=v_{n-j+1}^{\prime}, 1 \leq j \leq n$. The vertices $v_{j}$ will be called canonical vertices. Set $\theta_{j}, 1 \leq j \leq n-1$, as the subtree whose root line is the one entering $v_{j}$ and not exiting $v_{j+1} ; \theta_{n}$ is defined as the subtree whose root line enters $v_{n}$, not being the amputated line. The subtrees $\theta_{j}$ will be called canonical subtrees. Now draw the tree in such a way that the root line of each $\theta_{j}, 1 \leq j \leq n$, is the upper line entering the vertex $v_{j}$ : in this way $\theta \in \widetilde{\mathcal{T}}_{k, \boldsymbol{\nu}}^{n c}$ is as represented in Figure $\square$ From now on, any tree in $\widetilde{\mathcal{T}}_{k, \boldsymbol{\nu}}^{n c}$ is thought of as being drawn in its "canonical form".

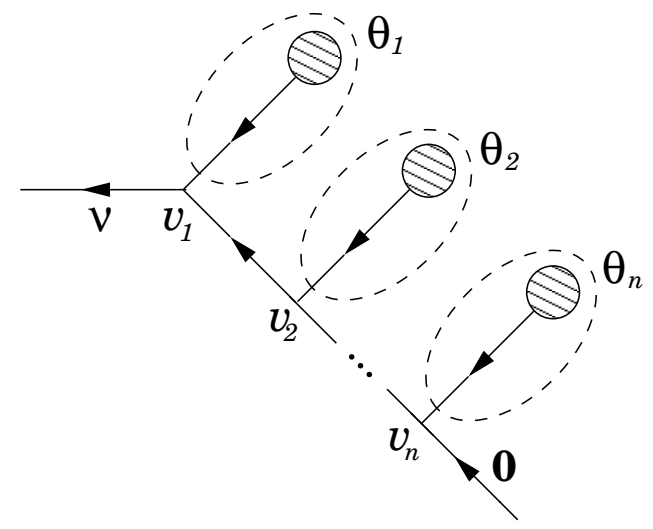

Figure 1: Canonical form of a tree in $\widetilde{\mathcal{T}}_{k, \nu}^{n c}$. The dashed bullet represents a general subtree containing only black bullets. Each canonical subtree $\theta_{j}$, $1 \leq j \leq n$, is of order $k_{\theta_{j}}$ and contains exactly $k_{\theta_{j}}$ vertices and $k_{\theta_{j}}+1$ black bullets. Taking into account the $n$ canonical vertices $\left\{v_{1}, \ldots, v_{2}\right\}$, one has $k=n+\sum_{j=1}^{n} k_{\theta_{j}}$. Note that $2 \leq n \leq k$. The amputation of the white bullet leaves a line with vanishing momentum connected to the vertex $v_{n}$; we call amputated line such a line.

Remark 3.7 Each canonical subtree $\theta_{j} \subset \theta$ defined above gives a contribution to $u_{\boldsymbol{\nu}_{j}}^{\left(k_{\theta_{j}}\right)}$ if $k_{\theta_{j}}$ is the order of $\theta_{j}$ and if $\boldsymbol{\nu}_{j}$ is the momentum flowing through its root line. Of course $\boldsymbol{\nu}_{j} \neq \mathbf{0}$ since this would give a contribution to $\alpha^{\left(k_{j}\right)}$ and white bullets are discarded along the construction. More generally, as each line in $L(\theta)$, which is neither the root line nor the line exiting from the amputated white bullet, can be seen as the root line of a subtree, then it must have a momentum different from zero, as there are no other white bullets.

Remark 3.8 Note that there are $2^{n}$ inequivalent trees in $\widetilde{\mathcal{T}}_{k, \boldsymbol{\nu}}^{n c}$ admitting the same canonical form with $n$ canonical subtrees.

One now writes the value of a canonical subtree $\theta_{j}$ as

$$
\operatorname{Val}\left(\theta_{j}\right)=\frac{b_{\boldsymbol{\nu}_{j}}^{(j)}}{i \boldsymbol{\omega} \cdot \boldsymbol{\nu}_{j}} \equiv B_{\boldsymbol{\nu}_{j}}^{(j)}, \quad \boldsymbol{\nu}_{j} \neq \mathbf{0}, \quad 1 \leq j \leq n
$$

Therefore, $\theta_{j}$ gives a contribution to the function

$$
B_{j}(t)=\int_{0}^{t} \mathrm{~d} t^{\prime} b_{j}\left(t^{\prime}\right)+C_{j}
$$

where the integration constant $C_{j}$ is chosen in such a way that summed to the constant term arising from the definite integral gives the zero Fourier mode of $B_{j}$ :

$$
C_{j}-\sum_{\boldsymbol{\nu} \in \mathbb{Z}_{*}^{d}} \frac{b_{\boldsymbol{\nu}}^{(j)}}{i \boldsymbol{\omega} \cdot \boldsymbol{\nu}}=B_{\mathbf{0}}^{(j)} .
$$

Since $B_{0}^{(j)}$ must be vanishing,

$$
B_{j}(t)=\sum_{\boldsymbol{\nu} \in \mathbb{Z}_{*}^{d}} B_{\boldsymbol{\nu}}^{(j)} e^{i \boldsymbol{\omega} \cdot \boldsymbol{\nu} t}=: \int b_{j},
$$


where the above integral has to be interpreted as a shorthand notation for (3.1) with $C_{j}$ fixed from (3.2) by imposing $B_{\mathbf{0}}^{(j)}=0$. One should think of it as just a zero average primitive of $b_{j}$.

Lemma 3.9 Let $\theta \in \widetilde{\mathcal{T}}_{k, \mathbf{0}}^{n c}$ be a nc-class tree as the one in Figure 1 with order $k \geq 2$ and $2 \leq n \leq k$ canonical subtrees $\theta_{1}, \ldots, \theta_{n}$. Let $\mathcal{N}_{\mathbf{0}}:=\left\{\boldsymbol{\nu}_{v} \in \mathbb{Z}_{*}^{d}: v \in B(\theta)\right\}$ and $a_{j}(t):=Q(t) B_{j}(t), 1 \leq j \leq n$. Then

$$
\sum_{\mathcal{N}_{\mathbf{0}}} \operatorname{Val}(\theta)=\left\langle a_{1} \int a_{2} \int \cdots \int a_{n-1} \int a_{n}\right\rangle,
$$

where all the integrals are in the sense of (3.3).

Proof. Let $1 \leq j \leq n$ and denote by $\boldsymbol{\nu}_{0, j}$ the Fourier mode of the canonical vertex $v_{j}$ and by $\boldsymbol{\nu}_{j}$ the momentum flowing through the root line of the canonical subtree $\theta_{j}$. Note that $\boldsymbol{\nu}_{0, j} \neq \mathbf{0}$, since this would give $Q_{\mathbf{0}}=0$. Also $\boldsymbol{\nu}_{j} \neq \mathbf{0}$ and more generally, $\boldsymbol{\nu}_{\ell} \neq \mathbf{0}$, for all $\ell \in L(\theta)$ different from the root line and the line leaving the amputated white bullet (see Remark 3.7). Now the momentum flowing through the root line is zero, which means that

$$
\sum_{j=1}^{n} \boldsymbol{\nu}_{j}+\sum_{j=1}^{n} \boldsymbol{\nu}_{0, j}=\mathbf{0}
$$

Therefore, by an explicit computation,

$$
\begin{aligned}
\sum_{\mathcal{N}_{\mathbf{0}}} \operatorname{Val}(\theta) & =\prod_{r=1}^{n} \sum_{\boldsymbol{\nu}_{r}} \sum_{\boldsymbol{\nu}_{0, r}} \frac{\prod_{j=1}^{n} Q_{\boldsymbol{\nu}_{0, j}} b_{\boldsymbol{\nu}_{j}}^{(j)}}{\prod_{j=1}^{n}\left(i \boldsymbol{\omega} \cdot \boldsymbol{\nu}_{j}\right) \sum_{p=1}^{j} i \boldsymbol{\omega} \cdot\left(\boldsymbol{\nu}_{n-p+1,0}+\boldsymbol{\nu}_{n-p+1}\right)} \\
& =\prod_{r=1}^{n} \sum_{\boldsymbol{\nu}_{r}} \sum_{\boldsymbol{\nu}_{0, r}} \frac{\prod_{j=1}^{n} Q_{\boldsymbol{\nu}_{0, j}} B_{\boldsymbol{\nu}_{j}}^{(j)}}{\prod_{j=1}^{n} \sum_{p=1}^{j} i \boldsymbol{\omega} \cdot\left(\boldsymbol{\nu}_{n-p+1,0}+\boldsymbol{\nu}_{n-p+1}\right)} \\
& =\left\langle\left(Q B_{1}\right) \int\left(Q B_{2}\right) \int \cdots \int\left(Q B_{n-1}\right) \int\left(Q B_{n}\right)\right\rangle,
\end{aligned}
$$

which proves the statement.

Let $\theta \in \widetilde{\mathcal{T}}_{k, \mathbf{0}}^{n c}$ be a $n c$-class tree as the one in Figure [ with order $k \geq 2$ and $n \leq k$ canonical subtrees $\theta_{1}, \ldots, \theta_{n}$. Of course if some two canonical trees $\theta_{i}, \theta_{j}$ are equivalent, then one gets the same contribution in (3.4) by permuting $a_{i}$ with $a_{j}$. This motivate us to give the following definitions: let $\Theta=\left\{\theta_{1}, \ldots \theta_{n}\right\}$ be the collection of all canonical subtrees of $\theta \in \widetilde{\mathcal{T}}_{k, \mathbf{0}}^{n c}$. We split $\Theta$ into $1 \leq m \leq n$ disjoint subsets $E_{j}, 1 \leq j \leq m$, such that $E_{1}$ is composed by all trees in $\Theta$ which are equivalent to $\theta_{1}$, $E_{2}$ is composed by all trees in $\Theta \backslash E_{1}$ which are equivalent to the first tree of $\Theta \backslash E_{1}$ and so on. In this way, $\Theta=\bigcup_{j=1}^{m} E_{j}$, where each $E_{j}$ collects together all trees which are equivalent to each other. Of course each subset $E_{j}$ contains $r_{j}=\left|E_{j}\right|$ (equivalent) trees such that $\sum_{j=1}^{m} r_{j}=n$. The contribution to (3.4) of all trees within the same $E_{j}$ is denoted by $a_{E_{j}}$, where it represents the function $a_{p}$ associated to the tree $\theta_{p}$ which is equivalent to all trees in $E_{j}$. Now, let $S_{n}$ denote the usual permutation group of $n$ elements. We define $S_{n}^{\Theta}:=S_{n} \backslash\left\{\pi \in S_{n}: \pi(i)=j\right.$ if $i \neq j$ and $\theta_{i}, \theta_{j} \in E_{p}$ for some $\left.1 \leq i, j, p \leq n\right\}$. The set $S_{n}^{\Theta}$ will be called the set of all valid permutations within $\Theta$.

Lemma 3.10 Let $\theta \in \widetilde{\mathcal{T}}_{k, \mathbf{0}}^{n c}$ be a nc-class tree as the one in Figure 1 with order $k \geq 2$ and $n \leq k$ canonical subtrees $\theta_{1}, \ldots, \theta_{n}$. Then

$$
\sum_{\pi \in S_{n}^{\ominus}}\left\langle a_{\pi(1)} \int a_{\pi(2)} \int a_{\pi(3)} \cdots \int a_{\pi(n)}\right\rangle=\frac{(-1)^{n+1}}{r_{1} ! \cdots r_{m} !}\left\langle\frac{\mathrm{d}}{\mathrm{d} t}\left(A_{E_{1}}^{r_{1}} \cdots A_{E_{m}}^{r_{m}}\right)\right\rangle=0,
$$

where $A_{E_{j}}=\int a_{E_{j}}$, for all $1 \leq j \leq m$. 
Proof. First let us assume that all the subtrees $\theta_{1}, \ldots \theta_{n}$ are different. Therefore we have $a_{1} \neq a_{2} \neq$ $\cdots \neq a_{n}$ in (3.4). With this assumption $\Theta=\left\{\theta_{1}, \ldots, \theta_{n}\right\}=\bigcup_{j=1}^{n} E_{j}$ with $E_{j}=\left\{\theta_{j}\right\}$ and $r_{j}=1$ for all $1 \leq j \leq n$. By an integration by parts, one has

$$
\left\langle a_{1} \int a_{2} \int a_{3} \ldots \int a_{n}\right\rangle=-\left\langle\left(\int a_{3} \ldots \int a_{n}\right)\left(a_{2} \int a_{1}\right)\right\rangle,
$$

so that by summing also the term $1 \leftrightarrow 2$ and performing another integration by parts, one obtains

$$
\begin{aligned}
\left\langle a_{1} \int a_{2} \int a_{3} \ldots \int a_{n}\right\rangle & +\left\langle a_{2} \int a_{1} \int a_{3} \ldots \int a_{n}\right\rangle \\
& =-\left\langle\left(\int a_{3} \ldots \int a_{n}\right) \frac{\mathrm{d}}{\mathrm{d} t}\left(A_{1} A_{2}\right)\right\rangle=\left\langle\left(\int a_{4} \ldots \int a_{n}\right)\left(A_{1} A_{2} a_{3}\right)\right\rangle .
\end{aligned}
$$

Note that to construct the derivative of $\left(A_{1} A_{2}\right)$ above we have used the $2 !=2$ permutations of $a_{1}, a_{2}$ : $(123 \cdots n)$ and $(213 \cdots n)$. So, by using also $(132 \cdots n),(312 \cdots n)$ and $(321 \cdots n),(231 \cdots n)$, one gets

$$
\left\langle\left(\int a_{4} \ldots \int a_{n}\right)\left(A_{1} A_{3} a_{2}\right)\right\rangle \text { and }\left\langle\left(\int a_{4} \ldots \int a_{n}\right)\left(A_{3} A_{2} a_{1}\right)\right\rangle .
$$

Therefore, the sum of the $3 !=6$ terms obtained by the permutation of $a_{1}, a_{2}, a_{3}$, gives

$$
\left\langle\left(\int a_{4} \ldots \int a_{n}\right) \frac{\mathrm{d}}{\mathrm{d} t}\left(A_{1} A_{2} A_{3}\right)\right\rangle=-\left\langle\left(\int a_{5} \ldots \int a_{n}\right)\left(A_{1} A_{2} A_{3} a_{4}\right)\right\rangle .
$$

We now go on and sum the $4 !=24$ terms obtained by the permutation of $a_{1}, a_{2}, a_{3}, a_{4}$ to obtain a derivative of $\left(A_{1} A_{2} A_{3} A_{4}\right)$. We iterate this procedure until exhausting the $n$ ! permutations of $a_{1}, \ldots, a_{n}$, giving

$$
\sum_{\pi \in S_{n}}\left\langle a_{\pi(1)} \int a_{\pi(2)} \int a_{\pi(3)} \ldots \int a_{\pi(n)}\right\rangle=(-1)^{n+1}\left\langle\frac{\mathrm{d}}{\mathrm{d} t}\left(A_{1} A_{2} \ldots A_{n}\right)\right\rangle=0,
$$

which is the statement of the lemma in the case where all $a_{j}$ are different.

Now assume the more general situation where $\Theta=\left\{\theta_{1}, \ldots, \theta_{n}\right\}=\bigcup_{j=1}^{m} E_{j}$, for some $m<n$. Then we can permute $i \leftrightarrow j$ iff $a_{i} \neq a_{j}$ (we call this a valid permutation). The set of all valid permutations within $\Theta$ is what we have denoted by $S_{n}^{\Theta}$ above. The total number of valid permutations is $\frac{n !}{r_{1} ! \cdots r_{m} !}$. Therefore, by using the result of last formula, one arrives at the general statement.

Remark 3.11 Note that the cancellation described by Lemma 3.10 occurs at fixed values of the mode labels. In other words, if we consider a fixed set of mode labels in $\mathcal{N}_{0}$ contributing to the sum in (3.5), and hence we replace each $a_{j}=Q B_{j}$ in the last line with the corresponding harmonic $a_{j, \nu_{j}} e^{i \boldsymbol{\omega} \cdot \boldsymbol{\nu}_{j} t}$, we immediately realize that the argument given in the proof applies unchanged.

Lemma 3.12 For all $k \geq 2$ one has the identity $\sum_{\theta \in \tilde{\mathcal{T}}_{k, \mathbf{0}}^{n c}} \operatorname{Val}(\theta)=0$. Therefore, for all $j \geq 1$, $G_{j}=\sum_{\theta \in \tilde{\mathcal{T}}_{j, 0}^{c}} \operatorname{Val}(\theta)$.

Proof. Let $k \geq 2$. The result follows by a combination of Lemma 3.9 and Lemma 3.10 Indeed, the sum of all possible trees in $\widetilde{\mathcal{T}}_{k, \mathbf{0}}^{n c}$ (including the sum over the Fourier modes) means that we have to sum all valid permutations of $a_{1}, \ldots, a_{n}$ in (3.4) for all trees with $2 \leq n \leq k$ canonical subtrees. Since this sum gives the average of a total derivative, one concludes that $\sum_{\theta \in \widetilde{T}_{k, 0}^{n c}} \operatorname{Val}(\theta)=0$. Now, since $\widetilde{\mathcal{T}}_{1,0}$ contain only $c$-class trees, one concludes that

$$
G_{j}=\sum_{\theta \in \tilde{\mathcal{T}}_{j, \mathbf{0}}} \operatorname{Val}(\theta)=\sum_{\theta \in \tilde{\mathcal{T}}_{j, \mathbf{0}}^{c}} \operatorname{Val}(\theta)+\sum_{\theta \in \tilde{\mathcal{T}}_{j, \mathbf{0}}^{n c}} \operatorname{Val}(\theta)=\sum_{\theta \in \tilde{\mathcal{T}}_{j, \mathbf{0}}^{c}} \operatorname{Val}(\theta),
$$

for all $j \geq 1$. 
Proposition 3.13 Let $G_{j}, j \geq 1$, be as the previous lemma. Suppose that $G_{j_{0}} \neq 0$ for some $j_{0} \geq 1$. Then, 2.17) holds iff $\alpha^{(k)}=0$ for all $k \geq 0$.

Proof. By Lemma 3.5 condition (2.17) reads

$$
0=\left\langle\left[Q u^{2}\right]^{(k-1)}\right\rangle=\sum_{p=0}^{k-1} \alpha^{(p)} G_{k-p}, \quad \forall k \geq 1
$$

We shall prove by induction that $\alpha^{(p)}=0, p \geq 0$, is the unique solution of (3.6) if $G_{j_{0}} \neq 0$ for some $j_{0} \geq 1$. Indeed, let $j_{0} \geq 1$ be such that $G_{1}=\cdots=G_{j_{0}-1}=0$ and $G_{j_{0}} \neq 0$. Then, equation (3.6) is automatically satisfied for all $1 \leq k \leq j_{0}-1$. For $k=j_{0}$, one has

$$
0=\alpha^{(0)} G_{j_{0}}+\sum_{p=0}^{j_{0}-1} \alpha^{(p)} G_{j_{0}-p}=\alpha^{(0)} G_{j_{0}}
$$

Therefore, $\alpha^{(0)}=0$. Now suppose that $\alpha^{(0)}=\cdots=\alpha^{\left(k_{0}\right)}=0$ for some $k_{0} \geq 1$ and let us prove that $\alpha^{\left(k_{0}+1\right)}=0$. Using (3.6) for $k=j_{0}+k_{0}+1$, we have

$$
0=\sum_{p=0}^{j_{0}+k_{0}} \alpha^{(p)} G_{j_{0}+k_{0}+1-p}=\sum_{p=0}^{k_{0}} \alpha^{(p)} G_{j_{0}+k_{0}+1-p}+\sum_{p=k_{0}+1}^{j_{0}+k_{0}} \alpha^{(p)} G_{j_{0}+k_{0}+1-p}=\alpha^{\left(k_{0}+1\right)} G_{j_{0}},
$$

which implies that $\alpha^{\left(k_{0}+1\right)}=0$.

Remark 3.14 Note that one can always suppose that the function $p_{1}$ in 1.1) has zero average (i.e. $\left.P_{\underline{0}}^{(1)}=0\right)$, by an appropriate choice of the average of $p_{0}$. In such a case, since one has

$$
G_{1}=\left\langle Q \int R\right\rangle=2 \sum_{n_{1} \in \mathbb{Z}} P_{\underline{0}}^{(1)} \frac{\mathcal{F}_{n_{1}}^{(-2)} \mathcal{F}_{-n_{1}}^{(2)}}{i\left(2 \Omega_{0}-n_{1} \omega_{0}\right)}
$$

one finds $G_{1}=0$. This shows that it is important to consider the possibility that the first non-vanishing $G_{j}$ has $j>1$.

Proposition 3.15 Let $G_{j}, j \geq 1$, be as the previous lemma. Then,

(a) $\langle Q u\rangle=\frac{1}{2} \sum_{k=0}^{\infty} \varepsilon^{k} G_{k+1}$.

(b) $\Omega_{\varepsilon}=\Omega_{0} \Leftrightarrow G_{k}=0, \forall k \geq 1$.

(c) $\Omega_{\varepsilon} \in \mathbb{R} \Leftrightarrow \overline{G_{k}}=-G_{k}, \forall k \geq 1$.

In (a) the equality is in the sense of formal power series (that is it holds order by order).

Proof. Let us first write $\langle Q u\rangle$ in Fourier space:

$$
\langle Q u\rangle=\sum_{k=0}^{\infty} \varepsilon^{k}\left\langle[Q u]^{(k)}\right\rangle, \quad \text { where } \quad\left\langle[Q u]^{(k)}\right\rangle=\sum_{\boldsymbol{\nu}_{0}+\boldsymbol{\nu}_{1}=\mathbf{0}} Q_{\boldsymbol{\nu}_{0}} u_{\boldsymbol{\nu}_{1}}^{(k)}, \quad \forall k \geq 1
$$

Now let $\boldsymbol{\nu}_{1}=\left(\underline{m}_{1}, n_{1}^{(1)}, n_{2}^{(1)}\right) \in \mathbb{Z}^{d}$. Of course $\boldsymbol{\nu}_{1} \neq \mathbf{0}$ since $\boldsymbol{\nu}_{0}=-\boldsymbol{\nu}_{1}$ and $Q_{\mathbf{0}}=0$. From Lemma 3.2

$$
n_{2}^{(1)} \in\{-2 k,-2(k-1), \ldots,-2,0,2\} .
$$


To be more precise, let $\theta_{1} \in \mathcal{T}_{k, \nu_{1}}$ be a tree contributing to $u_{\boldsymbol{\nu}_{1}}^{(k)}$; then $n_{2}^{(1)}=2\left(b_{1}-v_{1}\right)$, where $b_{1}$ and $v_{1}$ are the number of black bullets and the number of vertices in $\theta_{1}$, respectively. From $\theta_{1}$ we would like to construct a tree $\theta \in \mathcal{T}_{k+1,0}$ contributing to $\left\langle[Q u]^{(k)}\right\rangle$. We do this as follows. Take the root line of $\theta_{1}$ entering a vertex $v$ with mode $\boldsymbol{\nu}_{0}=\left(\underline{0}, n_{1}^{(0)},-2\right)$. Add a line, with zero momentum, entering such a vertex. We do not associate any propagator with this line, which means that it works as an amputated line (we call this the amputated line of $\theta$ ); note that we can consider such a tree as a tree amputated of a white bullet. Finally, we let the root line of $\theta$ be the line exiting the vertex $v$ carrying zero momentum. Thus, $\boldsymbol{\nu}_{0}+\boldsymbol{\nu}_{1}=\mathbf{0}$. This last relation implies that $-2+n_{2}^{(1)}=0$, which means that $b_{1}-\left(v_{1}+1\right)=0$. Therefore, $\left|E_{B}(\theta)\right|=|V(\theta)|$, leading to the conclusion that $\theta$ must have only one endpoint which is not a black bullet (see Remark 3.1). This leaves room only for the amputated line of $\theta$, so that $\theta_{1}$ must have only black bullets. Finally, one concludes that if $\theta$ contributes to $\left\langle[Q u]^{(k)}\right\rangle$,

then $\theta \in \widetilde{\mathcal{T}}_{k+1, \mathbf{0}}^{c}$. On the other hand, only half of the trees in $\widetilde{\mathcal{T}}_{k+1, \mathbf{0}}^{c}$ contributes to $\left\langle[Q u]^{(k)}\right\rangle$ since in $\widetilde{\mathcal{T}}_{k+1, \mathbf{0}}^{c}$ we take into account two possibilities for amputating the leg connected to the root line. Therefore, by Lemma 3.12 one can write

$$
\left\langle[Q u]^{(k)}\right\rangle=\frac{1}{2} \sum_{\theta \in \widetilde{\mathcal{T}}_{k+1,0}^{c}} \operatorname{Val}(\theta)=\frac{1}{2} G_{k+1} \quad \text { and } \quad\langle Q u\rangle=\frac{1}{2} \sum_{k=0}^{\infty} \varepsilon^{k} G_{k+1},
$$

where the last formula holds as equality between formal series. This proves (a). Items (b) and (c) follows immediately from (a) remembering that $\Omega_{\varepsilon}=\Omega_{0}+\langle g\rangle=\Omega_{0}+i \varepsilon\langle Q u\rangle$.

\section{Analysis of the non-renormalized expansion}

One of the main results of last section (see Proposition 3.13) tell us that all $\alpha^{(k)}=0$ if some $G_{j_{0}} \neq 0$, a condition which we henceforth assume; we shall come back to this in the last section. Therefore, one should not worry about white bullets and trivial propagators. For all $k \geq 0$ and all $\boldsymbol{\nu} \in \mathbb{Z}_{*}^{d}$, define $\mathscr{T}_{k, \boldsymbol{\nu}}:=\left\{\theta \in \mathcal{T}_{k, \nu}: E_{W}(\theta)=\emptyset\right\}$. Then,

$$
u_{\nu}^{(k)}=\sum_{\theta \in \mathscr{T}_{k, \boldsymbol{\nu}}} \operatorname{Val}(\theta), \quad \operatorname{Val}(\theta)=\left(\prod_{\ell \in L(\theta)} g_{\ell}\right)\left(\prod_{v \in B(\theta)} F_{v}\right),
$$

where

$$
g_{\ell}=\frac{1}{i \boldsymbol{\omega} \cdot \boldsymbol{\nu}_{\ell}}, \quad \boldsymbol{\nu}_{\ell} \in \mathbb{Z}_{*}^{d} \quad \text { and } \quad F_{v}=\left\{\begin{array}{ll}
Q_{\boldsymbol{\nu}_{v}}, & v \in V(\theta) \\
R_{\boldsymbol{\nu}_{v}}, & v \in E_{B}(\theta)
\end{array} .\right.
$$

Moreover, $u_{\mathbf{0}}^{(k)}=\alpha^{(k)}=0$ for all $k \geq 0$.

Lemma 4.1 Let $k \geq 0$ and $\boldsymbol{\nu} \in \mathbb{Z}_{*}^{d}$, then $\left|u_{\nu}^{(k)}\right| \leq A B^{k}(k !)^{\beta} e^{-\kappa^{\prime}|\boldsymbol{\nu}|}$, for positive constants $A, B, \beta$ and $\kappa^{\prime}<\kappa$.

Proof. Let $\theta \in \mathscr{T}_{k, \boldsymbol{\nu}}, k \geq 0, \boldsymbol{\nu} \in \mathbb{Z}_{*}^{d}$. From (4.1), (4.2), (2.15) (see Remark 2.5) and from the Diophantine condition (2.14) (see Remark 2.4), we write

$$
|\operatorname{Val}(\theta)| \leq \mathcal{Q}^{|B(\theta)|} C_{0}^{-|L(\theta)|}\left(\prod_{\ell \in L(\theta)}\left|\boldsymbol{\nu}_{\ell}\right|^{\tau}\right)\left(\prod_{v \in B(\theta)} e^{-\kappa\left|\boldsymbol{\nu}_{v}\right|}\right) .
$$

Now, since $|B(\theta)|=|L(\theta)|$ and

$$
\sum_{\ell \in L(\theta)}\left|\boldsymbol{\nu}_{\ell}\right|=\sum_{\ell \in L(\theta)}\left|\sum_{\substack{w \in B(\theta) \\ w \preceq v: \ell=\ell_{v}}} \boldsymbol{\nu}_{w}\right| \leq \sum_{\ell \in L(\theta)} \sum_{w \in B(\theta)}\left|\boldsymbol{\nu}_{w}\right|=|L(\theta)| \sum_{v \in B(\theta)}\left|\boldsymbol{\nu}_{v}\right|,
$$


it follows that

$$
\prod_{v \in B(\theta)} e^{-\kappa\left|\boldsymbol{\nu}_{v}\right|} \leq\left(\prod_{v \in B(\theta)} e^{-\kappa\left|\boldsymbol{\nu}_{v}\right| / 2}\right)\left(\prod_{\ell \in L(\theta)} e^{-\kappa\left|\boldsymbol{\nu}_{\ell}\right| / 2|L(\theta)|}\right) .
$$

On the other hand, for each line $\ell \in L(\theta)$, one has ${ }^{1}$

$$
\left|\boldsymbol{\nu}_{\ell}\right|^{\tau} e^{-\kappa\left|\nu_{\ell}\right| / 2|L(\theta)|} \leq \tau !\left(\frac{2|L(\theta)|}{\kappa}\right)^{\tau}
$$

Hence

$$
\left(\prod_{\ell \in L(\theta)}\left|\boldsymbol{\nu}_{\ell}\right|^{\tau}\right)\left(\prod_{\ell \in L(\theta)} e^{-\kappa\left|\boldsymbol{\nu}_{\ell}\right| / 2|L(\theta)|}\right) \leq(\tau !)^{|L(\theta)|}\left(\frac{2|L(\theta)|}{\kappa}\right)^{\tau|L(\theta)|},
$$

which, together with the fact that for all $\theta \in \mathscr{T}_{k, \nu}$ one has $|L(\theta)|=|B(\theta)|=2 k+1$, yields

$$
\begin{aligned}
|\operatorname{Val}(\theta)| & \leq\left(\frac{\mathcal{Q} 2^{\tau} \tau !}{C_{0} \kappa^{\tau}}\right)^{2 k+1}\left((2 k+1)^{2 k+1}\right)^{\tau}\left(\prod_{v \in B(\theta)} e^{-\kappa\left|\boldsymbol{\nu}_{v}\right| / 2}\right) \\
& \leq \Gamma_{1} \Gamma_{2}^{k}(k !)^{\beta} e^{-\kappa|\boldsymbol{\nu}| / 4}\left(\prod_{v \in B(\theta)} e^{-\kappa\left|\boldsymbol{\nu}_{v}\right| / 4}\right)
\end{aligned}
$$

where $\Gamma_{1}, \Gamma_{2}, \beta$ are suitable positive constants. In the last step above we used the well known Stirling relation to express $k^{k}$ in terms of $k$ ! and the fact that

$$
\sum_{v \in B(\theta)}\left|\boldsymbol{\nu}_{v}\right| \geq\left|\sum_{v \in B(\theta)} \boldsymbol{\nu}_{v}\right|=|\boldsymbol{\nu}|
$$

We now take into account the fact that the number of trees of fixed order is bounded by $\Gamma_{3}^{k}$, for some positive constant $\Gamma_{3}[13]$. Thus, we finally obtain

$$
\left|u_{\nu}^{(k)}\right| \leq \sum_{\theta \in \mathscr{T}_{k, \boldsymbol{\nu}}}|\operatorname{Val}(\theta)| \leq \Gamma_{1}^{\prime} \Gamma_{2}^{k} \Gamma_{3}^{k}(k !)^{\beta} e^{-\kappa|\boldsymbol{\nu}| / 4}
$$

with $\Gamma_{1}^{\prime}$ a suitable positive constant. This completes the proof of the lemma.

\section{Renormalization}

The main problem with the previous proof is that it does not treat conveniently the small denominators $1 / i \boldsymbol{\omega} \cdot \boldsymbol{\nu}_{\ell}$ which appear in the expansion through the propagators $g_{\ell}$. As a result, we end up with a crude estimate for the coefficients $u_{\boldsymbol{\nu}}^{(k)}$, which complicates the task of studying the absolute convergence of the series for $u$.

To overcome the problem of small denominators, we shall adopt a method well known from the analysis of the Lindstedt series for KAM type problems (see Ref. [15] and references therein). All the complication lies in the fact that $\boldsymbol{\omega} \cdot \boldsymbol{\nu}$ can be arbitrarily small for certain $\boldsymbol{\nu}$ with sufficiently large $|\boldsymbol{\nu}|$. The idea, then, is to separate the "small" parts of $\boldsymbol{\omega} \cdot \boldsymbol{\nu}$ and to resume the corresponding terms in a suitable form, obtaining then a result which can be better estimated. The process of "separation" of the "small" parts of $\boldsymbol{\omega} \cdot \boldsymbol{\nu}$ is implemented via a technique known as the multiscale decomposition of the propagators. We stress that this technique is genuine from methods of the Renormalization Group introduced to deal with related problems in field theories.

\footnotetext{
${ }^{1}$ Due to the inequality $|x|^{\sigma} \leq e^{\delta|x|}(\sigma / e \delta)^{\sigma}, \forall x \in \mathbb{C}$, and to the Stirling relation.
} 


\subsection{Multiscale decomposition of the propagators}

We begin by introducing a bounded non-decreasing $C^{\infty}(\mathbb{R})$ function $\psi(x)$, defined in $\mathbb{R}_{+}$, such that

$$
\psi(x)= \begin{cases}1, & \text { for } x \geq C_{1}, \\ 0, & \text { for } x \leq C_{1} / 2,\end{cases}
$$

where $C_{1} \leq C_{0}$ is to be fixed, with $C_{0}$ the Diophantine constant which appears in (2.14), and setting $\chi(x):=1-\psi(x)$. An example of $\chi(x)$ and $\psi(x)$ with the above properties is found in Figure 2 also define, for all $n \in \mathbb{Z}_{+}, \chi_{n}(x):=\chi\left(2^{n} x\right)$ and $\psi_{n}(x):=\psi\left(2^{n} x\right)$. It is clear that $\chi_{0}(x)=\chi(x)$, $\psi_{0}(x)=\psi(x)$ and $\psi_{n}(x)+\chi_{n}(x)=1, \forall n \geq 0$.

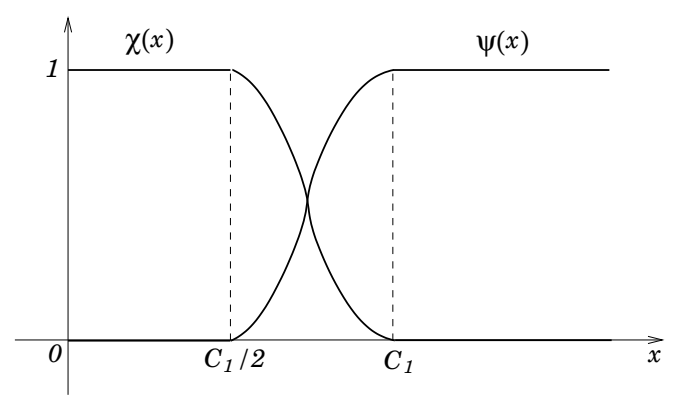

Figure 2: Possible graphs of the functions $\chi(x)$ and $\psi(x)$. Note that $\psi(x)+\chi(x)=1$.

Functions $\chi_{n}(x)$ and $\psi_{n}(x)$ allow us to write the propagator $g_{\ell}$, for all $\ell \in L(\theta)$ and $\boldsymbol{\nu}_{\ell} \neq \mathbf{0}$, as ${ }^{2}$

$$
g_{\ell}=\frac{1}{i \boldsymbol{\omega} \cdot \boldsymbol{\nu}_{\ell}}=\frac{\psi_{0}\left(\left|\boldsymbol{\omega} \cdot \boldsymbol{\nu}_{\ell}\right|\right)}{i \boldsymbol{\omega} \cdot \boldsymbol{\nu}_{\ell}}+\sum_{n=1}^{\infty} \frac{\psi_{n}\left(\left|\boldsymbol{\omega} \cdot \boldsymbol{\nu}_{\ell}\right|\right) \chi_{n-1}\left(\left|\boldsymbol{\omega} \cdot \boldsymbol{\nu}_{\ell}\right|\right)}{i \boldsymbol{\omega} \cdot \boldsymbol{\nu}_{\ell}}
$$

We can still write

$$
\begin{aligned}
& g_{\ell}=\sum_{n=0}^{\infty} g_{\ell}^{(n)}, \\
& g_{\ell}^{(0)}:=\frac{\psi_{0}\left(\left|\boldsymbol{\omega} \cdot \boldsymbol{\nu}_{\ell}\right|\right)}{i \boldsymbol{\omega} \cdot \boldsymbol{\nu}_{\ell}}, \\
& g_{\ell}^{(n)}:=\frac{\psi_{n}\left(\left|\boldsymbol{\omega} \cdot \boldsymbol{\nu}_{\ell}\right|\right) \chi_{n-1}\left(\left|\boldsymbol{\omega} \cdot \boldsymbol{\nu}_{\ell}\right|\right)}{i \boldsymbol{\omega} \cdot \boldsymbol{\nu}_{\ell}}, \quad \forall n \geq 1 .
\end{aligned}
$$

We set $g_{\ell}^{(n)}=g^{(n)}\left(\boldsymbol{\omega} \cdot \boldsymbol{\nu}_{\ell}\right)$

Remark 5.1 Note that for fixed $x=\boldsymbol{\omega} \cdot \boldsymbol{\nu}$, we have $g^{(n)}(x) \neq 0$ only for two values of $n$. This means that the series (5.1) is, in fact, finite. Note also that $g^{(n)}(x) \neq 0$ only if $2^{-n-1} C_{1}<|x|<2^{-n+1} C_{1}$ for $n \geq 1$ and only if $|x|>2^{-1} C_{1}$ for $n=0$. Hence,

$$
g_{\ell}^{(n)} \neq 0 \Rightarrow\left|g_{\ell}^{(n)}\right| \leq C_{1}^{-1} 2^{n+1}
$$

To each line $\ell \in L(\theta)$ with $\boldsymbol{\nu}_{\ell} \neq \mathbf{0}$ we associate a new label $n_{\ell}=0,1,2, \ldots$ called the scale label of line $\ell$. It is important to stress, based on Remark 5.1 that the scale label $n_{\ell}$ of a line $\ell$ tells, essentially, what is the size of the associated propagator $g_{\ell}^{\left(n_{\ell}\right)}$. This is an useful device for "isolating" the contribution of trees containing propagators with too large scales. We shall do this carefully in what follows.

\footnotetext{
${ }^{2}$ Due to the identity $\psi_{0}(x)+\sum_{n=1}^{\infty} \psi_{n}(x) \chi_{n-1}(x)=1$, for all $x \in \mathbb{R}_{+}$(see Remark 5.1 below).
} 
Definition 5.2 We define $\Theta_{k, \nu}$ as the set of trees which differ from those in $\mathscr{T}_{k, \boldsymbol{\nu}}$ by the introduction of the scale labels in the propagators.

With the above definitions, expression (4.1) now reads as

$$
u_{\nu}^{(k)}=\sum_{\theta \in \Theta_{k, \nu}} \operatorname{Val}(\theta), \quad \operatorname{Val}(\theta)=\left(\prod_{\ell \in L(\theta)} g_{\ell}^{\left(n_{\ell}\right)}\right)\left(\prod_{v \in B(\theta)} F_{v}\right),
$$

where the sum over all the trees in $\Theta_{k, \nu}$ implies a further sum over all the possible scale labels for each one of the propagators. Thus, for all $\theta \in \Theta_{k, \nu}$, if $N_{n}(\theta)$ denotes the number of lines in $\theta$ on scale $n$, by using (5.3), (5.2), (2.15) and the fact that $|L(\theta)|=|B(\theta)|=2 k+1$, we obtain

$$
\begin{aligned}
|\operatorname{Val}(\theta)| & \leq\left(2 C_{1}^{-1} \mathcal{Q}\right)^{2 k+1}\left(\prod_{v \in B(\theta)} e^{-\kappa\left|\boldsymbol{\nu}_{v}\right|}\right)\left(\prod_{n=0}^{\infty} 2^{n N_{n}(\theta)}\right) \\
& \leq\left(2 C_{1}^{-1} \mathcal{Q} 2^{n_{1}}\right)^{2 k+1} e^{-\kappa \sum_{v \in B(\theta)}\left|\boldsymbol{\nu}_{v}\right|}\left(\prod_{n=n_{1}}^{\infty} 2^{n N_{n}(\theta)}\right)
\end{aligned}
$$

where in the last step we have introduced a (so far) arbitrary positive integer $n_{1}$ and used the obvious fact that $N_{n}(\theta) \leq|L(\theta)|=2 k+1, \forall n \geq 0$.

Our problem now is to estimate $N_{n}(\theta)$. To solve this, we need to introduce some useful definitions.

Definition 5.3 (Cluster) $A$ cluster $T$ on scale $n$ is a maximal connected subset of a tree $\theta$ such that all its lines have scale $n^{\prime} \leq n$ and there is at least one line on scale $n$. The lines entering a cluster $T$ and the one (if any) exiting it are called the external lines of $T$. Given a cluster $T$ on scale $n$, we denote by $n_{T}=n$ the scale of $T$. Moreover, $V(T), E_{B}(T), B(T)$, and $L(T)$ denote, respectively, the set of vertices, black bullets, vertices plus black bullets, and lines contained in $T$; the external lines of $T$ do not belong to $L(T)$. We finally define the momentum of the cluster $T$ as $\boldsymbol{\nu}_{T}:=\sum_{v \in B(T)} \boldsymbol{\nu}_{v}$. We shall call $k_{T}:=|V(T)|$ the order of T. Some examples of clusters are presented in Figure 3 .

Definition 5.4 (Self-Energy Graph) We call self-energy graph any cluster $T$ of a tree $\theta$ which satisfies

1. $T$ has only one entering line $\ell_{T}^{\text {in }}$ and only one exiting line $\ell_{T}^{\text {out }}$;

2. The momentum of $T$ is zero, i.e. $\boldsymbol{\nu}_{T}=\sum_{v \in B(T)} \boldsymbol{\nu}_{v}=\mathbf{0}$. This means that $\boldsymbol{\nu}_{\ell_{T}^{\text {in }}}=\boldsymbol{\nu}_{\ell_{T}^{\text {out }}}$.

We call self-energy line any line $\ell_{T}^{\text {out }}$ which exits from a self-energy graph $T$. We call normal line any line which is not a self-energy line. Note that if $T$ is a self-energy graph, then $\ell_{T}^{\text {in }} \ell_{T}^{\text {out }} \notin L(T)$, so that $|L(T)|=2 k_{T}-1$ and $|B(T)|=2 k_{T}$. Some examples of self-energy graphs are depicted in Figure 4

Remark 5.5 It is important to stress that due to the condition $\boldsymbol{\nu}_{\ell_{T}^{\text {in }}}=\boldsymbol{\nu}_{\ell_{T}^{\text {out }}}$, the scales on the entering and exiting lines of a self-energy graph $T$ must differ at most by one unit, i.e. $\left|n_{\ell_{T}^{\text {in }}}-n_{\ell_{T}^{\text {out }}}\right| \leq 1$ (see Remark 5.11). Moreover, due to the fact that $T$ defines a cluster, we must have $n_{T}+1 \leq \min \left\{n_{\ell_{T}^{\text {in }}}, n_{\ell_{T}^{\text {out }}}\right\}$, which is equivalent of saying that all the lines within $T$ have scale strictly less then the scale on the external lines $\ell_{T}^{\text {in }}$ and $\ell_{T}^{\text {out }}$.

Due to the presence of self-energy graphs one can have accumulation of small divisors. The heuristic explanation for this is as follows: imagine we have a line $\ell$ on a large scale $n_{\ell} \gg 1$ entering a self-energy graph $T$. This line $\ell^{\prime}$ exiting from $T$ could enter another self-energy graph $T^{\prime}$. Note that such a line $\ell^{\prime}$ 

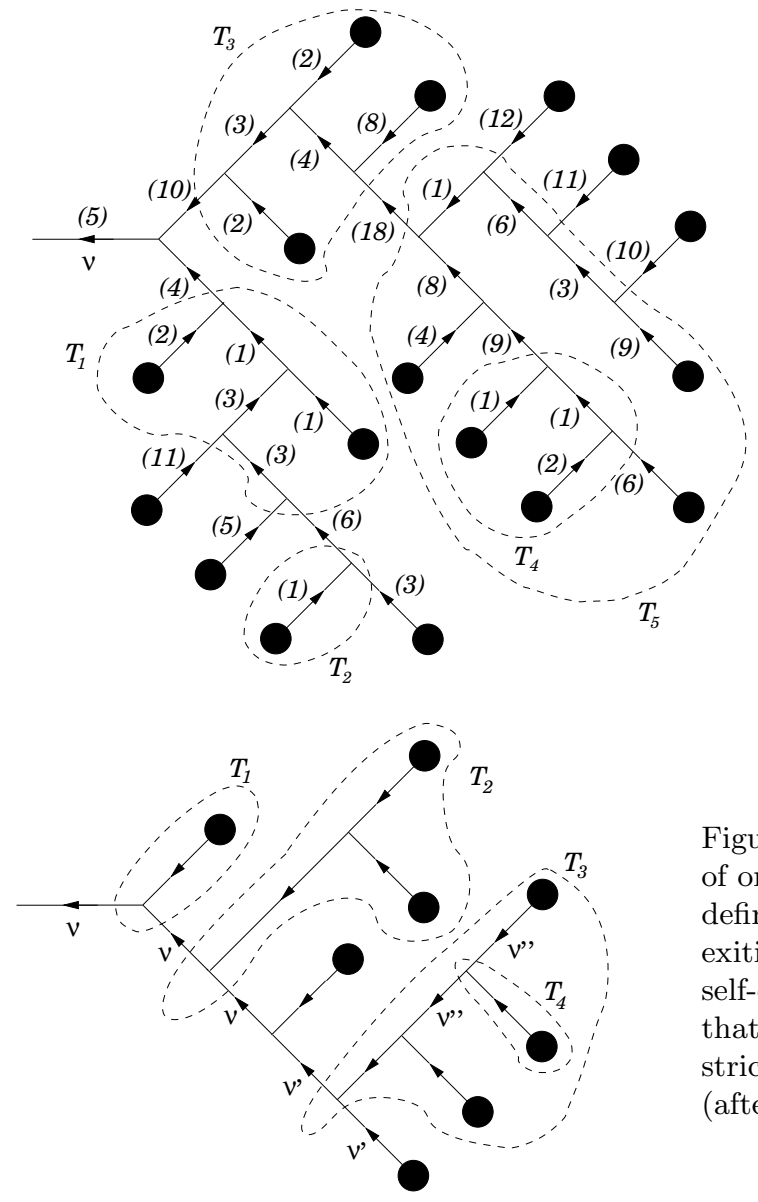

Figure 3: Examples of clusters in a tree of order $k=16$. The number between parentheses above each line denotes the scale of the propagator. Thus, we have $n_{T_{1}}=3, n_{T_{2}}=1$, $n_{T_{3}}=8, n_{T_{4}}=2$ and $n_{T_{5}}=9$. Note that $T_{4} \subset T_{5}$ and therefore $n_{T_{4}}<n_{T_{5}}$. Of course there are other clusters in the example considered which are not shown.

is also on scale $n_{\ell^{\prime}} \gg 1$. This process could repeat itself several times, resulting at the end in a bunch of lines $\ell_{1}, \ldots, \ell_{N}$ on scales $n_{\ell_{i}} \gg 1$, i.e. we end up with an accumulation of small divisors ${ }^{3}$.

Actually, from a more precise point of view, the whole problem with the self-energy graphs is that we are not able to give a satisfactory bound on the number of self-energy lines in a given tree $\theta$. On the other hand, it is easy to show that (see, e.g., Ref. [12]) if we denote by $N_{n}^{\text {norm }}(\theta)$ the number of normal lines in a tree $\theta$, then there exists a positive constant $c$ such that

$$
N_{n}^{\text {norm }}(\theta) \leq c 2^{-n / \tau} \sum_{v \in B(\theta)}\left|\boldsymbol{\nu}_{v}\right|,
$$

where $\tau$ is one of the Diophantine constants appearing in (2.14). Thus, suppose we could neglect all the self-energy lines within any tree $\theta$, i.e. suppose that we could substitute $N_{n}(\theta)$ in (5.4) by $N_{n}^{\text {norm }}(\theta)$ with the above estimate. Then, we would have

$$
|\operatorname{Val}(\theta)| \leq\left(2 C_{1}^{-1} \mathcal{Q} 2^{n_{1}}\right)^{2 k+1} e^{-\kappa \sum_{v \in B(\theta)}\left|\boldsymbol{\nu}_{v}\right|} e^{\left(c \log 2 \sum_{n=n_{1}}^{\infty} n 2^{-n / \tau}\right) \sum_{v \in B(\theta)}\left|\boldsymbol{\nu}_{v}\right|},
$$

for all $n_{1} \geq 0$. Thus, picking $n_{1}=n_{1}(\kappa, c, \tau)$ such that ${ }^{4}$

$$
-\frac{\kappa}{2}+c \log 2 \sum_{n=n_{1}}^{\infty} n 2^{-n / \tau}<0,
$$

\footnotetext{
${ }^{3}$ We remind that the "size" of a propagator grows exponentially with its scale (see Remark [5.1).

${ }^{4}$ Note that this is always possible by choosing $n_{1}$ sufficiently large due to the fact that the sum over $n$ above is convergent and exponentially small in $n_{1}$.
} 
we would obtain

$$
|\operatorname{Val}(\theta)| \leq\left(2 C_{1}^{-1} \mathcal{Q} 2^{n_{1}(\kappa, c, \tau)}\right)^{2 k+1} e^{-\frac{\kappa}{2} \sum_{v \in B(\theta)}\left|\boldsymbol{\nu}_{v}\right|} \leq \Lambda_{1} \Lambda_{2}^{k} e^{-\kappa|\boldsymbol{\nu}| / 4}\left(\prod_{v \in B(\theta)} e^{-\kappa\left|\boldsymbol{\nu}_{v}\right| / 4}\right),
$$

where, in the last inequality, we have used (4.6). Therefore, summing over all the trees (whose number grows at most as $\Lambda_{3}^{k}$, for some positive $\Lambda_{3}$ ) and all the Fourier labels,

$$
\left|u_{\nu}^{(k)}\right| \leq \sum_{\theta \in \Theta_{k, \nu}}|\operatorname{Val}(\theta)| \leq \Lambda_{1}^{\prime} \Lambda_{2}^{k} \Lambda_{3}^{k} e^{-\kappa|\boldsymbol{\nu}| / 4}
$$

what would imply in the convergence of expansion (2.12) provided $|\varepsilon|<\left(\Lambda_{2} \Lambda_{3}\right)^{-1}$.

It is clear that the above result is false since we cannot simply forget the self-energy graphs. The estimate obtained just illustrates the fact that all the problem concerning the convergence of the series (2.12) lies in the existence of self-energy graphs (small divisors). We have to overcome this difficult with some different approach.

\subsection{Renormalized expansion}

The problem with the self-energy graphs can be solved by a suitable resummation procedure of the formal series obtained from the coefficients (5.3). The basic idea is to "dress" the propagators $g_{\ell}^{\left(n_{\ell}\right)}$ in such a way that they could harbour all the malign contribution deriving from the self-energy graphs. The next step is to define an expansion in terms of only non-self-energy graphs and renormalized propagators which we hope to give an estimate like (5.5). This is something analogous to the procedure of mass renormalization in field theories. We shall therefore iteratively define new propagators $g_{\ell}^{\left[n_{\ell}\right]}$ (renormalized propagators).

Definition 5.6 (Self-Energy Value) Suppose that the renormalized propagators $g_{\ell}^{\left[n_{\ell}\right]}$ are given. For a self-energy graph $T$ which does not contain any other self-energy graph, we define the self-energy value associated with $T$ as

$$
\mathcal{V}_{T}(\boldsymbol{\omega} \cdot \boldsymbol{\nu} ; \varepsilon):=\varepsilon^{k_{T}}\left(\prod_{\ell \in L(T)} g_{\ell}^{\left[n_{\ell}\right]}\right)\left(\prod_{v \in B(T)} F_{v}\right)
$$

where $\boldsymbol{\nu}$ is the momentum which enters $T$ through the external line $\ell_{T}^{\mathrm{in}}, k_{T}=|B(T)|$, and $F_{v}$ is defined as in (4.2). Note that $\mathcal{V}_{T}(\boldsymbol{\omega} \cdot \boldsymbol{\nu} ; \varepsilon)$ depends on $\boldsymbol{\omega} \cdot \boldsymbol{\nu}$ through the propagators in $L(T)$. where

By setting $x=\boldsymbol{\omega} \cdot \boldsymbol{\nu}$ in (5.9) and $x_{\ell}=\boldsymbol{\omega} \cdot \boldsymbol{\nu}_{\ell}$ for each line $\ell \in L(T)$, one can write $x_{\ell}=x_{\ell}^{0}+\sigma_{\ell} x$,

$$
x_{\ell}^{0}=\boldsymbol{\omega} \cdot \boldsymbol{\nu}_{\ell}^{0}, \quad \boldsymbol{\nu}_{\ell}^{0}=\sum_{\substack{w \in B(T) \\ w \preceq v: \ell=\ell_{v}}} \boldsymbol{\nu}_{w},
$$

and $\sigma_{\ell}=1$ if $\ell$ is along the path of lines connecting the external lines of the self-energy $T$, and $\sigma_{\ell}=0$ otherwise.

Remark 5.7 The value $\mathcal{V}_{T}(x ; \varepsilon)$ of a self-energy graph $T$ can depend on $x$ only if $k_{T} \geq 2$.

Definition 5.8 We define $\Theta_{k, \nu}^{\mathcal{R}}$ as the set of renormalized trees, that is of trees which do not contain any self-energy graph. We also define $\mathcal{S}_{k, n}^{\mathcal{R}}$ as the set of self-energy graphs of order $k$ which do not contain any other self-energy graph and such that the maximum of scales of the lines in $T \in \mathcal{S}_{k, n}^{\mathcal{R}}$ is exactly $n$, and we call them the self-energy renormalized graphs of order $k$ and on scale $n$. We stress that the propagators associated with the lines in $\Theta_{k, \nu}^{\mathcal{R}}$ and $\mathcal{S}_{k, n}^{\mathcal{R}}$ are the renormalized ones, $g_{\ell}^{\left[n_{\ell}\right]}$. 
Then we can define the renormalized propagators $g_{\ell}^{[n]}:=g^{[n]}\left(\boldsymbol{\omega} \cdot \boldsymbol{\nu}_{\ell} ; \varepsilon\right)$ and the quantities $M^{[n]}(\boldsymbol{\omega}$. $\left.\boldsymbol{\nu}_{\ell} ; \varepsilon\right)$ recursively as follows. For $n_{0} \in \mathbb{Z}_{+}$we set

$$
\begin{aligned}
M^{\left[n_{0}-1\right]}(x ; \varepsilon) & :=0, \\
g^{\left[n_{0}\right]}(x ; \varepsilon) & :=\frac{\psi_{n_{0}}(|x|)}{i x}, \\
M^{\left[n_{0}\right]}(x ; \varepsilon) & :=\sum_{k=1}^{\infty} \sum_{T \in \mathcal{S}_{k, n_{0}}^{\mathcal{R}}} \mathcal{V}_{T}(x ; \varepsilon), \\
\mathcal{M}^{\left[n_{0}\right]}(x ; \varepsilon) & :=\chi_{n_{0}}(|x|) M^{\left[n_{0}\right]}(x ; \varepsilon),
\end{aligned}
$$

while, for $n \geq n_{0}+1$, by writing

$$
\begin{aligned}
& \Xi_{n}(x ; \varepsilon):=\chi_{n_{0}}(|x|) \ldots \chi_{n-1}\left(\left|i x-\mathcal{M}^{[n-2]}(x ; \varepsilon)\right|\right) \chi_{n}\left(\left|i x-\mathcal{M}^{[n-1]}(x ; \varepsilon)\right|\right), \\
& \Psi_{n}(x ; \varepsilon):=\chi_{n_{0}}(|x|) \ldots \chi_{n-1}\left(\left|i x-\mathcal{M}^{[n-2]}(x ; \varepsilon)\right|\right) \psi_{n}\left(\left|i x-\mathcal{M}^{[n-1]}(x ; \varepsilon)\right|\right),
\end{aligned}
$$

we define

$$
\begin{aligned}
g^{[n]}(x ; \varepsilon) & :=\frac{\Psi_{n}(x ; \varepsilon)}{i x-\mathcal{M}^{[n-1]}(x ; \varepsilon)}, \\
M^{[n]}(x ; \varepsilon) & :=\sum_{k=1}^{\infty} \sum_{T \in \mathcal{S}_{k, n}^{\mathcal{R}}} \mathcal{V}_{T}(x ; \varepsilon), \\
\mathcal{M}^{[n]}(x ; \varepsilon) & :=\mathcal{M}^{[n-1]}(x ; \varepsilon)+\Xi_{n}(x ; \varepsilon) M^{[n]}(x ; \varepsilon) \\
& =\sum_{j=n_{0}}^{n} \Xi_{j}(x ; \varepsilon) M^{[j]}(x ; \varepsilon),
\end{aligned}
$$

where $\mathcal{V}_{T}(x ; \varepsilon)$ is defined as in (5.9).

One should now realize, from the above definitions, that if $\theta$ is a tree in $\Theta_{k, \boldsymbol{\nu}}^{\mathcal{R}}$ or $\mathcal{S}_{k, n}^{\mathcal{R}}$, all of its lines are on scale $\geq n_{0}$. In particular, if $T \in \mathcal{S}_{k, n_{0}}^{\mathcal{R}}$, all lines in $T$ are exactly on the scale $n_{0}$ and, hence, for all $\ell \in L(T)$, the propagators are $g^{\left[n_{0}\right]}\left(x_{\ell} ; \varepsilon\right)$, as in (5.11).

Remark 5.9 Note that if a line $\ell$ is on scale $n \geq n_{0}+1$ and, by setting $x=\boldsymbol{\omega} \cdot \boldsymbol{\nu}_{\ell}$, one has $g^{[n]}(x ; \varepsilon) \neq 0$, this requires $\chi_{n_{0}}(|x|) \neq 0, \chi_{n_{0}+1}\left(\left|i x-\mathcal{M}^{\left[n_{0}\right]}(x ; \varepsilon)\right|\right) \neq 0, \ldots, \chi_{n-1}\left(\left|i x-\mathcal{M}^{[n-2]}(x ; \varepsilon)\right|\right) \neq 0$ and $\psi_{n}\left(\left|i x-\mathcal{M}^{[n-1]}(x ; \varepsilon)\right|\right) \neq 0$, which means

$$
\begin{aligned}
& |x| \leq 2^{-n_{0}} C_{1}, \\
& \left|i x-\mathcal{M}^{\left[n_{0}\right]}(x ; \varepsilon)\right| \leq 2^{-\left(n_{0}+1\right)} C_{1}, \\
& \left|i x-\mathcal{M}^{\left[n_{0}+1\right]}(x ; \varepsilon)\right| \leq 2^{-\left(n_{0}+2\right)} C_{1}, \\
& \ldots \ldots \ldots \\
& \left|i x-\mathcal{M}^{[n-2]}(x ; \varepsilon)\right| \leq 2^{-(n-1)} C_{1}, \\
& \left|i x-\mathcal{M}^{[n-1]}(x ; \varepsilon)\right| \geq 2^{-(n+1)} C_{1},
\end{aligned}
$$

so that, in particular, one has $\left|g_{\ell}^{[n]}\right| \leq C_{1}^{-1} 2^{n+1}$. If $\ell$ is on scale $n_{0}$ and $g^{\left[n_{0}\right]}(x ; \varepsilon) \neq 0$, then $\psi_{n_{0}}(|x|) \neq$ 0 , which implies that $\left|g_{\ell}^{\left[n_{0}\right]}\right| \leq C_{1}^{-1} 2^{n_{0}+1}$.

Then we define, formally, for $\boldsymbol{\nu} \neq \mathbf{0}$,

$$
u_{\nu}^{[k]}=\sum_{\theta \in \Theta_{k, \nu}^{\mathcal{R}}} \operatorname{Val}(\theta), \quad \operatorname{Val}(\theta)=\left(\prod_{\ell \in L(\theta)} g_{\ell}^{\left[n_{\ell}\right]}\right)\left(\prod_{v \in B(\theta)} F_{v}\right),
$$


while, for $\boldsymbol{\nu}=\mathbf{0}$, one has $u_{\mathbf{0}}^{[k]}=0$, and we write

$$
\bar{u}(t)=\sum_{k=0}^{\infty} \varepsilon^{k} u^{[k]}(t)=\sum_{k=1}^{\infty} \varepsilon^{k} \sum_{\boldsymbol{\nu} \in \mathbb{Z}^{d}} e^{i \boldsymbol{\nu} \cdot \boldsymbol{\omega} t} u_{\boldsymbol{\nu}}^{[k]}
$$

where the coefficients $u_{\nu}^{[k]}$ depend on $\varepsilon$ (as the propagators do); note that the order $k$ of a renormalized tree $\theta$ is still defined as $k=|B(\theta)|$, but it does not correspond to the perturbative order any more.

Definition 5.10 Let $\boldsymbol{\omega}$ satisfy the Diophantine conditions 2.14). Fix $\varepsilon$ such that one has

$$
\left|i \boldsymbol{\omega} \cdot \boldsymbol{\nu}-\mathcal{M}^{[n]}(\boldsymbol{\omega} \cdot \boldsymbol{\nu} ; \varepsilon)\right| \geq C_{1}|\boldsymbol{\nu}|^{-\tau_{1}} \quad \forall \boldsymbol{\nu} \in \mathbb{Z}_{*}^{d} \text { and } \forall n \geq n_{0},
$$

with Diophantine constants $C_{1}$ and $\tau_{1}$, where $\tau_{1}>\tau$ and $C_{1}<C_{0}$ are to be fixed later. We call $\mathcal{E}_{*}$ the set of $\varepsilon$ for which the Diophantine conditions (5.15) are satisfied, and we shall refer to it as the set of admissible values of $\varepsilon$.

We shall see in next section that for $\varepsilon \in \mathcal{E}_{*}$ we shall be able to give a meaning to the (so far formal) renormalized expansion (5.14), hence we shall prove that the set $\mathcal{E}_{*}$ has positive Lebesgue measure, provided that $\tau_{1}$ and $C_{1}^{-1}$ are chosen large enough.

Fix $\bar{\varepsilon}$ such that the series obtained from (5.14) by replacing $g_{\ell}^{\left[n_{\ell}\right]}$ in (5.13) with the bound $2^{n_{\ell}+1} C_{1}^{-1}$ converges for $|\varepsilon| \leq \bar{\varepsilon}$, and fix $\varepsilon_{0} \leq \bar{\varepsilon}$ small enough (how small will be determined by the forthcoming analysis). In the following we shall consider the interval $\left[0, \varepsilon_{0}\right]$; the interval $\left[-\varepsilon_{0}, 0\right]$ can be studied in the same way.

It will be convenient to split the interval $\left[0, \varepsilon_{0}\right]$ into infinitely many disjoint intervals by setting

$$
\left[0, \varepsilon_{0}\right]=\{0\} \cup \bigcup_{m=0}^{\infty} \mathcal{E}_{m}, \quad \mathcal{E}_{m}:=\left(2^{-(m+1)} \varepsilon_{0}, 2^{-m} \varepsilon_{0}\right],
$$

and to study separately each interval $\mathcal{E}_{m}$. We shall prove that for each $m$ the admissible values of $\varepsilon$ inside $\mathcal{E}_{m}$ have large measure, and that the their relative measure meas $\left(\mathcal{E}_{m} \cap \mathcal{E}_{*}\right) / \operatorname{meas}\left(\mathcal{E}_{m}\right)$ tends to 1 as $m$ tends to infinity.

Therefore in the following we imagine we have fixed $m$, and we set $\varepsilon_{m}=2^{-m} \varepsilon_{0}$, so that we can write $\mathcal{E}_{m}=\left(\varepsilon_{m} / 2, \varepsilon_{m}\right]$.

\section{Properties of the self-energy values}

Given a self-energy $T \in \mathcal{S}_{k, n_{0}}^{\mathcal{R}}$ define

$$
\overline{\mathcal{V}}_{T}(x ; \varepsilon):=\varepsilon^{k_{T}}\left(\prod_{\ell \in L(T)} \frac{1}{i x_{\ell}}\right)\left(\prod_{v \in B(T)} F_{v}\right),
$$

which differs from $\mathcal{V}_{T}(x ; \varepsilon)$ as $\psi_{n_{0}}\left(\left|x_{\ell}\right|\right)$ is replaced with 1 for all $\ell \in L(T)$, and set

$$
\begin{array}{ll}
\mathcal{M}_{j}^{\left[n_{0}\right]}(x ; \varepsilon)=\chi_{n_{0}}(|x|) M_{j}^{\left[n_{0}\right]}(x ; \varepsilon), & M_{j}^{\left[n_{0}\right]}(x ; \varepsilon)=\sum_{k=1}^{j} \varepsilon^{k} \sum_{T \in \mathcal{S}_{k, n_{0}}^{\mathcal{R}}} \mathcal{V}_{T}(x ; \varepsilon), \\
\overline{\mathcal{M}}_{j}^{\left[n_{0}\right]}(x ; \varepsilon)=\chi_{n_{0}}(|x|) \bar{M}_{j}^{\left[n_{0}\right]}(x ; \varepsilon), & \bar{M}_{j}^{\left[n_{0}\right]}(x ; \varepsilon)=\sum_{k=1}^{j} \varepsilon^{k} \sum_{T \in \mathcal{S}_{k, n_{0}}^{\mathcal{R}}} \overline{\mathcal{V}}_{T}(x ; \varepsilon) .
\end{array}
$$


This allows us to decompose

$$
\mathcal{M}_{j}^{\left[n_{0}\right]}(0 ; \varepsilon)=\overline{\mathcal{M}}_{j}^{\left[n_{0}\right]}(0 ; \varepsilon)+\left(\mathcal{M}_{j}^{\left[n_{0}\right]}(0 ; \varepsilon)-\overline{\mathcal{M}}_{j}^{\left[n_{0}\right]}(0 ; \varepsilon)\right) .
$$

where $\overline{\mathcal{M}}_{j}^{\left[n_{0}\right]}(0 ; \varepsilon)$ depends neither on $x$ nor on $n_{0}$. Note that one has $\mathcal{M}_{j}^{\left[n_{0}\right]}(0 ; \varepsilon)=M_{j}^{\left[n_{0}\right]}(0 ; \varepsilon)$ and $\overline{\mathcal{M}}_{j}^{\left[n_{0}\right]}(0 ; \varepsilon)=\bar{M}_{j}^{\left[n_{0}\right]}(0 ; \varepsilon)$ as $\chi_{n_{0}}(0)=1$ for all $n_{0} \geq 0$.

Lemma 6.1 Let $G_{j}, j \geq 1$, be as the previous sections. Then one has

$$
\overline{\mathcal{M}}_{j_{0}}^{\left[n_{0}\right]}(0 ; \varepsilon)=\sum_{k=1}^{j_{0}} \varepsilon^{k} G_{k}
$$

for all $n_{0}$ and all $j_{0}$.

Proof. By setting $x=0$ any self-energy graph $T$ in $\mathcal{S}_{k, n_{0}}^{\mathcal{R}}$ contributing to $\overline{\mathcal{M}}_{j_{0}}^{\left[n_{0}\right]}(x ; \varepsilon)$ looks like a tree $\theta$ in $\widetilde{\mathcal{T}}_{k, \mathbf{0}}$, except for the presence of the scale labels (compare (6.1) with (5.9): if $T \in \mathcal{S}_{k, n_{0}}^{\mathcal{R}}$ each line $\ell \in L(T)$ has scale $n_{\ell}=n_{0}$ ). Nevertheless the corresponding propagators do not depend on the scales. Hence

$$
\sum_{T \in \mathcal{S}_{k, n}^{\mathcal{R}}} \overline{\mathcal{V}}_{T}(0 ; \varepsilon)=\sum_{\theta \in \widetilde{\mathcal{T}}_{k, 0}} \operatorname{Val}(\theta),
$$

so that the assertion follows from the definition of $G_{k}$ (see Lemma 3.5).

Lemma 6.2 For any self-energy $T$ one has

$$
1-\prod_{\ell \in L(T)}\left(1-\chi_{n_{0}}\left(\left|x_{\ell}\right|\right)\right) \leq \sum_{\ell \in L(T)} \chi_{n_{0}}\left(\left|x_{\ell}\right|\right),
$$

and the same result holds if each $x_{\ell}$ is replaced with $x_{\ell}^{0}$.

Proof. It follows from the identity

$$
\prod_{j=1}^{n}\left(1-a_{j}\right)=1-a_{1}-\sum_{j=2}^{n} a_{j} \prod_{i=1}^{j-1}\left(1-a_{i}\right)
$$

with $n \geq 2$ and $0 \leq a_{j} \leq 1$, which can be easily proved by induction.

Lemma 6.3 For all $n_{0}$ one has

$$
\begin{aligned}
\left|\mathcal{M}_{j_{0}}^{\left[n_{0}\right]}(0 ; \varepsilon)-\overline{\mathcal{M}}_{j_{0}}^{\left[n_{0}\right]}(0 ; \varepsilon)\right| & \leq B_{1}|\varepsilon| e^{-B_{2} 2^{n_{0} / \tau_{1}}}, \\
\left|\partial_{\varepsilon}\left(\mathcal{M}_{j_{0}}^{\left[n_{0}\right]}(0 ; \varepsilon)-\overline{\mathcal{M}}_{j_{0}}^{\left[n_{0}\right]}(0 ; \varepsilon)\right)\right| & \leq B_{1} e^{-B_{2} 2^{n_{0} / \tau_{1}}},
\end{aligned}
$$

for suitable constants $B_{1}$ and $B_{2}$, depending on $j_{0}$ but independent of $n_{0}$.

Proof. One can write $M^{\left[n_{0}\right]}(0 ; \varepsilon)$ as in (5.11) with $x=0$, where $\mathcal{V}_{T}(0 ; \varepsilon)$ is given by (5.9) with $n_{\ell}=n_{0}$ and

$$
g_{\ell}^{\left[n_{\ell}\right]}=\frac{\psi_{n_{0}}\left(\left|x_{\ell}^{0}\right|\right)}{i x_{\ell}^{0}}=\frac{1-\chi_{n_{0}}\left(\left|x_{\ell}^{0}\right|\right)}{i x_{\ell}^{0}} .
$$

Furthermore $\mathcal{M}_{j_{0}}^{\left[n_{0}\right]}(x ; \varepsilon)$ and $\overline{\mathcal{M}}_{j_{0}}^{\left[n_{0}\right]}(0 ; \varepsilon)$ are polynomials of degree $j_{0}$ in $\varepsilon$, hence one has

$$
\mathcal{M}_{j_{0}}^{\left[n_{0}\right]}(0 ; \varepsilon)-\overline{\mathcal{M}}_{j_{0}}^{\left[n_{0}\right]}(0 ; \varepsilon)=\sum_{k=1}^{j_{0}} \sum_{T \in \mathcal{S}_{k, n_{0}}^{\mathcal{R}}}\left(\mathcal{V}_{T}(0 ; \varepsilon)-\overline{\mathcal{V}}_{T}(0 ; \varepsilon)\right)
$$


which is trivially differentiable with respect to $\varepsilon$. By applying Lemma 6.2 we obtain

$$
\begin{aligned}
& \left|\left(\mathcal{M}_{j_{0}}^{\left[n_{0}\right]}(0 ; \varepsilon)-\overline{\mathcal{M}}_{j_{0}}^{\left[n_{0}\right]}(0 ; \varepsilon)\right)\right| \\
& \quad \leq \sum_{k=1}^{j_{0}}|\varepsilon|^{k} \sum_{T \in \mathcal{S}_{k, n_{0}}^{\mathcal{R}}} \sum_{\ell^{\prime} \in L(T)} \chi_{n_{0}}\left(\left|x_{\ell^{\prime}}^{0}\right|\right)\left(\prod_{\ell \in L(T)} \frac{1}{\left|x_{\ell}^{0}\right|}\right)\left(\prod_{v \in B(T)}\left|F_{v}\right|\right),
\end{aligned}
$$

and for $\partial_{\varepsilon}\left(\mathcal{M}_{j_{0}}^{\left[n_{0}\right]}(0 ; \varepsilon)-\overline{\mathcal{M}}_{j_{0}}^{\left[n_{0}\right]}(0 ; \varepsilon)\right)$ the same bound can be obtained with $k|\varepsilon|^{k-1}$ instead of $|\varepsilon|^{k}$. In (6.5) the factor $\chi_{n_{0}}\left(\left|x_{\ell^{\prime}}^{0}\right|\right)$ requires $\left|x_{\ell^{\prime}}^{0}\right| \leq C_{1} 2^{-n_{0}}$, so that by the Diophantine condition (2.14) one has $\left|\boldsymbol{\nu}_{\ell^{\prime}}^{0}\right| \geq 2^{n_{0} / \tau}$, hence in

$$
\left(\prod_{v \in B(T)}\left|F_{v}\right|\right) \leq \mathcal{Q}^{|B(T)|}\left(\prod_{v \in B(T)} e^{-\kappa\left|\boldsymbol{\nu}_{v}\right| / 2}\right)\left(\prod_{v \in B(T)} e^{-\kappa\left|\boldsymbol{\nu}_{v}\right| / 4}\right)\left(\prod_{v \in B(T)} e^{-\kappa\left|\boldsymbol{\nu}_{v}\right| / 4}\right)
$$

one can bound the third product by

$$
\left(\prod_{v \in B(T)} e^{-\kappa\left|\boldsymbol{\nu}_{v}\right| / 4}\right) \leq e^{-\kappa \mid \boldsymbol{\nu}_{\ell^{\prime}}^{0} / / 4} \leq e^{-\kappa 2^{n_{0} / \tau} / 4}<e^{-\kappa 2^{n_{0} / \tau_{1}} / 4},
$$

if $\tau_{1}>\tau_{0}$, while using the second product to perform the sum over the mode labels and the first one to find, by reasoning as for (4.3) to (4.5),

$$
\left(\prod_{\ell \in L(T)} \frac{1}{\left|x_{\ell}^{0}\right|}\right)\left(\prod_{v \in B(T)} e^{-\kappa\left|\boldsymbol{\nu}_{v}\right| / 2}\right) \leq\left(\frac{\tau !}{C_{0}}\left(\frac{2|L(T)|}{\kappa}\right)^{\tau}\right)^{|L(T)|} \leq \bar{\Gamma}_{1} \bar{\Gamma}_{2}^{k_{T}}\left(k_{T} !\right)^{\beta},
$$

with $k_{T}=k$ for $T \in \mathcal{S}_{k, n_{0}}^{\mathcal{R}}$, so that, by collecting together the bounds and inserting them into 6.5. we prove the assertion. In particular $B_{1}$ is proportional to $\bar{\Gamma}_{2}^{j_{0}}\left(j_{0} !\right)^{\beta}$, while $B_{2}$ is independent of $j_{0}$.

Lemma 6.4 Let $G_{j}, j \geq 1$, be as the previous sections. Assume that there is $j_{0} \in \mathbb{N}$ such that $G_{j_{0}} \neq 0$ and $G_{j}=0$ for all $1 \leq j<j_{0}$. There exists two constants $c_{1}$ and $c_{2}$, depending on $j_{0}$, such that for

$$
n_{0} \geq \tau_{1} \log _{2}\left(c_{1}+c_{2} \log \frac{1}{|\varepsilon|}\right)
$$

one has

$$
\left|\partial_{\varepsilon} \mathcal{M}_{j_{0}}^{\left[n_{0}\right]}(0 ; \varepsilon)\right| \geq \frac{j_{0}}{2}|\varepsilon|^{j_{0}-1}\left|G_{j_{0}}\right| .
$$

provided $\varepsilon$ is small enough. If $j_{0}=1$ one can take $c_{2}=0$.

Proof. One can write

$$
\overline{\mathcal{M}}_{j_{0}}^{\left[n_{0}\right]}(0 ; \varepsilon)=\sum_{k=1}^{j_{0}} \varepsilon^{k} G_{k}=\varepsilon^{j_{0}} G_{j_{0}}
$$

by Lemma 6.1 so that

$$
\partial_{\varepsilon} \overline{\mathcal{M}}_{j_{0}}^{\left[n_{0}\right]}(0 ; \varepsilon)=j_{0} \varepsilon^{j_{0}-1} G_{j_{0}}
$$

By Lemma 6.3 we can bound

$$
\left|\partial_{\varepsilon}\left(\mathcal{M}_{j_{0}}^{\left[n_{0}\right]}(0 ; \varepsilon)-\overline{\mathcal{M}}_{j_{0}}^{\left[n_{0}\right]}(0 ; \varepsilon)\right)\right| \leq \beta_{1} B_{1} e^{-\beta_{2} B_{2} 2^{n_{0} / \tau_{1}}} \leq \frac{1}{2}|\varepsilon|^{j_{0}-1}\left|G_{j_{0}}\right|,
$$


where the first inequality is obtained as soon as $\beta_{2} \leq 1$ and $\beta_{1} \geq 1$, while the second one requires

$$
n_{0} \geq \tau_{1} \log _{2}\left(\frac{1}{\beta_{2} B_{2}} \log \frac{2 \beta_{1} B_{1}}{\left|G_{j_{0}}\right|}+\frac{j_{0}-1}{\beta_{2} B_{2}} \log \frac{1}{|\varepsilon|}\right),
$$

so that the assertion follows if $c_{1}$ and $c_{2}$ are chosen according to (6.11).

Remark 6.5 The constants $\beta_{1}$ and $\beta_{2}$ in (6.10) could be taken $\beta_{1}=\beta_{2}=1$. However in the following it will turn out useful to have some freedom in fixing their values; see in particular Remark 7.6 .

Remark 6.6 Note that if we choose $n_{0}=\tau_{1} \log _{2}\left(c_{1}+c_{2} \log \left(2 / \varepsilon_{m}\right)\right)$ we obtain a value of $n_{0}$ which can be used for all $\varepsilon \in \mathcal{E}_{m}$.

\section{Convergence of the renormalized expansion}

We are left with the problem of proving that the series defining the renormalized expansion (5.14) converges, and of studying how large is the set $\mathcal{E}_{*} \cap\left[0, \varepsilon_{0}\right]$ of admissible values of $\varepsilon$; we shall verify that it is a set with positive relatively large measure.

As we have fixed $m$, for notational simplicity, in the following we shall find convenient to shorthand $\mathcal{E}^{[\infty]} \equiv \mathcal{E}_{*} \cap \mathcal{E}_{m}$. We shall assume $\varepsilon \in \mathcal{E}^{[\infty]}$, and $n_{0}$ fixed as in Remark [6.6]

Lemma 7.1 Assume that the set $\mathcal{E}^{[\infty]}$ has non-zero measure and that for all $\varepsilon \in \mathcal{E}^{[\infty]}$ and for all $n_{0} \leq j<n-1$ the functions $\mathcal{M}^{[j]}(x ; \varepsilon)$ are $C^{1}$ in $x$ and satisfy the bounds

$$
\left|\mathcal{M}^{[j]}(x ; \varepsilon)\right| \leq D \sqrt{|\varepsilon|}, \quad\left|\partial_{x} \mathcal{M}^{[j]}(x ; \varepsilon)\right| \leq D \sqrt{|\varepsilon|},
$$

for some constant $D$. There there exists a positive constant $c$, independent of $n$, such that for any renormalized tree $\theta$ with $\operatorname{Val}(\theta) \neq 0$ the number $N_{j}(\theta)$ of lines on scale $j$ satisfies the bound

$$
N_{j}(\theta) \leq c 2^{-j / \tau_{1}} \sum_{v \in B(\theta)}\left|\boldsymbol{\nu}_{v}\right|
$$

for all $n_{0}<j \leq n-1$.

Proof. Set $K(\theta):=\sum_{v \in B(\theta)}\left|\boldsymbol{\nu}_{v}\right|$ and call $N_{j}^{\dagger}(\theta)$ the number of lines in $L(\theta)$ on scale $j^{\prime} \geq j$. We prove inductively on the order $k$ of the renormalized trees the bound

$$
N_{j}^{\dagger}(\theta) \leq \max \left\{0,2 K(\theta) 2^{(3-j) / \tau_{1}}-1\right\}
$$

for all $n_{0}<j \leq n-1$

If $\theta$ has $k=0$ one has $B(\theta)=\{v\}$ and $K(\theta)=\left|\boldsymbol{\nu}_{v}\right|$. The line $\ell$ exiting $v$ can be on scale $n_{\ell} \geq j$ only if have $\left|i x-\mathcal{M}^{[j-2]}(x ; \varepsilon)\right| \leq 2^{-j+1} C_{1}$ (see Remark [5.9), with $x=\boldsymbol{\omega} \cdot \boldsymbol{\nu}_{v}$, hence, by the Diophantine conditions (5.15), one has $\left|\boldsymbol{\nu}_{v}\right| \geq 2^{(j-1) / \tau_{1}}$, which implies $2 K(\theta) 2^{(3-j) / \tau_{1}} \geq 22^{2 / \tau_{1}} \geq 2$. Therefore in such a case the bound (7.3) is trivially satisfied.

If $\theta$ is a renormalized tree of order $k \geq 1$, we assume that the bound holds for all renormalized trees of order $k^{\prime}<k$. Define $E_{j}=\left(22^{(3-j) / \tau_{1}}\right)^{-1}$ : so we have to prove that $N_{j}^{\dagger}(\theta) \leq \max \left\{0, K(\theta) E_{j}^{-1}-1\right\}$.

Call $\ell$ the root line of $\theta$ and $\ell_{1}, \ldots, \ell_{m}$ the $m \geq 0$ lines on scales $\geq j$ which are the closest to $\ell$ (i.e. such that no other line along the paths connecting the lines $\ell_{1}, \ldots, \ell_{m}$ to the root line is on scale $\geq j$ ).

If the root line $\ell$ of $\theta$ is on scale $n_{\ell}<j$, then

$$
N_{j}^{\dagger}(\theta)=\sum_{i=1}^{m} N_{j}^{\dagger}\left(\theta_{i}\right),
$$


where $\theta_{i}$ is the renormalized subtree with $\ell_{i}$ as root line, hence the bound follows by the inductive hypothesis.

If the root line $\ell$ has scale $n_{\ell} \geq j$, then $\ell_{1}, \ldots, \ell_{m}$ are the entering lines of a cluster $T$.

By denoting again with $\theta_{i}$ the renormalized subtree having $\ell_{i}$ as root line, one has

$$
N_{j}^{\dagger}(\theta)=1+\sum_{i=1}^{m} N_{j}^{\dagger}\left(\theta_{i}\right)
$$

so that the bound becomes trivial if either $m=0$ or $m \geq 2$.

If $m=1$ then one has a cluster $T$ with two external lines $\ell$ and $\ell_{1}$, which are both with scales $\geq j$. Set $x_{\ell}=\boldsymbol{\omega} \cdot \boldsymbol{\nu}_{\ell}$ and $x_{\ell_{1}}=\boldsymbol{\omega} \cdot \boldsymbol{\nu}_{\ell_{1}}$; then

$$
\left|i x_{\ell}-\mathcal{M}^{[j-2]}\left(x_{\ell} ; \varepsilon\right)\right| \leq 2^{-j+1} C_{1}, \quad\left|i x_{\ell_{1}}-\mathcal{M}^{[j-2]}\left(x_{\ell_{1}} ; \varepsilon\right)\right| \leq 2^{-j+1} C_{1},
$$

and $\boldsymbol{\nu}_{\ell} \neq \boldsymbol{\nu}_{\ell_{1}}$, otherwise $T$ would be a self-energy graph. Then, by (7.5), one has

$$
\begin{aligned}
2^{-j+2} C_{1} & \geq\left|i\left(x_{\ell}-x_{\ell_{1}}\right)-\mathcal{M}^{[j-2]}\left(x_{\ell} ; \varepsilon\right)+\mathcal{M}^{[j-2]}\left(x_{\ell_{1}} ; \varepsilon\right)\right| \\
& =\left|\left(x_{\ell}-x_{\ell_{1}}\right)\left(i-\partial_{x} \mathcal{M}^{[j-2]}\left(x_{*} ; \varepsilon\right)\right)\right| \\
& \geq\left|x_{\ell}-x_{\ell_{1}}\right|(1-D \sqrt{|\varepsilon|}) \geq \frac{1}{2}\left|x_{\ell}-x_{\ell_{1}}\right|,
\end{aligned}
$$

where $x_{*}$ is a point between $x_{\ell}$ and $x_{\ell_{1}}$, and the assumption (7.1) has been used. Hence by the Diophantine condition (2.14), one has $\left|\boldsymbol{\nu}_{\ell}-\boldsymbol{\nu}_{\ell_{1}}\right|>2^{(j-3) / \tau_{1}}$, so that

$$
\sum_{v \in B(T)}\left|\boldsymbol{\nu}_{v}\right| \geq\left|\boldsymbol{\nu}_{T}\right|=\left|\boldsymbol{\nu}_{\ell}-\boldsymbol{\nu}_{\ell_{1}}\right|>2^{(j-3) / \tau_{1}}>E_{j},
$$

hence $K(\theta)-K\left(\theta_{1}\right)>E_{j}$, which, inserted into (7.4) with $m=1$, gives, by using the inductive hypothesis,

$$
\begin{aligned}
N_{j}^{\dagger}(\theta) & =1+N_{j}^{\dagger}\left(\theta_{1}\right) \leq 1+K\left(\theta_{1}\right) E_{j}^{-1}-1 \\
& \leq 1+\left(K(\theta)-E_{j}\right) E_{j}^{-1}-1 \leq K(\theta) E_{j}^{-1}-1,
\end{aligned}
$$

hence the bound is proved also if the root line is on scale $n_{\ell} \geq j$.

Remark 7.2 Let $j_{0}$ be as in Lemma 6.4. If $\varepsilon_{0}$ is small enough, for all $\varepsilon \in\left(\varepsilon_{m} / 2, \varepsilon_{m}\right]$ and $n_{0}$ chosen according to Remark [6.6 if $j_{0}=1$ we can bound

$$
|\varepsilon|^{k} 2^{(2 k-1) n_{0}} \leq c_{1}^{(2 k-1) \tau_{1}}|\varepsilon|^{k},
$$

while if $j_{0}>1$ we can bound

$$
|\varepsilon|^{k} 2^{(2 k-1) n_{0}} \leq\left(2 c_{2}\right)^{(2 k-1) \tau_{1}}|\varepsilon|^{k}\left(\log \frac{2}{|\varepsilon|}\right)^{(2 k-1) \tau_{1}},
$$

where, under the same smallness assumption on $\varepsilon$, one has

$$
\left(\log \frac{2}{|\varepsilon|}\right)^{\tau_{1}} \leq S_{p}\left(\frac{1}{|\varepsilon|}\right)^{p}
$$

for all $p>0$ and with $S_{p}$ a positive constant depending on $p$. Hence, in 7.7), by taking $p \leq 1 / 4$, one obtains for $j_{0}>1$

$$
|\varepsilon|^{k} 2^{n_{0}(2 k-1)} \leq\left(2 c_{2}\right)^{(2 k-1) \tau_{1}} S_{p}^{2 k-1}|\varepsilon|^{k / 2} .
$$


Therefore, whichever the value of $j_{0}$ is, we can bound

$$
|\varepsilon|^{k} 2^{(2 k-1) n_{0}} \leq c_{3}|\varepsilon|^{k / 2},
$$

for all $k \geq 1$, with $c_{3}$ a suitable positive constant.

Remark 7.3 In particular one can choose $p \leq 1 /\left(2\left(2 j_{0}+1\right)\right)$, which implies

$$
|\varepsilon|^{k-1}\left(\log \frac{2}{|\varepsilon|}\right)^{(2 k-1) \tau_{1}} \leq|\varepsilon|^{j_{0}-1} \sqrt{|\varepsilon|}|\varepsilon|^{\left(k-j_{0}-1\right) / 2}
$$

for all $k \geq j_{0}+1$, a property which will be useful in the following.

Lemma 7.4 Fix $p$ as in Remark 7.2. Then one has

$$
\left|\mathcal{M}^{\left[n_{0}\right]}(x ; \varepsilon)\right| \leq D \sqrt{|\varepsilon|}, \quad\left|\partial_{x} \mathcal{M}^{\left[n_{0}\right]}(x ; \varepsilon)\right| \leq D|\varepsilon|,
$$

for suitable positive constants $D$ and $D^{\prime}$.

Proof. The first bound follows from (7.10).

Let $j_{0}$ be as in Lemma 6.4. If $j_{0}=1$ then $n_{0}$ does not depend on $\varepsilon$, and also the bound second is trivially satisfied.

If $j_{0} \geq 2$, in order to obtain the second bound, one can discuss in a different ways contributions with $k_{T}=1$ and contributions with $k_{T} \geq 2$. If $k_{T}=1$ then $\mathcal{V}_{T}(x ; \varepsilon)$ does not depend on $x$ (see Remark (5.7), so that, by using the notations (6.2), one has $\mathcal{M}_{1}^{\left[n_{0}\right]}(x ; \varepsilon)=\chi_{n_{0}}(|x|) M_{1}^{\left[n_{0}\right]}(0 ; \varepsilon)$, and one can write

$$
M_{1}^{\left[n_{0}\right]}(0 ; \varepsilon)=\bar{M}_{1}^{\left[n_{0}\right]}(0 ; \varepsilon)+\left(M_{1}^{\left[n_{0}\right]}(0 ; \varepsilon)-\bar{M}_{1}^{\left[n_{0}\right]}(0 ; \varepsilon)\right),
$$

where $\bar{M}_{1}^{\left[n_{0}\right]}(0 ; \varepsilon)=0$ by Lemma 6.1 (and the definition of $j_{0}$ ), while the difference

$$
\mathcal{M}_{1}^{\left[n_{0}\right]}(0 ; \varepsilon)-\overline{\mathcal{M}}_{1}^{\left[n_{0}\right]}(0 ; \varepsilon)=\chi_{n_{0}}(|x|)\left(M_{1}^{\left[n_{0}\right]}(0 ; \varepsilon)-\bar{M}_{1}^{\left[n_{0}\right]}(0 ; \varepsilon)\right)
$$

can be bounded through Lemma 6.3 proportionally to $e^{-B_{2} 2^{n_{0} / \tau_{1}}}$. Hence the derivative with respect to $x$ acts only on the compact support function $\chi_{n_{0}}(|x|)$ and produces a factor $2^{n_{0}}$ which is controlled by the exponentially small factor $e^{-B_{2} 2^{n_{0} / \tau_{1}}}$. The conclusion is that the contributions with $k_{T}=1$ can be bounded proportionally to $\varepsilon$. The contributions with $k_{T}=2$ can be bounded relying again on the bound (7.10).

Lemma 7.5 Fix $p$ as in Remark 7.2 and $n_{0}$ as in Remark [6.6. For $\varepsilon \in \mathcal{E}^{[\infty]}$ and for $x$ such that $g^{[n]}(x ; \varepsilon) \neq 0$, there exist two constants $D$ and $D^{\prime}$ such that the functions $\mathcal{M}^{[j]}(x ; \varepsilon)$ are smooth functions of $x$ and satisfy the bounds

$$
\begin{aligned}
& \left|\mathcal{M}^{[j]}(x ; \varepsilon)\right| \leq D \sqrt{|\varepsilon|}, \quad\left|\partial_{x} \mathcal{M}^{[j]}(x ; \varepsilon)\right| \leq D|\varepsilon|, \\
& \left|\mathcal{M}^{[j]}(x ; \varepsilon)-\mathcal{M}^{[j-1]}(x ; \varepsilon)\right| \leq D|\varepsilon| e^{-D^{\prime} 2^{j / \tau_{1}}},
\end{aligned}
$$

for all $n_{0}<j \leq n-1$. Furthermore for all $T$ contributing to $\mathcal{M}^{[j]}(x ; \varepsilon)$, with $n_{0}<j \leq n-1$, one has

$$
N_{j^{\prime}}(T) \leq c 2^{-j^{\prime} / \tau_{1}} \sum_{v \in B(T)}\left|\boldsymbol{\nu}_{v}\right|
$$

for all $j^{\prime} \leq j$. 
Proof. The first bound in (7.11) can be proved by induction on $n_{0} \leq j \leq n-1$. For $j=n_{0}$ it has been already checked (see Lemma [7.4). Let us assume that it holds for all $n_{0} \leq j^{\prime}<j$. One can proceed as for the proof of Lemma 2 in Ref. [13]. First of all one can prove for any self-energy graph $T \in \mathcal{S}_{k, j}^{\mathcal{R}}$ the inequalities

$$
\sum_{v \in B(T)}\left|\boldsymbol{\nu}_{v}\right|>2^{(j-4) / \tau_{1}}, \quad N_{j^{\prime}}(T) \leq 22^{\left(3-j^{\prime}\right) / \tau_{1}} \sum_{v \in B(T)}\left|\boldsymbol{\nu}_{v}\right|, \quad n_{0}+1 \leq j^{\prime} \leq j,
$$

where $N_{j^{\prime}}(T)$ denotes the number of lines on scales $j^{\prime}$ contained in $T$. We omit the proof, as it is identical to that given in Ref. [13].

The estimates (7.13) allow us to bound

$$
\left|\mathcal{V}_{T}(x ; \varepsilon)\right| \leq|\varepsilon|^{k} A_{1} A_{2}^{k} e^{-A_{3} 2^{j / \tau_{1}}} \prod_{v \in B(T)} e^{-\kappa\left|\boldsymbol{\nu}_{v}\right| / 2} .
$$

The only difference with respect to the analogous bound (7.18) in Ref. 13] is that the constants $A_{1}$ and $A_{2}$ depend on $\varepsilon$. In fact given a self-energy graph $T \in \mathcal{S}_{k, j}^{\mathcal{R}}$, if we express its value according to (5.9), we can bound

$$
\prod_{\ell \in L(T)}\left|g_{\ell}^{\left[n_{\ell}\right]}\right| \leq\left(2 C_{1}^{-1}\right)^{|L(T)|} 2^{n_{0} N_{n_{0}}(T)}\left(\prod_{n=n_{0}+1}^{j} 2^{n N_{n}(T)}\right),
$$

with $N_{n_{0}}(T) \leq 2 k-1$ and $N_{n}(T) \leq c 2^{-n / \tau_{1}} \sum_{v \in B(T)}\left|\boldsymbol{\nu}_{v}\right|$ for all $n_{0}+1 \leq n \leq j$, as it follows from the second bound in (7.13). Hence the last product can be bounded by using the bound on $N_{n}(T)$ and (5.7) with $n_{1}(\kappa, c, \tau)=n_{0}$ : just note that for $\varepsilon_{0}$ small enough such a choice for $n_{1}(\kappa, c, \tau)$ automatically satisfies the inequality in (5.7). Then we can apply the bounds given in Remark [7.2 to write

$$
|\varepsilon|^{k} A_{1} A_{2}^{k}=\bar{A}_{1} \bar{A}_{2}^{k}|\varepsilon|^{k / 2},
$$

with $\bar{A}_{1}$ and $\bar{A}_{2}$ two constants independent of $\varepsilon$. Then the first bound in (7.11) is proven.

To obtain the third bound in (7.11) we note that one has for $j \geq n_{0}+1$

$$
\begin{aligned}
\left|\mathcal{M}^{[j]}(x ; \varepsilon)-\mathcal{M}^{[j-1]}(x ; \varepsilon)\right| & \leq\left|M^{[j]}(x ; \varepsilon)\right| \leq \sum_{k=1}^{\infty} \sum_{T \in \mathcal{S}_{k, j}^{\mathcal{R}}}\left|\mathcal{V}_{T}(x ; \varepsilon)\right| \\
& =\sum_{T \in \mathcal{S}_{1, j}^{\mathcal{R}}}\left|\mathcal{V}_{T}(x ; \varepsilon)\right|+\sum_{k=2}^{\infty} \sum_{T \in \mathcal{S}_{k, j}^{\mathcal{R}}}\left|\mathcal{V}_{T}(x ; \varepsilon)\right|,
\end{aligned}
$$

where the last sum can be bounded proportionally to $|\varepsilon| e^{-A_{3} 2^{j / \tau_{1}}}$, because of (17.14) and (17.15). The first one can be bounded proportionally to $|\varepsilon|$ if $j_{0}=1$. If $j_{0} \geq 2$ we can reason as follows. We can bound $\left|\mathcal{V}_{T}(x ; \varepsilon)\right|$ according to $(\mathbf{7 . 1 4})$, with $k=1$, and write $e^{-A_{3} 2^{j / \tau_{1}}}=e^{-A_{3} 2^{j / \tau_{1}} / 2} e^{-A_{3} 2^{j / \tau_{1}} / 4} e^{-A_{3} 2^{j / \tau_{1}} / 4}$. The self-energy graph $T$ contains exactly one a line $\ell$ on scale $j$ (as $T \in \mathcal{S}_{1, j}^{\mathcal{R}}$ ), hence $n_{\ell}=j$ and $\left|g_{\ell}^{\left[n_{\ell}\right]}\right| \leq C_{1}^{-1} 2^{j+1}$, so that we can use that $2^{j} e^{-A_{3} 2^{j / \tau_{1}} / 4}$ is bounded by a constant. Moreover we have $e^{-A_{3} 2^{j / \tau_{1}} / 4} \leq e^{-\beta_{2} B_{2} 2^{n_{0} / \tau_{1}}} \leq\left|G_{j_{0}}\right|\left(2 \beta_{1} B_{1}\right)^{-1}|\varepsilon|^{j_{0}-1}$ if $\beta_{2}$ in (6.10) is chosen such that $\beta_{2} B_{2} \leq A_{3} / 4$ (see Remark 6.5). Therefore, we can conclude that if $j_{0} \geq 2$ the first sum in (7.16) can be bounded proportionally to $|\varepsilon||\varepsilon|^{j_{0}-1} e^{-A_{3} 2^{j / \tau_{1}} / 2}$. Hence the third bound in (7.11) follows for any value of $j_{0}$, with $D^{\prime}=A_{3} / 2$.

The second bound in (7.11) again can be proved by reasoning as in Ref. 13] for the contributions arising from self-energy graphs $T$ with $k_{T} \geq 2$. The contributions arising from self-energy graphs $T$ with with $k_{T}=1$ can be bounded as $|\varepsilon| \mathcal{Q} C_{1}^{-1} 2^{n+1} e^{-A_{3} 2^{n / \tau_{1}}} e^{-\kappa\left|\boldsymbol{\nu}_{v}\right| / 2}$ (as in the bound on the first sum 
in the r.h.s. of (7.16) ) because there is only one propagator on scale $n$. Then, if the derivative acts on the compact support function $\chi_{n_{0}}(|x|)$, one has that $2^{n_{0}} 2^{n+1} e^{-A_{3} 2^{n / \tau_{1}} / 2}$ is bounded by a constant for all $n>n_{0}$.

Remark 7.6 As suggested by the proof of Lemma 7.5 we shall fix $\beta_{2}$ in (6.10) such that one has $D^{\prime} \geq 2 \beta_{2} B_{2}$, where $D^{\prime}$ is the constant appearing in the last of 7.11). For future convenience we shall choose $\beta_{2}$ such that $D^{\prime}=4 \beta_{2} B_{2}$; see (8.21). We shall see below that it will be useful (even not necessary) also to choose $\beta_{1}$ in (6.10) such that $2 D \leq \beta_{1} B_{1}$.

Proposition 7.7 Assume that the set $\mathcal{E}^{[\infty]}$ has non-zero measure. Then for all $\varepsilon \in \mathcal{E}^{[\infty]}$ one has

$$
\left|g_{\ell}^{\left[n_{\ell}\right]}\right| \leq C_{1}^{-1} 2^{n_{\ell}+1}
$$

for all lines $\ell$ in any tree or self-energy graph. In particular the series (5.14) is uniformly convergent to a function analytic in $t$.

Proof. It follows from Lemma 7.1 by taking the limit $n \rightarrow \infty$ and using that the constant $c$ does not depend on $n$, that the bound (7.2) holds for all $j>n_{0}$. Then one can bound the product of propagators as done in the proof of Lemma [7.5] and using part of the decaying factors $e^{-\kappa\left|\boldsymbol{\nu}_{v}\right|}$ to obtain an overall factor $e^{-\kappa|\boldsymbol{\nu}| / 4}$ for any tree $\theta \in \Theta_{k, \boldsymbol{\nu}}$ contributing to $u_{\boldsymbol{\nu}}^{[k]}$.

\section{Measure of the set of admissible values}

To apply the above results we have still to construct the set $\mathcal{E}_{*}$ for which the Diophantine conditions (5.15) hold, and to show that such a set has positive measure. Here and henceforth we assume that the constants $n_{0}$ and $p$ are chosen according to Remark 6.6 and Remark 7.3] respectively.

Define recursively the sets $\mathcal{E}^{[n]}$ as follows. Set $\mathcal{E}^{\left[n_{0}\right]}=\mathcal{E}_{m}$ and, for $n \geq n_{0}+1$,

$$
\mathcal{E}^{[n]}:=\left\{\varepsilon \in \mathcal{E}^{[n-1]}:\left|i \boldsymbol{\omega} \cdot \boldsymbol{\nu}-\mathcal{M}^{[n-1]}(\boldsymbol{\omega} \cdot \boldsymbol{\nu} ; \varepsilon)\right|>C_{1}|\boldsymbol{\nu}|^{-\tau_{1}}\right\},
$$

for suitable Diophantine constants $C_{1}$ and $\tau_{1}$ (to be fixed later). It is clear that

$$
\mathcal{E}_{*} \cap \mathcal{E}_{m}=\mathcal{E}^{[\infty]}=\bigcap_{n=n_{0}}^{\infty} \mathcal{E}^{[n]}=\lim _{n \rightarrow \infty} \mathcal{E}^{[n]}
$$

Lemma 8.1 The functions $M^{[n]}(x ; \varepsilon)$ and their derivatives $\partial_{x} M^{[n]}(x ; \varepsilon)$ are $C^{1}$ extendible in the sense of Whitney outside $\mathcal{E}^{[n-1]}$, and for all $\varepsilon, \varepsilon^{\prime} \in \mathcal{E}^{[n-1]}$ one has

$$
\partial_{x}^{s} M^{[n]}\left(x ; \varepsilon^{\prime}\right)-\partial_{x}^{s} M^{[n]}(x ; \varepsilon)=\left(\varepsilon^{\prime}-\varepsilon\right) \partial_{\varepsilon} \partial_{x}^{s} M^{[n]}(x ; \varepsilon)+o\left(\varepsilon^{\prime}-\varepsilon\right),
$$

where $s=0,1$ and $\partial_{\varepsilon} \partial_{x}^{s} M^{[n]}(x ; \varepsilon)$ denotes the formal derivative with respect to $\varepsilon$ of $\partial_{x}^{s} M^{[n]}(x ; \varepsilon)$. Furthermore one has

$$
\left|\partial_{\varepsilon} \partial_{x} \mathcal{M}^{[n]}(x ; \varepsilon)\right| \leq D \sqrt{|\varepsilon|}, \quad\left|\partial_{\varepsilon} \partial_{x} M^{[n]}(x ; \varepsilon)\right| \leq D \sqrt{|\varepsilon|} e^{-D^{\prime} 2^{n / \tau_{1}}},
$$

for all $n>n_{0}$. One can take $D$ as in Lemma 7.5.

Proof. As the proof of Lemma 3 in Ref. [13. In order to obtain the inequality (8.4) one has to use the Remark [5.7] Of course, when expressing $\mathcal{M}^{[n]}(x ; \varepsilon)$ in terms of the self-energy values $\mathcal{V}_{T}(x ; \varepsilon)$ we have to bear in mind that the constant $2^{n_{0}}$ can be bounded in terms of $\varepsilon$, but it does not depend on $\varepsilon$ (as far as $\varepsilon$ varies in $\mathcal{E}_{m}$ and $n_{0}$ is chosen according to Remark (6.6), so that the derivatives with respect 
to $\varepsilon$ of $\mathcal{V}_{T}(x ; \varepsilon)$, as expressed in (5.9), act only on $\varepsilon^{k_{T}}$ and on the quantities $\mathcal{M}^{[j]}(x ; \varepsilon)$ appearing in the propagators. Hence $\partial_{\varepsilon} \mathcal{V}_{T}(x ; \varepsilon)$ and $\partial_{\varepsilon} \partial_{x} \mathcal{V}_{T}(x ; \varepsilon)$ can be studied as in Ref. 13. We simply note that when acting on some propagator $g_{\ell}^{\left[n_{\ell}\right]}$ the derivatives with respect to $\varepsilon$ can rise the power of the divisor $i x-\mathcal{M}^{\left[n_{\ell}-1\right]}(x ; \varepsilon)$, and if $n_{\ell}=n_{0}$ we have to use part of the exponential decay $e^{-A_{3} 2^{j / \tau_{1}}}$ (see (7.14) ) to take into account the extra factors $2^{n_{0}}$. The conclusion is that essentially the derivative with respect to $\varepsilon$ of $\mathcal{V}_{T}(x ; \varepsilon)$ admits the same bound (7.14) as $\mathcal{V}_{T}(x ; \varepsilon)$, possibly with different constants $A_{1}$ and $A_{2}$ (but still such that a bound like (7.15) is fulfilled, as far as their dependence on $\varepsilon$ is concerned), except that the exponent of $|\varepsilon|$ is $k-1$ instead of $k$.

Therefore for all $\varepsilon \in \mathcal{E}^{[n-1]}$ the quantities $\mathcal{M}^{[n]}(x ; \varepsilon)$ are well defined and formally differentiable (in the sense of Whitney) together with their derivatives with respect to $x$.

Lemma 8.2 There are two positive constants $\mathfrak{m}_{1}$ and $\mathfrak{m}_{2}$ such that

$$
\left|\partial_{\varepsilon} \mathcal{M}^{[n]}(x ; \varepsilon)\right| \geq \mathfrak{m}_{1}|\varepsilon|^{j_{0}-1}-\mathfrak{m}_{2} \sqrt{|\varepsilon|}|x|,
$$

for all $n \geq n_{0}$.

Proof. If we write

$$
\partial_{\varepsilon} \mathcal{M}^{[n]}(x ; \varepsilon)=\partial_{\varepsilon} \mathcal{M}^{[n]}(0 ; \varepsilon)+\int_{0}^{x} \mathrm{~d} x^{\prime} \partial_{\varepsilon} \partial_{x^{\prime}} \mathcal{M}^{[n]}\left(x^{\prime} ; \varepsilon\right),
$$

we have

$$
\left|\partial_{\varepsilon} \mathcal{M}^{[n]}(0 ; \varepsilon)\right| \geq\left|\partial_{\varepsilon} \mathcal{M}^{\left[n_{0}\right]}(0 ; \varepsilon)\right|-\sum_{j=n_{0}+1}^{n}\left|\partial_{\varepsilon}\left(\Xi_{j}(0 ; \varepsilon) M^{[j]}(0 ; \varepsilon)\right)\right|
$$

and we can bound

$$
\begin{aligned}
\left|\partial_{\varepsilon} \mathcal{M}^{\left[n_{0}\right]}(0 ; \varepsilon)\right| & \geq\left|\partial_{\varepsilon} \mathcal{M}_{j_{0}}^{\left[n_{0}\right]}(0 ; \varepsilon)\right|-\sum_{j=j_{0}+1}^{\infty} \sum_{T \in \mathcal{S}_{j, n_{0}}^{\mathcal{R}}}\left|\partial_{\varepsilon} \mathcal{V}_{T}(0 ; \varepsilon)\right| \\
& \geq \frac{j_{0}}{2}|\varepsilon|^{j_{0}-1}\left|G_{j_{0}}\right|+O\left(|\varepsilon|^{j_{0}-1} \sqrt{|\varepsilon|}\right) \geq \frac{j_{0}}{4}|\varepsilon|^{j_{0}-1}\left|G_{j_{0}}\right|,
\end{aligned}
$$

where we have reasoned as at the end of the proof of Lemma 8.1 in order to bound $\partial_{\varepsilon} \mathcal{V}_{T}(0 ; \varepsilon)$, and have used Lemma 6.4 and Remark 7.3 in order to fix $p$ in (7.8). Hence

$$
\begin{aligned}
\left|\partial_{\varepsilon} \mathcal{M}^{[n]}(0 ; \varepsilon)\right| & \geq \frac{j_{0}}{4}|\varepsilon|^{j_{0}-1}\left|G_{j_{0}}\right|+O\left(|\varepsilon|^{j_{0}-1} \sqrt{\varepsilon}\right) \\
& \geq \frac{j_{0}}{8}|\varepsilon|^{j_{0}-1} G_{j_{0}} \equiv \mathfrak{m}_{1}|\varepsilon|^{j_{0}-1},
\end{aligned}
$$

by the second inequality in (8.4) and by proceeding as at the end of the proof of Lemma 7.5 (see also Remark [7.6).

Furthermore one has

$$
\left|\int_{0}^{x} \mathrm{~d} x^{\prime} \partial_{\varepsilon} \partial_{x^{\prime}} \mathcal{M}^{[n]}\left(x^{\prime} ; \varepsilon\right)\right| \leq|x| \max _{x}\left|\partial_{\varepsilon} \partial_{x} \mathcal{M}^{[n]}(x ; \varepsilon)\right| \equiv \mathfrak{m}_{2} \sqrt{|\varepsilon|}|x|
$$

because of Lemma 8.1 and the assertion is proved.

Lemma 8.3 There are two positive constants $b$ and $\xi$ such that, for $\varepsilon_{0}$ small enough and $\varepsilon_{m}=2^{-m} \varepsilon_{0}$, one has

$$
\operatorname{meas}\left(\mathcal{E}^{[\infty]}\right)=\operatorname{meas}\left(\mathcal{E}_{m} \cap \mathcal{E}_{*}\right) \geq \frac{\varepsilon_{m}}{2}\left(1-b \varepsilon_{m}^{\xi}\right),
$$

where meas denotes the Lebesgue measure. The constants $b$ and $\xi$ are independent of $m$. 
Proof. Define $\mathcal{I}^{\left[n_{0}\right]}=\emptyset$ and $\mathcal{I}^{[n]}=\mathcal{E}^{[n-1]} \backslash \mathcal{E}^{[n]}$ for $n \geq n_{0}+1$; note that $\mathcal{I}:=\cup_{n=n_{0}}^{\infty} \mathcal{I}^{[n]}=\mathcal{E}_{m} \backslash \mathcal{E}^{[\infty]}$. Recall also that we have set $\mathcal{E}^{\left[n_{0}\right]}=\mathcal{E}_{m}$.

For all $n \geq n_{0}+1$ and for all $\boldsymbol{\nu} \in \mathbb{Z}_{*}^{d}$ define

$$
I^{[n]}(\boldsymbol{\nu})=\left\{\varepsilon \in \mathcal{E}^{[n-1]}:\left|i \boldsymbol{\omega} \cdot \boldsymbol{\nu}-\mathcal{M}^{[n-1]}(\boldsymbol{\omega} \cdot \boldsymbol{\nu} ; \varepsilon)\right| \leq C_{1}|\boldsymbol{\nu}|^{-\tau_{1}}\right\} .
$$

Each set $I^{[n]}(\boldsymbol{\nu})$ has "center" in a point $\varepsilon^{[n]}(\boldsymbol{\nu})$, defined implicitly by the equation

$$
i \boldsymbol{\omega} \cdot \boldsymbol{\nu}-\mathcal{M}^{[n]}\left(\boldsymbol{\omega} \cdot \boldsymbol{\nu} ; \varepsilon^{[n]}(\boldsymbol{\nu})\right)=0,
$$

where we are using the Whitney extension of $\mathcal{M}^{[n]}(\boldsymbol{\omega} \cdot \boldsymbol{\nu} ; \varepsilon)$ outside $\mathcal{E}^{[n-1]}$.

Therefore one has to exclude from the set $\mathcal{E}^{[n-1]}$ all the values $\varepsilon$ around $\varepsilon^{[n]}(\boldsymbol{\nu})$ in $I^{[n]}(\boldsymbol{\nu})$, and this has to be done for all $\boldsymbol{\nu} \in \mathbb{Z}_{*}^{d}$ satisfying

$$
|\boldsymbol{\omega} \cdot \boldsymbol{\nu}| \leq \frac{3}{2}\left|\mathcal{M}^{[n]}(\boldsymbol{\omega} \cdot \boldsymbol{\nu} ; \varepsilon)\right|,
$$

because otherwise one can bound $\left|i \boldsymbol{\omega} \cdot \boldsymbol{\nu}-\mathcal{M}^{[n]}(\boldsymbol{\omega} \cdot \boldsymbol{\nu} ; \varepsilon)\right| \geq|\boldsymbol{\omega} \cdot \boldsymbol{\nu}| / 3 \geq C_{1}|\boldsymbol{\nu}|^{-\tau_{1}}$ as soon as $\tau_{1} \geq \tau$ and $C_{1} \leq C_{0} / 3$.

For $\varepsilon$ small enough and for all $n \geq n_{0}$ one can bound

$$
\left|\mathcal{M}^{[n]}(0 ; \varepsilon)-\mathcal{M}^{\left[n_{0}\right]}(0 ; \varepsilon)\right| \leq 2 D|\varepsilon| e^{-D^{\prime} 2^{n_{0} / \tau_{1}}} \leq \beta_{1} B_{1}|\varepsilon| e^{-\beta_{2} B_{2} 2^{n_{0} / \tau_{1}}},
$$

by the third inequality in (7.11) of Lemma 7.5 applied repeatedly from scale $n_{0}+1$ to scale $n$, and having used that one has $\beta_{2} B_{2}<D^{\prime}$ and $2 D \leq \beta_{1} B_{1}$ (see Remark [7.6),

$$
\left|\mathcal{M}^{\left[n_{0}\right]}(0 ; \varepsilon)-\mathcal{M}_{j_{0}}^{\left[n_{0}\right]}(0 ; \varepsilon)\right|=O\left(|\varepsilon|^{j_{0}} \sqrt{|\varepsilon|}\right)
$$

if $p$ in (7.8) is chosen according to Remark 7.3 and

$$
\left|\mathcal{M}_{j_{0}}^{\left[n_{0}\right]}(0 ; \varepsilon)-\overline{\mathcal{M}}_{j_{0}}^{\left[n_{0}\right]}(0 ; \varepsilon)\right| \leq B_{1}|\varepsilon| e^{-B_{2} 2^{n_{0} / \tau_{1}}}
$$

by Lemma 6.3 so that one finds

$$
\left|\mathcal{M}^{[n]}(0 ; \varepsilon)-\overline{\mathcal{M}}_{j_{0}}^{\left[n_{0}\right]}(0 ; \varepsilon)\right|<2 G_{j_{0}}|\varepsilon|^{j_{0}},
$$

if $n_{0}$ is fixed as said in Remark [6.6] so that $2 \beta_{1} B_{1}|\varepsilon| e^{-\beta_{2} B_{2} 2^{n_{0} / \tau_{1}}} \leq|\varepsilon|^{j_{0}}\left|G_{j_{0}}\right|$.

Therefore one can bound

$$
\begin{aligned}
\left|\mathcal{M}^{[n]}(x ; \varepsilon)\right| & \leq\left|\mathcal{M}^{[n]}(0 ; \varepsilon)\right|+\left|\mathcal{M}^{[n]}(x ; \varepsilon)-\mathcal{M}^{[n]}(0 ; \varepsilon)\right| \\
& \leq\left|\overline{\mathcal{M}}_{j_{0}}^{\left[n_{0}\right]}(0 ; \varepsilon)\right|+2 G_{j_{0}}|\varepsilon|^{j_{0}}+D \sqrt{|\varepsilon|}|x| \\
& \leq 3 G_{j_{0}}|\varepsilon|^{j_{0}}+\frac{3}{2} D \sqrt{|\varepsilon|}\left|\mathcal{M}^{[n]}(x ; \varepsilon)\right|,
\end{aligned}
$$

with $x=\boldsymbol{\omega} \cdot \boldsymbol{\nu}$, for all $\boldsymbol{\nu}$ satisfying (8.11). We can conclude that there exists a constant $\mathfrak{D}$ such that one has

$$
\left|\mathcal{M}^{[n]}(\boldsymbol{\omega} \cdot \boldsymbol{\nu} ; \varepsilon)\right| \leq \varepsilon_{m}^{j_{0}} \mathfrak{D}
$$

for all $\boldsymbol{\nu}$ satisfying (8.11).

Hence we have to consider only the vectors $\boldsymbol{\nu} \in \mathbb{Z}_{*}^{d}$ satisfying not only (8.11) but also the inequality $|\boldsymbol{\omega} \cdot \boldsymbol{\nu}|<2 \varepsilon_{m}^{j_{0}} \mathfrak{D}$, i.e. for all $\boldsymbol{\nu} \in \mathbb{Z}_{*}^{d}$ such that

$$
|\boldsymbol{\nu}| \geq\left(\frac{C_{0}}{2 \varepsilon_{m}^{j_{0} \mathfrak{D}}}\right)^{1 / \tau}:=N_{0}
$$


We call $\mathcal{N}_{0}$ the set of $\boldsymbol{\nu} \in \mathbb{Z}_{*}^{d}$ which satisfy (8.11) and 8.13).

For such $\boldsymbol{\nu}$, by setting $x=\boldsymbol{\omega} \cdot \boldsymbol{\nu}$, one has

$$
\begin{aligned}
\left|\partial_{\varepsilon} \mathcal{M}^{[n]}(x ; \varepsilon)\right| & \geq \mathfrak{m}_{1}|\varepsilon|^{j_{0}-1}-\frac{3}{2} \mathfrak{m}_{2} \sqrt{|\varepsilon|}\left(2 \varepsilon_{m}^{j_{0}} \mathfrak{D}\right) \\
& \geq \frac{\mathfrak{m}_{1}}{2^{j_{0}-1}} \varepsilon_{m}^{j_{0}-1}\left(1-\frac{3 \mathfrak{m}_{2}}{2 \mathfrak{m}_{1}} \varepsilon_{m}^{3 / 2} 2^{j_{0}} \mathfrak{D}\right) \geq \frac{\mathfrak{m}_{1}}{2^{j_{0}}} \varepsilon_{m}^{j_{0}-1},
\end{aligned}
$$

so that the measure of the corresponding excluded set, which can be written as

$$
\int_{I^{[n]}(\boldsymbol{\nu})} \mathrm{d} \varepsilon=\int_{-1}^{1} \mathrm{~d} t \frac{\mathrm{d} \varepsilon(t)}{\mathrm{d} t}
$$

where $\varepsilon(t)$ is defined by

$$
i \boldsymbol{\omega} \cdot \boldsymbol{\nu}-\mathcal{M}^{[n]}(\boldsymbol{\omega} \cdot \boldsymbol{\nu} ; \varepsilon(t))=t \frac{C_{1}}{|\boldsymbol{\nu}|^{\tau_{1}}}
$$

will be bounded by

$$
\int_{I^{[n]}(\boldsymbol{\nu})} \mathrm{d} \varepsilon \leq \int_{-1}^{1} \mathrm{~d} t C_{1}|\boldsymbol{\nu}|^{-\tau_{1}} \frac{1}{\left|\partial_{\varepsilon} \mathcal{M}^{[n]}(\boldsymbol{\omega} \cdot \boldsymbol{\nu} ; \varepsilon(t))\right|} \leq \frac{2^{j_{0}+1}}{\mathfrak{m}_{1} \varepsilon_{m}^{j_{0}-1}} \frac{C_{1}}{|\boldsymbol{\nu}|^{\tau_{1}}},
$$

by (8.14).

This yields that we have to exclude from $\mathcal{E}^{[n-1]}$ a set

$$
\mathcal{I}^{[n]}=\bigcup_{\boldsymbol{\nu} \in \mathcal{N}_{0}} I^{[n]}(\boldsymbol{\nu})
$$

of measure bounded by

$$
\begin{aligned}
\operatorname{meas}\left(\mathcal{I}^{[n]}\right) & \leq \sum_{\boldsymbol{\nu} \in \mathcal{N}_{0}} \operatorname{meas}\left(I^{[n]}(\boldsymbol{\nu})\right) \leq \text { const. } \sum_{|\boldsymbol{\nu}| \geq N_{0}} \frac{C_{1}}{\varepsilon_{m}^{j_{0}-1}}|\boldsymbol{\nu}|^{-\tau_{1}} \\
& \leq \text { const. } \frac{C_{1}}{\varepsilon_{m}^{j_{0}-1}}\left(\frac{\varepsilon_{m}^{j_{0}}}{C_{1}}\right)^{\left(\tau_{1}-d\right) / \tau}=\text { const. } \varepsilon_{m}^{1+\xi^{\prime}},
\end{aligned}
$$

where $\xi^{\prime}=j_{0}\left(\tau_{1}-\tau-d\right) / \tau$, so that $\xi^{\prime}>0$ if $\tau_{1}>\tau+d$, which fixes the value of $\tau_{1}$.

We can easily prove that there exist two positive constants $E_{1}$ and $E_{2}$ such that one has

$$
\left|\varepsilon^{[n]}(\boldsymbol{\nu})-\varepsilon^{[n-1]}(\boldsymbol{\nu})\right| \leq \sqrt{\varepsilon_{m}} E_{1} e^{-E_{2} 2^{n / \tau_{1}}}
$$

for all $n \geq n_{0}+1$ and for all $\boldsymbol{\nu} \in \mathbb{Z}_{*}^{d}$. By setting $\delta \varepsilon=\varepsilon^{[n]}(\boldsymbol{\nu})-\varepsilon^{[n-1]}(\boldsymbol{\nu})$, we obtain (again by using Whitney extensions)

$$
\begin{aligned}
0= & i \boldsymbol{\omega} \cdot \boldsymbol{\nu}-\mathcal{M}^{[n]}\left(\boldsymbol{\omega} \cdot \boldsymbol{\nu} ; \varepsilon^{[n]}(\boldsymbol{\nu})\right) \\
= & i \boldsymbol{\omega} \cdot \boldsymbol{\nu}-\mathcal{M}^{[n-1]}\left(\boldsymbol{\omega} \cdot \boldsymbol{\nu} ; \varepsilon^{[n-1]}(\boldsymbol{\nu})+\delta \varepsilon\right) \\
& \quad-\mathcal{M}^{[n]}\left(\boldsymbol{\omega} \cdot \boldsymbol{\nu} ; \varepsilon^{[n]}(\boldsymbol{\nu})\right)+\mathcal{M}^{[n-1]}\left(\boldsymbol{\omega} \cdot \boldsymbol{\nu} ; \varepsilon^{[n]}(\boldsymbol{\nu})\right) \\
= & -\partial_{\varepsilon} \mathcal{M}^{[n-1]}\left(\boldsymbol{\omega} \cdot \boldsymbol{\nu} ; \varepsilon^{[n-1]}(\boldsymbol{\nu})\right) \delta \varepsilon+o(\delta \varepsilon) \\
& \quad-\left(\mathcal{M}^{[n]}\left(\boldsymbol{\omega} \cdot \boldsymbol{\nu} ; \varepsilon^{[n]}(\boldsymbol{\nu})\right)-\mathcal{M}^{[n-1]}\left(\boldsymbol{\omega} \cdot \boldsymbol{\nu} ; \varepsilon^{[n]}(\boldsymbol{\nu})\right)\right),
\end{aligned}
$$

by (8.3) in Lemma 8.1 hence one can use that

$$
\begin{aligned}
\mid \mathcal{M}^{[n]}(\boldsymbol{\omega} & \cdot \boldsymbol{\nu} ; \varepsilon)-\mathcal{M}^{[n-1]}(\boldsymbol{\omega} \cdot \boldsymbol{\nu} ; \varepsilon) \mid \\
& \leq D|\varepsilon| e^{-D^{\prime} 2^{n / \tau_{1}}} \\
& \leq \frac{D}{\beta_{1} B_{1}}|\varepsilon|\left(\beta_{1} B_{1} e^{-\beta_{2} B_{2} 2^{n / \tau_{1}}}\right) \leq B^{\prime}|\varepsilon|^{j_{0}} e^{-\beta_{2} B_{2} 2^{n / \tau_{1}}},
\end{aligned}
$$


with $B^{\prime}$ a suitable constant, by the third inequality of (7.11) in Lemma 7.5] by (6.10) and by Remark 7.6 Hence by (8.14) and (8.21) we obtain (8.20) with $E_{1}=4 B^{\prime} / \mathfrak{m}_{1}$ and $E_{2}=\beta_{2} B_{2}$.

For all $|\boldsymbol{\nu}| \geq \mathcal{N}_{0}$ fix $n_{*}=n_{*}(\boldsymbol{\nu})$ such that $\left|\varepsilon^{\left[n_{*}+1\right]}(\boldsymbol{\nu})-\varepsilon^{\left[n_{*}\right]}(\boldsymbol{\nu})\right| \leq C_{1}|\boldsymbol{\nu}|^{-\tau_{1}}$. One can choose $n_{*}(\boldsymbol{\nu}) \leq$ const. $\tau_{1} \log \log |\boldsymbol{\nu}|$.

Then for all $n_{0}+1 \leq n \leq n_{*}$ define $J^{[n]}(\boldsymbol{\nu})$ as

$$
J^{[n]}(\boldsymbol{\nu})=\left\{\varepsilon \in \mathcal{E}^{[n-1]}:\left|i \boldsymbol{\omega} \cdot \boldsymbol{\nu}-\mathcal{M}^{[n-1]}(\boldsymbol{\omega} \cdot \boldsymbol{\nu} ; \varepsilon)\right|<2 C_{1}|\boldsymbol{\nu}|^{-\tau_{1}}\right\} ;
$$

by construction all the sets $I^{[n]}(\boldsymbol{\nu})$ fall inside $J^{\left[n_{*}\right]}(\boldsymbol{\nu})$ as soon as $n>n_{*}$. Then we can bound meas $(\mathcal{I})$ by the sum of the measures of the sets $J^{\left[n_{0}+1\right]}(\boldsymbol{\nu}), \ldots, J^{\left[n_{*}\right]}(\boldsymbol{\nu})$ for all $\boldsymbol{\nu} \in \mathbb{Z}_{*}^{d}$ such that $|\boldsymbol{\nu}| \geq \mathcal{N}_{0}$. Such a measure will be bounded by

$$
\text { const. } \sum_{|\boldsymbol{\nu}| \geq \mathcal{N}_{0}} n_{*}(\boldsymbol{\nu}) \frac{C_{1}}{\varepsilon_{m}^{j_{0}-1}}|\boldsymbol{\nu}|^{-\tau_{1}} \leq \text { const. } \varepsilon_{m}^{1+\xi},
$$

with a value $\xi$ smaller than $\xi^{\prime}$ in order to take into account the logarithmic corrections due to the factor $n_{*}(\boldsymbol{\nu})$.

Proposition 8.4 Define the set of admissible values of $\mathcal{E}_{*}$ as in Definition $\left[5.10\right.$ with $C_{1}=C_{0} / 3$ and $\tau_{1}>\tau+d$. Then one has

$$
\lim _{m \rightarrow \infty} \frac{\operatorname{meas}\left(\mathcal{E}_{m} \cap \mathcal{E}_{*}\right)}{\operatorname{meas}\left(\mathcal{E}_{m}\right)}=1
$$

Proof. It is an immediate consequence of the definitions and of Lemma 8.2

\section{Properties of the renormalized expansion}

To complete the proof of existence of a quasi-periodic solution of (2.9) we have to show that the function defined by the renormalized expansion (5.14) solves the equation (2.9). Set $\mathcal{E}_{+}=\cup_{m=0}^{\infty} \mathcal{E}_{m} \cap \mathcal{E}_{*}$ : such a set contains the admissible values of $\varepsilon$ in $\left[0, \varepsilon_{0}\right]$. Define analogously $\mathcal{E}_{-}$for the interval $\left[-\varepsilon_{0}, 0\right]$, and set $\mathcal{E}=\mathcal{E}_{+} \cup \mathcal{E}_{-}$.

Lemma 9.1 For all $\varepsilon \in \mathcal{E}$ the function $\bar{u}(t)$ defined through (5.14) solves the equation

$$
\bar{u}=g\left(R+\varepsilon Q \bar{u}^{2}\right),
$$

where $g$ is the pseudo-differential operator with kernel $g(\boldsymbol{\omega} \cdot \boldsymbol{\nu})=1 / i \boldsymbol{\omega} \cdot \boldsymbol{\nu}$.

Proof. As in Section 8 of Ref. [13].

So far we proved that there exists a function $\bar{u}(t)=U(\boldsymbol{\omega} t ; \varepsilon)$ which solves (2.9) for $\varepsilon$ in a suitable large measure Cantor set $\mathcal{E}$. For $g$ given by $g(t)=i \varepsilon Q(t) \bar{u}(t)$, Proposition 2.3 proves that $\phi(t)$ given in (2.6) solves (1.1) and is quasi-periodic.

In principle, if we set $\Omega_{\varepsilon}=\Omega_{0}+\langle g\rangle$, $\phi$ could be of the form

$$
\phi(t)=\Phi\left(\underline{\omega}_{1} t, \omega_{0} t, \Omega_{0} t, \Omega_{\varepsilon} t\right) \equiv e^{i \Omega_{\varepsilon} t} \tilde{\Phi}\left(\underline{\omega}_{1} t, \omega_{0} t, \Omega_{0} t\right),
$$

as it depends on $\bar{u}(t)$, and an extra frequency arises from the integral of the average of $g_{0}+g$ in the definition of $\Phi(t)$. But this is not the case, because the function $\tilde{\Phi}$ is of the form $\tilde{\Phi}=\left(\underline{\omega}_{1} t, \omega_{0} t\right)$, that is its dependence on $t$ is only through the variables $\omega_{0} t$ and $\underline{\omega}_{1} t$. This follows from the following property. 
Lemma 9.2 Let $\bar{u}$ be the function defined through the renormalized expansion 5.14): then $u_{\boldsymbol{\nu}}^{[k]} \neq 0$ requires that in $\boldsymbol{\nu}=\left(\underline{m}, n_{1}, n_{2}\right)$ one has $n_{2}=2$.

Proof. The proof is by induction on $k$. For $k=0$ the result is obvious from the relation $(i \boldsymbol{\omega} \cdot \boldsymbol{\nu}) u_{\boldsymbol{\nu}}^{(0)}=R_{\boldsymbol{\nu}}$ in (2.16) and from the identity $R_{\boldsymbol{\nu}}=P_{\underline{m}}^{(1)} \mathcal{F}_{n_{1}}^{(2)} \delta_{n_{2}, 2}$. Let us assume that $u_{\boldsymbol{\nu}}^{\left[k^{\prime}\right]} \propto \delta_{n_{2}, 2}$ for all $k^{\prime}<k$. Then to order $k$ the second relation in (2.16) yields that one can have $u_{\boldsymbol{\nu}}^{[k]} \neq 0$ only if $\boldsymbol{\nu}=\boldsymbol{\nu}_{0}+\boldsymbol{\nu}_{1}+\boldsymbol{\nu}_{2}$ : for the last component $n_{2}$ of the vector $\boldsymbol{\nu}$ the identity $Q_{\boldsymbol{\nu}}=\delta_{\underline{m}, \underline{0}} \mathcal{F}_{n_{1}}^{(-2)} \delta_{n_{2},-2}$ and the inductive assumption give $n_{2}=-2+2+2=2$.

Hence $\bar{u}(t)=e^{2 i \Omega_{0} t} \tilde{U}\left(\underline{\omega}_{1} t, \omega_{0} t\right)$, with $\tilde{U}$ analytic and periodic in its arguments. By taking into account that one has $Q(t)=e^{-2 i \Omega_{0} t-2 i \psi_{0}(t)}$, with $\psi_{0}(t)$ depending on $t$ only through the variable $\omega_{0} t$, one has $Q(t) \bar{u}(t)=e^{-2 i \psi_{0}(t)} \tilde{U}\left(\underline{\omega}_{1} t, \omega_{0} t\right)$. As a consequence $\phi(t)$ is a quasi-periodic function with $d$ fundamental frequencies $\underline{\omega}_{1}, \omega_{0}, \Omega_{\varepsilon}$, and the dependence on the last frequency is only through the factor $e^{i \Omega_{\varepsilon} t}$, exactly as in the unperturbed case (2.3). As anticipated in Remark 2.2 the same result can be obtained by starting from the unperturbed solution given by the second function in (2.4), and an analogous result is found, so that we can conclude that the system is reducible for $\varepsilon \in \mathcal{E}$.

So the solution $\bar{u}(t)$ describes the motion on a $d$-dimensional maximal torus which is the continuation in $\varepsilon$ of an unperturbed $d$-dimensional torus. The rotation vector of the latter is $\boldsymbol{\omega}=\left(\underline{\omega}_{1}, \omega_{0}, \Omega_{0}\right)$, while, as an effect of the perturbation, only the last component of the rotation vector is changed into a new frequency $\Omega_{\varepsilon}=\Omega_{0}+\langle g\rangle$ : this provides a simple physical interpretation of the the quantity $\langle g\rangle$. It is likely that the new frequency $\Omega_{\varepsilon}$ is such that the vector $\left(\underline{\omega}_{1}, \omega_{0}, \Omega_{\varepsilon}\right)$ is still Diophantine. This does not follow directly from our analysis, but we expect that this is the case.

\section{Null renormalization}

We are left with the case in which one has $G_{j}=0$ for all $j \in \mathbb{N}$. In such a case we need no resummations, as it will become clear from the analysis. Hence we use the simpler multiscale decomposition of the propagators given by (5.1), with $C_{1}=C_{0}$. The following result holds.

Lemma 10.1 One has $\psi_{n-1}(x) \psi_{n}(x)=\psi_{n-1}(x)$ and

$$
\psi_{0}(x)+\sum_{j=1}^{n} \chi_{j-1}(x) \psi_{j}(x)=\psi_{n}(x),
$$

for all $n \in \mathbb{N}$ and for all $x \in \mathbb{R}$.

Proof. Both relations follow immediately from the definitions.

Then we consider the same tree expansion leading to (5.3), where no resummation is performed. The following result allows us to get rid of some trees.

Lemma 10.2 Suppose that one has $G_{j}=0$ for all $j \in \mathbb{N}$. Then in the tree expansion of $u_{\nu}^{(k)}$ in (5.3) the sum over $\Theta_{k, \nu}$ can be restricted only to trees which do not contain any vertex $v$ such that one of the entering lines carries the same momentum of the exiting line.

Proof. If there were no the scale labels this would follow from item (a) in Proposition 3.15. The presence of the scales could destroy in principle the compensation mechanism responsible of the cancellation among the values of the various trees. But it is sufficient to note that the coefficient $u_{\nu}^{(k)}$ is obtained by summing over all the possible scale labels, and in this way we reconstruct for each line $\ell$ the original propagator $1 / i \boldsymbol{\omega} \cdot \boldsymbol{\nu}_{\ell}$ (just use (10.1) for $n \rightarrow \infty$ ), hence we can apply the cited result. 
Remark 10.3 If $G_{j}=0$ for all $j \in \mathbb{N}$ formal solubility of the equation (1.4) requires no condition on the coefficients $\alpha^{(k)}$, which therefore can be arbitrarily fixed (cf. Lemma [3.5). For simplicity we can still fix $\alpha^{(k)}=0$ for all $k$, even if this not strictly necessary. Of course one can ask what happens for other choices of the coefficients $\alpha^{(k)}$, but we do not investigate further such a problem because the case in which all $G_{j}$ are vanishing is rather special, and likely it can really arise only in trivial situations (like $p_{1} \equiv 0$ ).

We define the clusters according to the definition previously done, whereas we slightly change the definition of self-energy graph, to make it more suitable for our purposes in the present case (cf. Ref. [2]). An important feature is that the propagators are not changed by any resummation procedure, so that for any line $\ell$ the (two) scales for which the corresponding propagator is not vanishing are uniquely fixed by $\boldsymbol{\nu}_{\ell}$.

Definition 10.4 (Self-Energy Graph) We call self-energy graph any cluster $T$ of a tree $\theta$ which satisfies

1. $T$ has only one entering line $\ell_{T}^{\text {in }}$ and only one exiting line $\ell_{T}^{\text {out }}$;

2. The momentum of $T$ is zero, i.e. $\boldsymbol{\nu}_{T}=\sum_{v \in B(T)} \boldsymbol{\nu}_{v}=\mathbf{0}$.

3. The mode labels $\boldsymbol{\nu}_{v}, v \in B(T)$, satisfy the relation $\sum_{v \in B(T)}\left|\boldsymbol{\nu}_{v}\right|<2^{\left(n_{\text {ext }}-4\right) / \tau}$, where $n_{\text {ext }}$ is the minimum between the scales of the external lines of $T$.

We call self-energy line any line $\ell_{T}^{\text {out }}$ which exits from a self-energy graph $T$. We call normal line any line which is not a self-energy line.

The self-energy value is then defined as before (see [5.9), with the only difference that now the propagators are $g_{\ell}^{\left(n_{\ell}\right)}$ (because they are not renormalized).

The aim of the last item in the definition of self-energy graph is that, given a self-energy graph, if we sum over all the scales of the internal lines compatible with the cluster structure, which yields that for each line $\ell \in L(T)$ one has $n_{\ell}<n_{\text {ext }}$, if $n_{\text {ext }}=\min \left\{n_{\ell_{T}^{\text {out }}}, n_{\ell_{T}^{\text {in }}}\right\}$, then we reconstruct for each line $\ell$ a propagator $\psi_{n_{\mathrm{ext}}+1}\left(\boldsymbol{\omega} \cdot \boldsymbol{\nu}_{\ell}\right) / i \boldsymbol{\omega} \cdot \boldsymbol{\nu}_{\ell}$, with $\psi_{n_{\mathrm{ext}}+1}\left(\boldsymbol{\omega} \cdot \boldsymbol{\nu}_{\ell}\right)=1$. The last assertion is implied from the following result.

Lemma 10.5 For any self-energy graph $T$, by setting $n_{\text {ext }}=\min \left\{n_{\ell_{T}^{\text {out }}}, n_{\ell_{T}^{\text {in }}}\right\}$, one can have $\mathcal{V}_{T}(\boldsymbol{\omega} \cdot \boldsymbol{\nu}) \neq$ 0 only if $n_{\ell} \leq n_{\mathrm{ext}}-2$ for any line $\ell \in L(T)$.

Proof. By definition of scales one has $C_{0} 2^{-n_{\text {ext }}-1} \leq|\boldsymbol{\omega} \cdot \boldsymbol{\nu}| \leq C_{0} 2^{-n_{\text {ext }}+1}$. The third item in the definition of self-energy graph gives $\left|\boldsymbol{\omega} \cdot \boldsymbol{\nu}_{\ell}^{0}\right|>C_{0} 2^{-\left(n_{\text {ext }}-4\right) / \tau}$ (see (15.10) for the definition of $\boldsymbol{\nu}_{\ell}^{0}$ ), hence by the Diophantine condition (2.14) on $\boldsymbol{\omega}$ one obtains

$$
\left|\boldsymbol{\omega} \cdot \boldsymbol{\nu}_{\ell}\right| \geq\left|\boldsymbol{\omega} \cdot \boldsymbol{\nu}_{\ell}^{0}\right|-|\boldsymbol{\omega} \cdot \boldsymbol{\nu}| \geq C_{0} 2^{-\left(n_{\mathrm{ext}}-4\right)}-C_{0} 2^{-\left(n_{\mathrm{ext}}-1\right)} \geq C_{0} 2^{-\left(n_{\mathrm{ext}}-3\right)},
$$

so that $\chi_{n^{\prime}-1}\left(\boldsymbol{\omega} \cdot \boldsymbol{\nu}_{\ell}\right)=0$ for $n^{\prime}>n_{\mathrm{ext}}-2$.

Definition 10.6 (Localization) For any self-energy graph $T$ we can define the localized part of the self-energy value $\mathcal{V}_{T}(\boldsymbol{\omega} \cdot \boldsymbol{\nu})$ as

$$
\mathcal{L} \mathcal{V}_{T}(\boldsymbol{\omega} \cdot \boldsymbol{\nu})=\mathcal{V}_{T}(0)
$$

and the regularized part as

$$
\mathcal{R} \mathcal{V}_{T}(\boldsymbol{\omega} \cdot \boldsymbol{\nu})=(\boldsymbol{\omega} \cdot \boldsymbol{\nu}) \int_{0}^{1} \mathrm{~d} t \partial \mathcal{V}_{T}(t \boldsymbol{\omega} \cdot \boldsymbol{\nu})
$$

where $\partial$ denotes derivative with respect to the argument, so that $\partial \mathcal{V}_{T}(t \boldsymbol{\omega} \cdot \boldsymbol{\nu})=\partial \mathcal{V}_{T}(x) /\left.\partial x\right|_{x=t \boldsymbol{\omega} \cdot \boldsymbol{\nu}}$. We shall call $\mathcal{L}$ and $\mathcal{R}$ the localization and regularization operator, respectively. 
By definition of self-energy value, one has

$$
\partial \mathcal{V}_{T}(t \boldsymbol{\omega} \cdot \boldsymbol{\nu})=\varepsilon^{k_{T}}\left(\prod_{v \in B(T)} F_{v}\right) \sum_{\ell \in L(T)} \partial g^{\left(n_{\ell}\right)}\left(\boldsymbol{\omega} \cdot \boldsymbol{\nu}_{\ell}(t)\right)\left(\prod_{\ell^{\prime} \in L(T) \backslash \ell} g^{\left(n_{\ell^{\prime}}\right)}\left(\boldsymbol{\omega} \cdot \boldsymbol{\nu}_{\ell^{\prime}}(t)\right)\right)
$$

where $\boldsymbol{\nu}_{\ell}(t)=\boldsymbol{\nu}_{\ell}^{0}$ if $\ell$ is not along the path connecting the external lines of $T$, and $\boldsymbol{\nu}_{\ell}(t)=\boldsymbol{\nu}_{\ell}^{0}+t \boldsymbol{\nu}$ otherwise.

The definition above suggests a further splitting of the tree values To each self-energy graph $T$ we associate a localization label which can be either $\mathcal{L}$ or $\mathcal{R}$ : the first one means that we have to compute the self-energy value for $\boldsymbol{\omega} \cdot \boldsymbol{\nu}=0$, while the second one tells us that we have to replace $\mathcal{V}_{T}(\boldsymbol{\omega} \cdot \boldsymbol{\nu})$ with $\mathcal{R} \mathcal{V}_{T}(\boldsymbol{\omega} \cdot \boldsymbol{\nu})$ as given by (10.3). Since a self-energy graph can contain other self-energy graphs, the application of the localization and regularization operators has to be performed iteratively by starting from the outermost (or maximal) self-energy graphs to end up with the innermost ones.

Lemma 10.7 Suppose that one has $G_{j}=0$ for all $j \in \mathbb{N}$. Then in the tree expansion of $u_{\nu}^{(k)}$ in (5.3) only trees with localization label $\mathcal{R}$ have to been retained.

Proof. Given a maximal self-energy graph $T$ consider the localized part of its self-energy graph. First of all note that the entering line of $T$ cannot enters the same vertex $v$ which the exiting line of $T$ comes out from (as a consequence of Lemma 10.2). For the remaining trees we can sum over all the scale labels compatible with the cluster structure, and apply Lemma 10.5 (which allows us to replace the support compact functions with 1). Then we can apply the cancellation mechanism leading to Lemma 3.12 indeed one immediately realizes that the cancellation works for fixed mode labels (see Remark 3.11).

Then $\mathcal{V}_{T}(0)=0$, so that we can replace $\mathcal{V}_{T}(\boldsymbol{\omega} \cdot \boldsymbol{\nu})$ with $\mathcal{R} \mathcal{V}_{T}(\boldsymbol{\omega} \cdot \boldsymbol{\nu})$, as given by (10.4). Here $\boldsymbol{\nu}$ is the momentum of the line entering $T$.

Next look at a self-energy graph $T^{\prime}$ contained inside $T$ and which is maximal (that is the only selfenergy graph containing $T^{\prime}$ is $T$ itself), and suppose we are considering a contribution to $\mathcal{R} \mathcal{V}_{T}(\boldsymbol{\omega} \cdot \boldsymbol{\nu})$ in which the derivative acts on some propagator external to $T^{\prime}$. The momentum $\boldsymbol{\nu}^{\prime}(t)$ flowing through the entering line $\ell_{T^{\prime}}^{\text {in }}$ of $T^{\prime}$ is either $\boldsymbol{\nu}^{\prime}(t)=\boldsymbol{\nu}_{\ell_{T^{\prime}}^{\text {in }}}^{0}$ or $\boldsymbol{\nu}^{\prime}(t)=\boldsymbol{\nu}_{\ell_{T^{\prime}}^{\text {in }}}^{0}+t \boldsymbol{\nu}$, so that for each line $\ell^{\prime} \in L\left(T^{\prime}\right)$ one has either $\boldsymbol{\nu}_{\ell^{\prime}}=\boldsymbol{\nu}_{\ell^{\prime}}^{0}$ or $\boldsymbol{\nu}_{\ell^{\prime}}=\boldsymbol{\nu}_{\ell^{\prime}}^{0}+\boldsymbol{\nu}^{\prime}(t)$. So when we compute the localized part of the self-energy value of $T^{\prime}$, we have to put $\boldsymbol{\nu}^{\prime}(t)=0$, and we can reason exactly as before for $T$ : then the same cancellation mechanism applies.

If instead the derivative in (10.4) acts on the self-energy value $\mathcal{V}_{T}\left(\boldsymbol{\omega} \cdot \boldsymbol{\nu}^{\prime}(t)\right)$ than we can write $\mathcal{V}_{T}\left(\boldsymbol{\omega} \cdot \boldsymbol{\nu}^{\prime}(t)\right)=\mathcal{L} \mathcal{V}_{T}\left(\boldsymbol{\omega} \cdot \boldsymbol{\nu}^{\prime}(t)\right)+\mathcal{R} \mathcal{V}_{T}\left(\boldsymbol{\omega} \cdot \boldsymbol{\nu}^{\prime}(t)\right)$, and of course the first term gives no contribution as it is a constant. Hence also in such a case we can get rid of the localized part of the self-energy value.

We can iterate the argument until no further self-energy graph is left, and the assertion follows.

Hence we have to consider the tree expansion (5.3), and retain only self-energy clusters with localization label $\mathcal{R}$. The discussion then becomes standard (see for instance Ref. 15), and for each self-energy graph $T$, if $\boldsymbol{\nu}$ is the momentum flowing through its external lines, we obtain a gain factor $\boldsymbol{\omega} \cdot \boldsymbol{\nu}$, which compensate exactly one of the propagators of the external lines of $T$, say that of the exiting line (self-energy line). Of course one has to control that no line is differentiated more than once, but this is a standard argument (again we refer to Ref. 15] for details). At the end we obtain that $\operatorname{Val}(\theta)$ admits a bound like (5.6), with the only difference that the propagators can be differentiated so that they have to be bounded as they were quadratic and not linear. On the other hand only normal lines have to be considered, as the self-energy lines are compensated by the mechanism described above, and they are bounded through (5.5). And the bound (5.5) still holds with the new definition of self-energy graph, as shown in Ref. [15].

The conclusion is that the series defining $u(t)$ is convergent, and it turns out to be analytic in $\varepsilon$. In particular this means that no value of $\varepsilon$ has to be discarded in such a case. Moreover $\langle g\rangle=0$, because 
$\langle g\rangle=i \varepsilon\langle Q u\rangle$, and $\langle Q u\rangle=0$ by item (a) in Proposition 3.15 and the hypothesis that one has $G_{j}=0$ for all $j \in \mathbb{N}$. In particular one has $\Omega_{\varepsilon}=\Omega_{0}$.

Therefore the case in which $G_{j}=0$ for all $j$ corresponds to have an integrable system. Note that the condition $G_{j}=0$ for all $j \in \mathbb{N}$ is a condition on the perturbation itself, so that it is not something that has to be checked while carrying on any iterative scheme to solve the problem.

\section{References}

[1] J. C. A. Barata, On Formal Quasi-Periodic Solutions of the Schrödinger Equation for a Two-Level System with a Hamiltonian Depending Quasi-Periodically on Time, Rev. Math. Phys. 12 (2000), no. $1,25-64$.

[2] M. V. Bartuccelli, G. Gentile, Lindstedt series for perturbations of isochronous systems: a review of the general theory, Rev. Math. Phys. 14 (2002), no. 2, 121-171.

[3] H. Bohr, Ueber fastperiodische ebene Bewegungen, Comment. Math. Helv. 4 (1932), 51-64. H. Bohr, Kleinere Beiträge zur Theorie der Fastperiodischen Funktionen, Danske Vid. Selsk. Mat.Fys. Medd. 10 (1930), no. 10, 5-11.

[4] H. Broer, J. Puig, C. Simó, Resonance tongues and instability pockets in the quasi-periodic HillSchrödinger equation, Comm. Math. Phys. 241 (2003), no. 2-3, 467-503.

[5] H. Broer, C. Simó, Hill's equation with quasi-periodic forcing: resonance tongues, instability pockets and global phenomena, Bol. Soc. Bras. Mat. 29 (1998), no. 2, 253-293.

[6] Ch.-Q. Cheng, Birkhoff-Kolmogorov-Arnold-Moser tori in convex Hamiltonian systems, Comm. Math. Phys. 177 (1996), no. 3, 529-559.

[7] Ch.-Q. Cheng, Lower-dimensional invariant tori in the regions of instability for nearly integrable Hamiltonian systems, Comm. Math. Phys. 203 (1999), no. 2, 385-419.

[8] L. Chierchia, Quasi-periodic Schrödinger operators in one dimension, absolutely continuous spectra, Bloch waves and integrable Hamiltonian systems, Quaderni del Consiglio Nazionale delle Ricerche, Firenze, 1986.

[9] L. Chierchia, Absolutely continuous spectra of quasi-periodic Schrödinger operators, J. Math. Phys. 28 (1987), no. 12, 2891-2898.

[10] E. I. Dinaburg, Ja. G. Sinaŭ, The one-dimensional Schrödinger equation with quasiperiodic potential, Funkcional. Anal. i Priložen. 9 (1975), no. 4, 8-21.

[11] L. H. Eliasson, Floquet solutions for the 1-dimensional quasi-periodic Schrödinger equation, Comm. Math. Phys. 146 (1992), no. 3, 447-482.

[12] G. Gallavotti, G. Gentile, Hyperbolic low-dimensional invariant tori and summations of divergent series, Comm. Math. Phys. 227 (2002), no. 3, 421-460.

[13] G. Gentile, Quasi-periodic solutions for two-level systems, Comm. Math. Phys. 242 (2002), no. 1-2, 221-250.

[14] G. Gentile, G. Gallavotti, Degenerate elliptic tori, Preprint, 2004.

[15] G. Gentile, V. Mastropietro, Methods for the analysis of the Lindstedt series for KAM tori and renormalizability in classical mechanics. A review with some applications, Rev. Math. Phys. 8 (1996), no. 3, 393-444. 
[16] M. Herman, Some open problems in dynamical systems, Proceedings of the International Congress of Mathematicians, Vol. II (Berlin, 1998), Doc. Math. 1998, Extra Vol. II, 797-808.

[17] D.B. Hinton, J.K. Shaw, On the absolutely continuous spectrum of the perturbed Hill's equation, Proc. London Math. Soc. (3) 50 (1985), no. 1, 175-192.

[18] R. Johnson, J. Moser, The rotation number for almost periodic potentials, Commun. Math. Phys. 84 (1982), no. 3, 403-438.

[19] Y. Katznelson, An Introduction to harmonic analysis, Dover, New York, 1978.

[20] R. Krikorian, Réductibilité presque partout des flots fibrés quasi-périodiques à valeurs dans des groupes compacts, Ann. Sci. École Norm. Sup. (4) 32 (1999), no. 2, 187-240.

[21] R. Krikorian, Global density of reducible quasi-periodic cocycles on $T^{1} \times \mathrm{SU}(2)$, Ann. of Math. (2) 154 (2001), no. 2, 269-326.

[22] W. Magnus, S. Winkler, Hill's Equation, Dover, New York, 1979.

[23] J. Moser, J. Pöschel, An extension of a result by Dinaburg and Sinaı̆ on quasiperiodic potentials, Comment. Math. Helv. 59 (1984), no. 1, 39-85.

[24] F.S. Rofe-Beketov, A finiteness test for the number of discrete levels which can be introduced into the gaps of the continuous spectrum by perturbations of a periodic potential (Russian), Dokl. Akad. Nauk SSSR 156 (1964), 515-518.

[25] H. Rüssmann, On the one-dimensional Schrödinger equation with a quasiperiodic potential, Nonlinear dynamics (Internat. Conf., New York, 1979), Ann. New York Acad. Sci., 357, New York Acad. Sci., New York, 1980.

[26] E. Sorets, T. Spencer, Positive Lyapunov exponents for Schrödinger operators with quasi-periodic potentials, Commun. Math. Phys. 142 (1991), no. 3, 543-566.

[27] V.A. Želudev, The eigenvalues of a perturbed Schrödinger operator with periodic potential (Russian), Problems of Mathematical Physics, no. 2, Spectral Theory, Diffraction Problems, pp. 108123, Izdat. Leningrad. Univ., Leningrad, 1967.

[28] V.A. Želudev, The perturbation of the spectrum of the one-dimensional selfadjoint Schrödinger operator with periodic potential (Russian), Problems of Mathematical Physics, no. 4, Spectral Theory. Wave Processes, pp. 61-82, Izdat. Leningrad. Univ., Leningrad, 1970. 